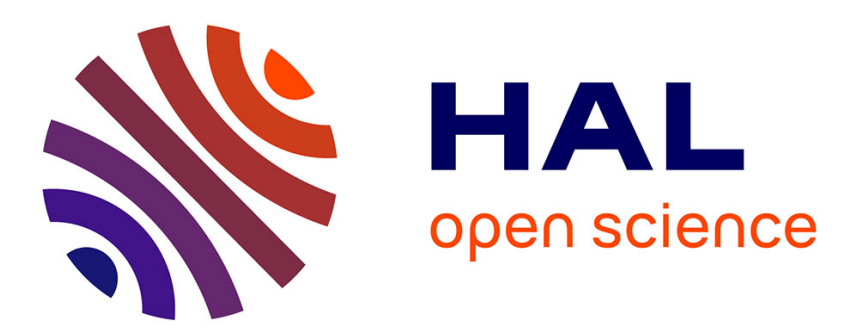

\title{
Stable perfectly matched layers for a class of anisotropic dispersive models. Part I: Necessary and sufficient conditions of stability
}

Eliane Bécache, Maryna Kachanovska

\section{- To cite this version:}

Eliane Bécache, Maryna Kachanovska. Stable perfectly matched layers for a class of anisotropic dispersive models. Part I: Necessary and sufficient conditions of stability. ESAIM: Mathematical Modelling and Numerical Analysis, 2017, 51 (6), pp.2399-2434. 10.1051/m2an/2017019 . hal-01356811v2

\section{HAL Id: hal-01356811 \\ https://hal.science/hal-01356811v2}

Submitted on 10 May 2021

HAL is a multi-disciplinary open access archive for the deposit and dissemination of scientific research documents, whether they are published or not. The documents may come from teaching and research institutions in France or abroad, or from public or private research centers.
L'archive ouverte pluridisciplinaire HAL, est destinée au dépôt et à la diffusion de documents scientifiques de niveau recherche, publiés ou non, émanant des établissements d'enseignement et de recherche français ou étrangers, des laboratoires publics ou privés. 


\title{
STABLE PERFECTLY MATCHED LAYERS FOR A CLASS OF ANISOTROPIC DISPERSIVE MODELS. PART I: NECESSARY AND SUFFICIENT CONDITIONS OF STABILITY. EXTENDED VERSION*
}

\author{
Éliane BÉCAChe ${ }^{1,2}$ AND Maryna KaChanovska ${ }^{1,3}$
}

\begin{abstract}
In this work we consider a problem of modelling of $2 \mathrm{D}$ anisotropic dispersive wave propagation in unbounded domains with the help of perfectly matched layers (PML). We study the Maxwell equations in passive media with a frequency-dependent diagonal tensor of dielectric permittivity and magnetic permeability. An application of the traditional PMLs to this kind of problems often results in instabilities. We provide a recipe for the construction of new, stable PMLs. For a particular case of non-dissipative materials, we show that a known necessary stability condition of the perfectly matched layers is also sufficient. We illustrate our statements with theoretical and numerical arguments.
\end{abstract}

1991 Mathematics Subject Classification. 65M12, 35Q60.

\section{INTRODUCTION}

The problem of wave propagation in anisotropic dispersive media, e.g. in negative index metamaterials or plasmas, is both of theoretical and practical interest. For some applications it is necessary to model the time-domain wave propagation in unbounded (or semi-bounded) domains. One of the ways to bound the computational domain is offered by the use of the perfectly matched layers (PMLs), a method introduced by Bérenger in $[1,2]$. For an application of this technique to some dispersive materials, for example, plasmas, see $[3-7]$, and for the use of the method in isotropic metamaterials see the papers by Cummer [8] and Bécache et al. [9]. However, it is well known that the PMLs often exhibits instabilities in the presence of dispersion and/or anisotropy $[9,10]$. Multiple attempts were made to overcome this problem [11], but, to our knowledge, no recipe to construct stable PMLs for an arbitrary hyperbolic system exists.

Indeed, there are other ways to tackle the problem of the unboundedness of the domain; a non-exhaustive list of those includes the FEM/BEM coupling [12,13], methods based on pole condition $[14,15]$ or various absorbing boundary conditions [16-20].

Keywords and phrases: perfectly matched layers, stability, Maxwell equations, passive metamaterials, Laplace transform

* This work was supported by a public grant as part of the Investissement d'avenir project, reference ANR-11-LABX-0056-LMH, LabEx LMH, as well as a co-financing program PRESTIGE.

1 Laboratoire Poems (UMR 7231 CNRS/Inria/ENSTA ParisTech), ENSTA ParisTech, 828 Boulevard des Maréchaux 91120 Palaiseau, France

2 E-mail: eliane.becache@inria.fr

3 E-mail: maryna.kachanovska@ensta.fr 
We concentrate on the construction of stable in the time domain PMLs for a class of anisotropic dispersive models that can be described in the frequency domain as a wave equation with frequency-dependent coefficients

$$
\varepsilon_{1}(\omega)^{-1} \partial_{x}^{2} u+\varepsilon_{2}(\omega)^{-1} \partial_{y}^{2} u+\omega^{2} \mu(\omega) u=0, \omega \in \mathbb{R}
$$

where $\varepsilon_{1}, \varepsilon_{2}, \mu$ have the meaning of dielectric permittivity and magnetic permeability. This class of models generalizes isotropic materials considered in [9], where authors use $\varepsilon_{1}(\omega)=\varepsilon_{2}(\omega)$. A notable example of such models includes a uniaxial cold plasma model in two dimensions [7]. In this work we will limit ourselves to socalled passive systems [21], i.e. systems for which $\varepsilon_{1,2}(\omega), \mu(\omega)$ satisfy $\operatorname{Im}\left(\omega \varepsilon_{i}(\omega)\right)>0, i=1,2, \operatorname{Im}(\omega \mu(\omega)) \geq 0$ for $\operatorname{Im} \omega>0$, and are analytic in the upper complex half-plane.

The new (to our knowledge) results obtained in this work include:

(1) a simple recipe of the construction of stable PMLs for passive anisotropic systems (1), even in the case when the coefficients in (1) correspond to non-local in time operators, or when the model (1) is dissipative. Our results extend the ideas of Bécache et al. [9] to a more general class of models;

(2) an easy to check constructive stability condition of the PML for a sub-class of problems (1) where $\varepsilon_{i}, i=1,2$ and $\mu$ satisfy additional requirements. More precisely, we show that the necessary condition of the stability of the PML formulated in [9] in terms of the directions of phase and group velocity can be rewritten in an easy-to-check form. Moreover, it is also sufficient for the stability of the PMLs. This extends the existing result [9] for isotropic materials.

An important difference between our work and [9] (as well as many other works on PMLs) is that we perform the analysis by using the Laplace transform in time, rather than working with plane waves which come from the Fourier analysis. This allows to avoid the discussion of the primary cause of the instabilities of the PMLs, namely the presence of so-called backward propagating waves; an interested reader may consult [9] and [10]. To our knowledge, in very few works the PML had been studied in the Laplace domain setting, see e.g. articles for the well-posedness of non-dispersive systems by Halpern et al. [22] or for convergence of the radial PML by Chen [23]. Such an analysis allows to obtain quite easily sufficient conditions of stability of the PML, while it is easier to derive necessary stability conditions using plane wave techniques.

This article is organized as follows. In Section 1 we discuss in more detail a class of problems of interest, providing some important examples. Next, in Section 2 we connect the passivity requirement to the stability of the problem (1) in the time domain. In Section 3 we discuss the construction of stable PMLs for general passive materials. In the second part of the article, we concentrate on a subclass of materials, considered in [9], and characterized in Section 4. Based on the properties of these materials and the necessary stability condition of [9], we provide a criterion of the stability of PMLs for (1) in Section 5.

We illustrate our results with the numerical experiments in Sections 3.1, 3.3 and 5.4.

\section{Problem Setting}

We consider a problem of the wave propagation in dispersive, anisotropic media, which is described by the Maxwell equations. In particular, in the time domain (using the scaling $\varepsilon_{0}=\mu_{0}=c=1$ ) in $\mathbb{R}^{2}$ it reads

$$
\begin{aligned}
& \partial_{t} D_{x}=\partial_{y} H_{z} \\
& \partial_{t} D_{y}=-\partial_{x} H_{z} \\
& \partial_{t} B_{z}=-\partial_{x} E_{y}+\partial_{y} E_{x}
\end{aligned}
$$

The relation between the fields $\mathbf{D}$ and $\mathbf{E}$, and $H_{z}$ and $B_{z}$ is not necessarily explicitly known in the time domain, but often is given in the Laplace domain. For simplicity, we will consider the above problem with nonvanishing source terms, but zero initial conditions. We will denote by $s$ the Laplace variable, by $\hat{u}=\mathcal{L} u$ the Laplace transform of $u$ in time, and by $\mathbb{C}_{+}$the right complex half-plane: $\mathbb{C}_{+}=\{s \in \mathbb{C}: \operatorname{Re} s>0\}$. Additionally, given 
a function $f(s): \mathbb{C}_{+} \cup i \mathbb{R} \rightarrow \mathbb{C}$, we will denote

$$
\tilde{f}(\omega)=f(-i \omega), \operatorname{Im} \omega \geq 0
$$

Then the following identities hold true: $\hat{\mathbf{D}}=\underline{\underline{\varepsilon}}(s) \hat{\mathbf{E}}, \hat{H}_{z}=\mu^{-1}(s) \hat{B}_{z}$. In the time domain we rewrite them as the following convolutions (where $\varepsilon\left(\partial_{t}\right)$ denotes a time-domain distribution with the symbol $\varepsilon(s)$ ):

$$
\mathbf{D}=\underline{\underline{\varepsilon}}\left(\partial_{t}\right) \mathbf{E}, \quad B_{z}=\mu\left(\partial_{t}\right) H_{z} .
$$

In particular, we concentrate on the case when the tensor of dielectric permittivity is diagonal. In this case the Maxwell's equations are of the following form:

$$
\operatorname{curl} \mu^{-1}(s) \operatorname{curl} \hat{\mathbf{E}}+s^{2}\left(\begin{array}{cc}
\varepsilon_{1}(s) & 0 \\
0 & \varepsilon_{2}(s)
\end{array}\right) \hat{\mathbf{E}}=0
$$

or, alternatively, cf. (1),

$$
s^{2} \mu(s) \hat{H}_{z}-\varepsilon_{2}(s)^{-1} \partial_{x}^{2} \hat{H}_{z}-\varepsilon_{1}(s)^{-1} \partial_{y}^{2} \hat{H}_{z}=0 .
$$

Let us provide a few examples of such models, and comment on the state of the art of the PMLs for those:

(1) isotropic Drude materials:

$$
\varepsilon_{1}(s)=\varepsilon_{2}(s)=1+\frac{\omega_{e}^{2}}{s^{2}}, \quad \mu(s)=1+\frac{\omega_{m}^{2}}{s^{2}}, \quad \omega_{e}, \omega_{m} \geq 0
$$

The corresponding system in the time domain reads, cf. [9],

$$
\begin{aligned}
& \partial_{t} \mathbf{E}-\operatorname{curl} H_{z}+\omega_{e}^{2} \mathbf{J}=0, \\
& \partial_{t} H_{z}+\operatorname{curl} \mathbf{E}+\omega_{m}^{2} K=0, \\
& \partial_{t} \mathbf{J}=\mathbf{E}, \\
& \partial_{t} K=H_{z} .
\end{aligned}
$$

The construction of the stable PMLs for this class of models in the case $\omega_{e}=\omega_{m}$ was suggested in [8]. In [9] the authors have extended these ideas to more general cases.

(2) uniaxial cold plasma model:

$$
\varepsilon_{1}(s)=1+\frac{\omega_{p}^{2}}{s^{2}}, \omega_{p}>0, \varepsilon_{2}(s)=\mu(s)=1 .
$$

In the time domain the corresponding system reads

$$
\begin{aligned}
& \partial_{t} E_{x}-\partial_{y} H_{z}+j=0, \\
& \partial_{t} E_{y}+\partial_{x} H_{z}=0, \\
& \partial_{t} H_{z}+\partial_{x} E_{y}-\partial_{y} E_{x}=0, \\
& \partial_{t} j=\omega_{p}^{2} E_{x} .
\end{aligned}
$$

This model was considered in $[6,7]$. The stable PMLs were constructed using an idea similar to [9]. 
(3) generalized Lorentz materials, which generalize the previous two cases:

$$
\begin{array}{llll}
\varepsilon_{1}(s)=1+\sum_{\ell=0}^{n_{x}} \frac{\varepsilon_{x \ell}}{s^{2}+\omega_{x \ell}^{2}}, & \varepsilon_{x \ell}>0, & \omega_{x \ell} \in \mathbb{R}, & \ell=0, \ldots, n_{x}, \\
\varepsilon_{2}(s)=1+\sum_{\ell=0}^{n_{y}} \frac{\varepsilon_{y \ell}}{s^{2}+\omega_{y \ell}^{2}}, & \varepsilon_{y \ell}>0, & \omega_{y \ell} \in \mathbb{R}, & \ell=0, \ldots, n_{y}, \\
\mu(s)=1+\sum_{\ell=0}^{n_{\mu}} \frac{\mu_{\ell}}{s^{2}+\omega_{\mu \ell}^{2}}, & \mu_{\ell}>0, & \omega_{\mu \ell} \in \mathbb{R}, & \ell=0, \ldots, n_{\mu} .
\end{array}
$$

The corresponding system of equations can be constructed similarly to (7). For the case $\varepsilon_{1}(s)=\varepsilon_{2}(s)$, the stable PMLs for this system have been constructed in [9], by extending and justifying mathematically the idea of [8]. A major part of the present work will be devoted to extending the results of this work to an anisotropic case $\varepsilon_{1} \neq \varepsilon_{2}$. Moreover, the results of this article show how to construct the PML in a more general case, for example, in the presence of losses, when $\varepsilon_{2}(s)$ is defined as

$$
\varepsilon_{2}(s)=1+\sum_{\ell=0}^{n_{y}} \frac{\varepsilon_{y \ell}}{s^{2}+2 \nu_{\ell} s+\omega_{y \ell}^{2}}, \quad \nu_{\ell}>0, \quad \ell=0, \ldots, n_{y} .
$$

To our knowledge, stable PMLs for this case did not exist even for isotropic dispersive materials.

For other examples of passive materials we refer an interested reader to [24].

Remark 1.1. Under extra assumptions (causality, analyticity and certain bounds on the coefficients), (5) with zero initial conditions can be rewritten in the time domain as

$$
\int_{0}^{t} \mathcal{M}(t-\tau) \partial_{\tau \tau} H_{z}(\tau) d \tau-\int_{0}^{t} \mathcal{E}_{2}(t-\tau) \partial_{x x} H_{z}(\tau) d \tau-\int_{0}^{t} \mathcal{E}_{1}(t-\tau) \partial_{y y} H_{z}(\tau) d \tau=0, t \geq 0
$$

where $\mathcal{M}=\mathcal{L}^{-1} \mu, \mathcal{E}_{1,2}=\mathcal{L}^{-1} \varepsilon_{1,2}^{-1}$

\section{Properties of the Dielectric Permittivity and the Magnetic Permeability}

In the physics literature, when considering the Maxwell equations (2) in so-called passive media, one assumes that $\varepsilon_{1,2}$ and $\mu$ satisfy the following assumptions (here we list only those which are of interest for us) [21,25]:

$(\mathrm{P} 1) \underline{\underline{\varepsilon}}(s), \mu(s)$ are analytic in $s \in \mathbb{C}_{+}$.

(P2) passivity, which we will use in the form

$$
\operatorname{Re}\left(s \varepsilon_{j}(s)\right)>0, j=1,2, \operatorname{Re}(s \mu(s))>0, s \in \mathbb{C}_{+} .
$$

Let us remark that in [21] this requirement is formulated (up to a complex rotation) as $\operatorname{Re}\left(s\left(\varepsilon_{j}(s)-1\right)\right) \geq$ 0 in $\mathbb{C}_{+}$; we use a slightly relaxed version. ${ }^{1}$

\footnotetext{
${ }^{1}$ In another reference [25], one considers materials with $\operatorname{Re}\left(s \varepsilon_{j}(s)\right) \geq 0, s \in \mathbb{C}_{+}$. Due to the maximum principle, applied to an analytic function $\mathrm{e}^{-s \varepsilon_{j}(s)}$, if in some point $s$, the real part $\operatorname{Re}\left(s \epsilon_{j}(s)\right)=0$, then $s \varepsilon_{j}(s)=$ const; one deduces $s \varepsilon_{j}(s)=i \alpha, \alpha \in \mathbb{R}$. This case is not of interest, since in the time domain the operator with the symbol $s \epsilon_{j}(s)$ would map real fields into complex fields, which is non-physical.
} 
Although we concentrate our presentation on the wave propagation problems (2), the model (5), which we will study in the present work, describes other phenomenae, for example, heat transfer (with a formal choice $\left.\varepsilon_{1}(s)=\varepsilon_{2}(s)=1, \mu(s)=s^{-1}\right)$.

This motivates us to redefine separately the property of passivity.

Definition 2.1. We will call a function $c: \mathbb{C}_{+} \rightarrow \mathbb{C}$ passive if it is analytic in $\mathbb{C}_{+}$and satisfies $\operatorname{Re}(s c(s))>0$ in $\mathbb{C}_{+}$.

We proceed as follows. First we recall some known properties of functions that satisfy (11), and next show that (P1) and (P2) are sufficient for the well-posedness of the problem (5).

\subsection{Properties of Analytic Functions Satisfying the Passivity Requirement}

Here we will provide a few known results on functions passive in the sense of Definition 2.1. Given such a function $c(s)$, notice that the function $f_{c}(z):=z c(-i z)$ with $z$ satisfying $\operatorname{Im} z>0$ is a Herglotz function (i.e. it is holomorphic in the upper half-plane $\operatorname{Im} z>0$, and $\operatorname{Im} f(z) \geq 0$ there). This can be seen by setting $z=i s$ :

$$
\operatorname{Im}(z c(-i z))=\operatorname{Re}(-i z c(-i z))=\operatorname{Re}(s c(s)) .
$$

The class of analytic in $\mathbb{C}_{+}$functions $g(s)$ which satisfy $\operatorname{Re} g(s)>0$ in $\mathbb{C}_{+}$, with some additional constraints (among them, in particular, $g(\bar{s})=\overline{g(s)}$ ) is known also as positive real functions, see the works by Brune [26] or Richards [27], where a concise summary of their properties can be found.

The following lemma provides bounds on the functions satisfying the passivity requirement. The upper bound in this lemma can be obtained using [28, Theorem 8', p.18], and the lower bound can be viewed as a minor improvement of [27, Theorem 3] (we consider a more general class of functions, however, the main idea of the proof is basically the same).

Lemma 2.2. Let $c(s)$ be passive in the sense of Definition 2.1. Then

(1) there exists $C>0$, s.t. for $s \in \mathbb{C}_{+}$, the function $c(s)$ satisfies

$$
|c(s)| \leq C|s|(\operatorname{Re} s)^{-1} \max \left(1,(\operatorname{Re} s)^{-2}\right) .
$$

(2) there exists $c>0$, s.t. for all $s \in \mathbb{C}_{+}$, it holds that

$$
\operatorname{Re}(s c(s))>c|s|^{-2} \min \left(1,(\operatorname{Re} s)^{2}\right) \operatorname{Re} s .
$$

The proof of the above lemma can be found in Appendix A.

Remark 2.3. Notice that passive functions $c(s)$ are not necessarily defined on the imaginary axis, a simple example being $c(s)=\frac{\log (i s)}{i s}$, which has a branch cut on the positive imaginary semiaxis $\{s: \operatorname{Im} s>0$, $\operatorname{Re} s=$ $0\}$.

From the above we immediately obtain a similar result for a function $b(s)$ which satisfies $\operatorname{Re}(\bar{s} b(s))>0$ in $\mathbb{C}_{+}$. Notice that given an analytic in $\mathbb{C}_{+}$function $b(s)$

$$
\operatorname{Re}(\bar{s} b(s))>0, s \in \mathbb{C}_{+} \Longleftrightarrow \operatorname{Re}\left(\frac{b(s)}{s}\right)>0, s \in \mathbb{C}_{+} \Longleftrightarrow \operatorname{Re}\left(\frac{s}{b(s)}\right)>0, s \in \mathbb{C}_{+}
$$

The function $\frac{1}{b(s)}$ is well-defined in $\mathbb{C}_{+}$, since $b(s)$ does not vanish there. With the help of the above, we obtain the following simple result on the behaviour of such a function $b(s)$.

Corollary 2.4. Let $b(s)$ be analytic in $\mathbb{C}_{+}$and $\operatorname{Re}(\bar{s} b(s))>0$ there. Then 
(1) there exists $C>0$, s.t. for $s \in \mathbb{C}_{+}$, it holds

$$
|b(s)| \leq C|s|^{3}(\operatorname{Re} s)^{-1} \max \left(1,(\operatorname{Re} s)^{-2}\right)
$$

(2) there exists $c>0$, s.t. for $s \in \mathbb{C}_{+}$

$$
\operatorname{Re}(\bar{s} b(s)) \geq c \min \left(1,(\operatorname{Re} s)^{2}\right) \operatorname{Re} s
$$

Proof. The upper and lower bounds can be obtained simply by noticing that $g_{b}(s):=b(s) s^{-2}$ satisfies Re $\left(s g_{b}(s)\right)=$ $\operatorname{Re}\left(\bar{s} \frac{b(s)}{|s|^{2}}\right)>0$ in $\mathbb{C}_{+}$. Then both bounds follow immediately from $(12,13)$ applied to $g_{b}(s)$. Namely,

$$
|b(s)|=|s|^{2}\left|g_{b}(s)\right| \leq C|s|^{3}(\operatorname{Re} s)^{-1} \max \left(1,(\operatorname{Re} s)^{-2}\right), C>0, \quad \operatorname{Re}(\bar{s} b(s)) \geq c \min \left(1,(\operatorname{Re} s)^{2}\right) \operatorname{Re} s, c>0
$$

The following lemma summarizes some of the results on the behaviour of functions $h(s)$ such that Re $h(s)>0$ for all $s \in \mathbb{C}_{+}$on the imaginary axis, and is essentially a reformulation of results of [26] (the first statement of the lemma below), [28, p.308, Theorem 1](the second statement of the lemma for $h(s)$ purely imaginary on $i \mathbb{R}),[29$, Proposition 13.8.1, p.920] (the second statement of the lemma, in a slightly modified form).

Lemma 2.5. Let $h(s)$ be analytic in $\mathbb{C}_{+}$and $\operatorname{Re} h(s)>0$ there. Then

(1) poles and zeros of $h(s)$ that lie on the imaginary axis are simple. Residues of the function $h(s)$ of its poles on the imaginary axis are real, positive, and so are the derivatives of $h(s)$ in its zeros on the imaginary axis.

(2) if $h(s)$ is additionally meromorphic in $\mathbb{C}$, and $h(-\bar{s})=-\overline{h(s)}$, all the poles and zeros of $h(s)$ lie on the imaginary axis and they alternate.

Proof. (1) let us first consider the case of zeros. Assume that $h(s)$ has a zero $s_{0}=i \omega_{0}$ of multiplicity $m$ on the imaginary axis. Then in a small vicinity of $i \omega_{0}$, with $s=i \omega_{0}+\varepsilon \mathrm{e}^{i \phi}$ and $\varepsilon \rightarrow 0$ :

$$
h\left(i \omega_{0}+\varepsilon \mathrm{e}^{i \phi}\right)=\frac{h^{(m)}\left(i \omega_{0}\right)}{m !} \varepsilon^{m} \mathrm{e}^{i m \phi}+O\left(\varepsilon^{m+1}\right), \phi \in(-\pi, \pi] .
$$

Assume $h^{(m)}\left(i \omega_{0}\right)=r \mathrm{e}^{i \phi_{0}}, r>0$, for some $\phi_{0} \in(-\pi, \pi]$. For $\phi \in\left(-\frac{\pi}{2}, \frac{\pi}{2}\right)$, i.e. when $\operatorname{Re} s>0$, it holds

$$
\operatorname{Re} h(s)=\frac{r \varepsilon^{m}}{m !} \cos \left(m \phi+\phi_{0}\right)+O\left(\varepsilon^{m+1}\right)>0
$$

from which it follows that, necessarily, $\cos \left(m \phi+\phi_{0}\right)>0$ for all $\phi \in\left(-\frac{\pi}{2}, \frac{\pi}{2}\right)$. This would be impossible if $m$ were larger than 1 , or $m=1$ and $\phi_{0} \neq 0$. Hence, $m=1$ and $\phi_{0}=0$.

In particular, we immediately obtain $h^{\prime}\left(i \omega_{0}\right)=r>0$ in a zero $s_{0}=i \omega_{0}$.

The condition on the poles, and thus, the residues of $h$, can be obtained by looking at the principal part of the Laurent series.

(2) if $h(s)=-\overline{h(-\bar{s})}$, and $h(s)$ has a pole with $\operatorname{Re} s<0,-\bar{s} \in \mathbb{C}_{+}$is correspondingly its pole as well. This is a contradiction to the analyticity of $h(s)$. On the other hand, if $h(s)$ a zero with Re $s<0$, the existence of zero $-\bar{s} \in \mathbb{C}_{+}$contradicts the condition $\operatorname{Re} h(s)>0$ in $\mathbb{C}_{+}$combined with the maximum principle, see the footnote on p. 4.

Next, assume that $h(s)$ has $m>1$ zeros (for simplicity let $m=2$ ), which lie on the imaginary axis and between which there is no pole. Let us take a contour $\gamma$, which is symmetric with respect to the 
imaginary axis and encloses these two zeros and no other zeros/poles of $h(s)$, and on which $h(s)$ is analytic. Thanks to the argument principle,

$$
\int_{\gamma} \frac{h^{\prime}(s)}{h(s)} d s=4 \pi i
$$

i. e. the change of the argument of $h(s)$ along the contour $\gamma$ equals to $4 \pi$. Denoting by $\gamma^{-}$a part of the contour with $\operatorname{Re} s<0$ and by $\gamma^{+}$the part of the contour with $\operatorname{Re} s>0$, we notice that for $s \in \gamma^{+}$, $-\bar{s} \in \gamma^{-}$, due to the symmetry. Moreover, $h(s)=-\overline{h(-\bar{s})}$, and thus the change of the argument of $h(s)$ along $\gamma_{+}$equals $2 \pi$, and so does the change of the argument of $h(s)$ along $\gamma_{-}$.

Since $\gamma^{+} \in \mathbb{C}_{+}$, this is a contradiction to $\operatorname{Re} h(s)>0$ in $\mathbb{C}_{+}$.

A similar argument can be applied to show that among two consecutive poles of $h(s)$ on the imaginary axis there is always a zero.

Thus, a passive function $c(s)$ cannot vanish in zero (otherwise $s c(s)$ would have a zero of multiplicity two there).

\subsection{Passivity Implies Stability: Laplace Domain Analysis}

The goal of this section is to investigate a connection between the passivity and the stability of the Maxwell system (5). The main result of this section is Theorem 2.15 formulated in the end of the section.

For convenience, let us introduce a sesquilinear form $A(u, v): H^{1}\left(\mathbb{R}^{2}\right) \times H^{1}\left(\mathbb{R}^{2}\right) \rightarrow \mathbb{C}$ :

$$
A(u, v)=a(s)\left(\partial_{x} u, \partial_{x} v\right)+b(s)\left(\partial_{y} u, \partial_{y} v\right)+s^{2} c(s)(u, v), s \in \mathbb{C}_{+}, u, v \in H^{1}\left(\mathbb{R}^{2}\right) .
$$

Here

$$
(u, v)=\int_{\mathbb{R}^{2}} u(x) \bar{v}(x) d x, \quad \text { for } u, v \in L^{2}\left(\mathbb{R}^{2}\right) .
$$

Given $f \in H^{-1}\left(\mathbb{R}^{2}\right), u \in H^{1}\left(\mathbb{R}^{2}\right)$, we will denote by $\langle f, u\rangle$ the duality pairing induced by the above inner product (where $H^{-1}\left(\mathbb{R}^{2}\right)$ is a space of antilinear functionals on $H^{1}\left(\mathbb{R}^{2}\right)$ ).

Remark 2.6. The operator corresponding to (18) is defined as follows

$$
\begin{aligned}
& \mathcal{A}_{s}: \quad H^{1}\left(\mathbb{R}^{2}\right) \rightarrow H^{-1}\left(\mathbb{R}^{2}\right), \\
& \mathcal{A}_{s} u=-a(s) \partial_{x}^{2} u-b(s) \partial_{y}^{2} u+s^{2} c(s) u .
\end{aligned}
$$

Indeed, we recognize the problem (5) with

$$
a(s)=\varepsilon_{2}(s)^{-1}, b(s)=\varepsilon_{1}(s)^{-1}, c(s)=\mu(s) .
$$

Remark 2.7. The case $a(s)=b(s)$ corresponds to an isotropic medium, see also [9].

Remark 2.8. All the results of this section are valid for a bounded Lipschitz domain $\Omega$, with the corresponding modification of the spaces for which the sesquilinear form $A(u, v)$ is defined (e.g. $H_{0}^{1}(\Omega) \times H_{0}^{1}(\Omega)$ for the homogeneous Dirichlet problem).

An approach that we are going to adopt here is based on the Laplace transform, see e.g. [30]. It is possible to obtain some of the results on the stability of the systems presented in the following sections with the help of the plane-wave analysis [31, Chapter 2], which is discussed in Section 2.3.

The results used here are based on the theory developed in [32, Section 2.1], which was, under stronger assumptions, made more precise in the following theorem of Dominguez and Sayas [33]. We present here a 
less refined version of this theorem. Let us remark that by causal we mean distributions $\phi$ defined on $\mathbb{R}$, s.t. $\langle\phi, \lambda\rangle=0$, for all $\lambda: \operatorname{supp} \lambda \in(-\infty, 0)$.

Theorem 2.9 (Proposition 3.2.2 [34], Proposition 3.2.2 [35]). Let $X, Y$ be Banach spaces, and $f$ be an $\mathcal{L}(X, Y)$ valued causal distribution whose Laplace transform $F(s)$ exists for all $s \in \mathbb{C}_{+}$and satisfies

$$
\|F(s)\|_{\mathcal{L}(X, Y)} \leq C_{F}(\operatorname{Re} s)|s|^{\gamma}, s \in \mathbb{C}_{+}
$$

where $\gamma \geq 0$ and $C_{F}:(0, \infty) \rightarrow(0, \infty)$ is a non-increasing function, s.t. $C_{F}(\lambda)<C \sigma^{-m}, C>0, m \geq 0$, for $\sigma \in(0,1]$. Define $k=\lfloor\gamma+2\rfloor$ (where $\lfloor k\rfloor$ denotes the integer part of $k)$. Then for all causal $C^{k-1}(\mathbb{R})$ functions $g: \mathbb{R} \rightarrow X$ with integrable $k$-th distributional derivative, the distribution $f * g$ is a causal continuous function $\mathbb{R} \rightarrow Y$ which satisfies the following bound with $\alpha_{\gamma, m}>0$ independent of $t:$

$$
\|(f * g)(t)\|_{Y} \leq \alpha_{\gamma, m} \max \left(t^{m}, 1\right) \int_{0}^{t}\left\|\left(1+\partial_{\tau}\right)^{k} g(\tau)\right\|_{X} d \tau, \quad t \geq 0 .
$$

Proof. See [34, Proposition 3.2.2] for the proof. Notice that in the derivation of the above bounds we use $t^{\varepsilon}(1+$ $t)^{-\varepsilon} \leq \mathrm{const}$ for $\varepsilon \geq 0$ (see [34, Proposition 3.2.2] for the notation), and the bound $C_{F}(1 / t) \leq C \max \left(t^{m}, 1\right)$.

Remark 2.10. First of all, as remarked in [34, p. 45], the bounds in the above result are non-optimal. However, provided e.g. a compactly supported right hand side data, the bound in (22) grows not faster than a polynomial in the time domain, which implies the time-domain stability.

Now let us come back to the question of the well-posedness of the variational formulation with the sesquilinear form (18). We will need the following assumption on coefficients of the sesquilinear form (18).

Definition 2.11. We will call the sesquilinear form (18) passive if the functions $a(s)^{-1}, b(s)^{-1}, c(s)$ are passive in the sense of Definition 2.1.

Our goal in this section is to show that passive sesquilinear forms define stable systems in the time domain. This motivates the name 'passive', since for the Maxwell system (2) the passivity requirement is connected to stability [24]. First of all, a direct application of Theorem 2.9 provides the following result.

Proposition 2.12 (Properties of passive sesquilinear forms). Let the sesquilinear form A(u,v) given by (18) be passive in the sense of Definition 2.11. Then:

(1) for some $C_{0}, C_{1}>0$ independent of $s$, and for all $s \in \mathbb{C}_{+}$, it holds:

$$
\begin{gathered}
|A(u, v)| \leq C_{0}|s|^{3}(\operatorname{Re} s)^{-1} \max \left(1,(\operatorname{Re} s)^{-2}\right)\|u\|_{H^{1}}\|v\|_{H^{1}}, \quad u, v \in H^{1}\left(\mathbb{R}^{2}\right) \\
\operatorname{Re}\left(\mathrm{e}^{-i \operatorname{Arg} s} A(u, u)\right) \geq C_{1} \operatorname{Re} s|s|^{-1} \min \left(1,(\operatorname{Re} s)^{2}\right)\|u\|_{H^{1}}^{2}, \quad u \in H^{1}(\mathbb{R})
\end{gathered}
$$

(2) for all $G \in H^{-1}\left(\mathbb{R}^{2}\right)$, for all $s \in \mathbb{C}_{+}$, there exists a unique solution $U(s) \in H^{1}\left(\mathbb{R}^{2}\right)$ to the variational formulation

$$
A(U(s), v)=\langle G, v\rangle, \quad v \in H^{1}\left(\mathbb{R}^{2}\right)
$$

Moreover, there exists $C>0$, s.t. for $s \in \mathbb{C}_{+}$,

$$
\|U(s)\|_{H^{1}\left(\mathbb{R}^{2}\right)} \leq C|s|(\operatorname{Re} s)^{-1} \max \left(1,(\operatorname{Re} s)^{-2}\right)\|G\|_{H^{-1}\left(\mathbb{R}^{2}\right)}
$$

(3) let $G(s) \in H^{-1}\left(\mathbb{R}^{2}\right)$ be the Laplace transform of a causal $C^{2}$-function $g(t): \mathbb{R} \rightarrow H^{-1}\left(\mathbb{R}^{2}\right)$, whose $3 r d$ derivative in the sense of distributions $g^{(3)} \in L^{1}\left(\mathbb{R}, H^{-1}\left(\mathbb{R}^{2}\right)\right)$. Then the solution $U(s), s \in \mathbb{C}_{+}$, of 
(25) with $G=G(s)$ is the Laplace transform of a causal continuous function $u(t): \mathbb{R} \rightarrow H^{1}\left(\mathbb{R}^{2}\right)$, which can be bounded as follows, with $\alpha>0$ independent of $t$ :

$$
\|u(t)\|_{H^{1}} \leq \alpha \max \left(t^{3}, 1\right) \int_{0}^{t}\left\|\left(1+\partial_{\tau}\right)^{3} g(\tau)\right\|_{H^{-1}} d \tau, \quad t \geq 0 .
$$

Proof. First of all, let us show that $A(u, v)$ is bounded and coercive. Indeed, given $u, v \in H^{1}\left(\mathbb{R}^{2}\right)$, using (12) and (15), we obtain, for some $C_{0}>0$ independent of $s$ and for all $s \in \mathbb{C}_{+}$:

$$
|A(u, v)| \leq \max \left(|a(s)|,|b(s)|,|c(s)||s|^{2}\right)\|u\|_{H^{1}}\|v\|_{H^{1}} \leq C_{0}|s|^{3}(\operatorname{Re} s)^{-1} \max \left(1,(\operatorname{Re} s)^{-2}\right)\|u\|_{H^{1}}\|v\|_{H^{1}} .
$$

To show the coercivity, consider $u \in H^{1}\left(\mathbb{R}^{2}\right)$, and take $A(s u, u)=\bar{s} A(u, u)$, for $s \in \mathbb{C}_{+}$:

$$
\begin{aligned}
\operatorname{Re}(\bar{s} A(u, u)) & =\operatorname{Re}(\bar{s} a(s))\left\|\partial_{x} u\right\|_{L^{2}}^{2}+\operatorname{Re}(\bar{s} b(s))\left\|\partial_{y} u\right\|_{L^{2}}^{2}+|s|^{2} \operatorname{Re}(s c(s))\|u\|_{L^{2}}^{2} \\
& \geq \min \left(\operatorname{Re}(\bar{s} a(s)), \operatorname{Re}(\bar{s} b(s)),|s|^{2} \operatorname{Re}(s c(s))\right)\|u\|_{H^{1}}^{2} \geq C_{1} \min \left(1,(\operatorname{Re} s)^{2}\right) \operatorname{Re} s\|u\|_{H^{1}}^{2}, C_{1}>0,
\end{aligned}
$$

where the last inequality was obtained with the help of (13) and (16). The above coercivity estimate is obtained for $\bar{s} A(u, u)$, hence, after the division by $|s|$ both sides of the above inequality, we obtain the estimate (24).

The second statement is obtained by a direct application of the Lax-Milgram lemma.

To obtain the third statement, we use the invertibility of the operator (19). Let us take $s_{0} \in \mathbb{C}_{+}$, and define $T_{s}=\mathcal{A}_{s_{0}}^{-1} \mathcal{A}_{s}$ a bounded invertible operator from $H^{1}\left(\mathbb{R}^{2}\right)$ into $H^{1}\left(\mathbb{R}^{2}\right)$. Due to [36, p.592, Lemma 13], $T_{s}^{-1}$ is analytic in $\mathbb{C}_{+}$as an operator-valued function, which implies, thanks to [37, Propositions $\left.3.1,3.2\right]$, that $T_{s}^{-1}$, and thus $\mathcal{A}_{s}^{-1}$, is a Laplace transform of a causal distribution. Additionally, the bound $\left\|\mathcal{A}_{s}^{-1}\right\|_{H^{-1} \rightarrow H^{1}} \leq$ $C_{1}^{-1}|s|(\operatorname{Re} s)^{-1} \max \left(1,(\operatorname{Re} s)^{-2}\right)$ holds for $s \in \mathbb{C}_{+}$. Then the statement of the proposition follows by the application of Theorem 2.9 to $U(s)=\mathcal{A}_{s}^{-1} G(s)$ :

$$
\|u(t)\|_{H^{1}}=\left\|\left(\mathcal{L}^{-1} U(s)\right)(t)\right\|_{H^{1}} \leq \alpha \max \left(t^{3}, 1\right) \int_{0}^{t}\left\|\left(1+\partial_{\tau}\right)^{3} g(\tau)\right\|_{H^{-1}} d \tau, \quad t \geq 0, \quad \alpha>0 .
$$

Let us now apply the above results to the model (5) with source terms, more precisely,

$$
\begin{array}{ll}
\partial_{t} \mathbf{D}-\operatorname{curl} H_{z}=\mathbf{f}_{\mathbf{D}}, & \partial_{t} B_{z}+\operatorname{curl} \mathbf{E}=f_{z}, \\
\mathbf{D}=\underline{\underline{\varepsilon}}\left(\partial_{t}\right) \mathbf{E}, & B_{z}=\mu\left(\partial_{t}\right) H_{z} .
\end{array}
$$

Recall that the notation $\mu\left(\partial_{t}\right) H_{z}$ corresponds to the convolution

$$
\mu\left(\partial_{t}\right) H_{z}=\int_{0}^{t} M(t-\tau) H_{z}(\tau) d \tau
$$

where $M$ is the inverse Laplace transform of $\mu(s)$, see [38].

We define the regularity for the right-hand side, for any $k, m \in \mathbb{N}_{0}$ as follows:

$$
\begin{array}{ll}
\mathbf{f}_{D} \in C^{k}\left(\mathbb{R} ;\left(L^{2}\left(\mathbb{R}^{2}\right)\right)^{2}\right), & \mathbf{f}_{D}^{(k+1)} \in L^{1}\left(\mathbb{R} ;\left(L^{2}\left(\mathbb{R}^{2}\right)\right)^{2}\right), \\
f_{z} \in C^{m}\left(\mathbb{R} ; L^{2}\left(\mathbb{R}^{2}\right)\right), & f_{z}^{(m+1)} \in L^{1}\left(\mathbb{R} ; L^{2}\left(\mathbb{R}^{2}\right)\right) .
\end{array}
$$


Definition 2.13. We will call the problem (27) well-posed, if there exists $k_{*}, m_{*} \in \mathbb{N}_{0}$, s.t. for any causal righthand side data satisfying $\left(R_{k m}\right)$ for some $k \geq k_{*}$ and $m \geq m_{*}$, the problem (27) with zero initial conditions has a unique solution $\left(\mathbf{E}, H_{z}, \mathbf{D}, B_{z}\right) \in C^{0}\left(\mathbb{R} ; L^{2}\left(\mathbb{R}^{2}\right)\right)$, which satisfies

$$
\|\mathbf{E}(t)\|_{L^{2}}+\left\|H_{z}(t)\right\|_{L^{2}}+\|\mathbf{D}(t)\|_{L^{2}}+\left\|B_{z}(t)\right\|_{L^{2}} \leq C(t) \mathrm{e}^{a t} \int_{0}^{t}\left(\sum_{\ell=0}^{k}\left\|\mathbf{f}_{D}^{(\ell)}(\tau)\right\|_{L^{2}}+\sum_{\ell=0}^{m}\left\|f_{z}^{(\ell)}(\tau)\right\|_{L^{2}}\right) d \tau,
$$

where $a \geq 0, C(t)$ is polynomial in $t$ (i.e. $C(t) \leq c\left(1+t^{n}\right)$, for some $n, c \geq 0$ ). If, additionally, the above bound holds with $a=0$, we will call the problem (27) stable.

Remark 2.14. In our definition of the well-posedness, the couple $\left(k_{*}, m_{*}\right)$ corresponds to a minimal regularity required on the data in order to be able to obtain estimates of the solution via the Laplace domain technique. In general, this requirement is not optimal. The minimal regularity is related to the explicit form of $\underline{\underline{\varepsilon}}(s), \mu(s)$.

For the classical Maxwell equations, when $\varepsilon_{1}(s)=\varepsilon_{2}(s)=\mu(s)=1$, one has $k_{*}=m_{*}=2$.

While the regularity required by the Hille-Yosida theorem is lower, e.g. $\mathbf{f}_{D} \in C^{1}\left(\mathbb{R}_{+} ;\left(L^{2}\left(\mathbb{R}^{2}\right)\right)^{2}\right)$, the results of this work allow to obtain the explicit bounds on the solutions in terms of the right hand side data, unlike the result of Hille-Yosida.

Applying Proposition 2.12, we immediately obtain the following result.

Theorem 2.15 (Connection of the Passivity and the Stability). The problem (27) with passive diagonal dielectric permittivity and magnetic permeability is well-posed and stable.

Remark 2.16. In many practical cases $\varepsilon_{1}(s), \varepsilon_{2}(s)$ and $\mu(s)$ are rational fractions, cf. Lorentz materials (9). Then the convolutions in (27) can be computed by introducing auxiliary unknowns and coupling (27) with an ODE system for the corresponding unknowns, see e.g. the system (60a-60e). In a more general setting, this is explained in [24]. The result of Theorem 2.15 holds true in this case as well.

It would be natural to ask whether the passivity requirement is necessary for the stability of (27). This is true [24] for a class of isotropic dispersive models.

\subsection{Passivity Implies Stability: Plane-Wave Analysis}

In this section we will briefly discuss a connection between the plane-wave analysis and the Laplace domain analysis. A Fourier-based approach, see e.g. [31, Chapter 2], had been used to study the stability of the PMLs in isotropic dispersive media [9], or anisotropic non-dispersive media [10]. When applied to the system (5) without the PML, such analysis consists in looking for the plane-wave solutions $\hat{H}_{z} \mathrm{e}^{-i(\omega t-\mathbf{k} \cdot \mathbf{x})}, \mathbf{k}, \mathbf{x} \in \mathbb{R}^{2}, \omega \in \mathbb{C}$, of (5) rewritten in the time domain. This requires examining the corresponding dispersion relation, which, in general, depends on the time domain formulation of the problem. However, some of its solutions satisfy

$$
\tilde{\varepsilon}_{2}(\omega)^{-1} k_{x}^{2}+\tilde{\varepsilon}_{1}(\omega)^{-1} k_{y}^{2}-\omega^{2} \tilde{\mu}(\omega)=0 .
$$

Definition 2.17. Continuous branches of solutions $\omega(\mathbf{k})$ of the dispersion relation are called modes.

In the time domain the system (5) is stable if and only if all the modes $\omega(\mathbf{k})$ satisfy

$$
\operatorname{Im} \omega(\mathbf{k}) \leq 0, \quad \text { for all } \mathbf{k} \in \mathbb{R}^{2} .
$$

This holds true for passive sesquilinear forms.

Proposition 2.18 (Modal Analysis for Passive Systems). Let the sesquilinear form (18) associated to a, $b, c$ given in (20) be passive. Then all the solutions $\omega(\mathbf{k})$ of (29) satisfy (30), and therefore, correspond to stable modes of (5). 
Proof. Setting $s=-i \omega,(29)$ can be rewritten as

$$
s^{2} c(s)+b(s) k_{y}^{2}+a(s) k_{x}^{2}=0, \quad \mathbf{k} \in \mathbb{R}^{2} .
$$

After multiplication by $\bar{s}$ this yields:

$$
|s|^{2} s c(s)+\bar{s} b(s) k_{y}^{2}+\bar{s} a(s) k_{x}^{2}=0, \quad \mathbf{k} \in \mathbb{R}^{2} .
$$

The passivity assumption implies that the real part of the LHS of this equation is strictly positive for $s \in \mathbb{C}_{+}$. Thus, the above equation has no solutions in $\mathbb{C}_{+}$, and hence all solutions of (29) satisfy (30).

\section{Construction of Stable PMLs for General Passive Materials}

\subsection{A Brief Introduction into PMLs for Dispersive Media. Instability of Classical PMLs for Anisotropic Dispersive Models}

\subsubsection{Introduction into PMLs for Dispersive Media}

In this section we will briefly present the technique of PMLs, with a particular application to dispersive media. A detailed introduction into this technique can be found e.g. in [39] or [40]. There are at least two ways to apply the perfectly matched layers to the system (2). The first one is to use splitting of the time-domain system, as in seminal works by Bérenger $[1,2]$. The second way consists in the change of variables in the frequency domain, as reinterpreted by Chew et al. in [41], see also [42]. Such PMLs are called unsplit PMLs. We will adopt here the latter approach. More precisely, consider the following equation

$$
a(s) \partial_{x}^{2} u+b(s) \partial_{y}^{2} u-s^{2} c(s) u=0, \quad s \in \mathbb{C}_{+},(x, y) \in \mathbb{R}^{2} .
$$

Let the perfectly matched layer be located in the region $x \geq 0$. We assume that the above equation is valid for $x=\tilde{x} \in \mathbb{C}_{+}$, and introduce an analytic continuation of $u$ that we denote $U(\tilde{x}, y)$. Naturally, $U$ will satisfy the above equation, however, for $(\tilde{x}, y) \in \mathbb{C}_{+} \times \mathbb{R}$. Then choosing a parameterization of $\tilde{x}$ suggested in [9]

$$
\tilde{x}=\left\{\begin{array}{ll}
x+s^{-1} \psi(s) \int_{0}^{x} \sigma\left(x^{\prime}\right) d x^{\prime}, & x \geq 0, \\
x, & x<0,
\end{array} \quad \text { where } \sigma(x)= \begin{cases}\sigma(x) \geq 0, & x \geq 0, \\
0, & x<0,\end{cases}\right.
$$

and $\psi(s)$ is an analytic in $\mathbb{C}_{+}$function, we obtain the following system:

$$
a(s)\left(1+\frac{\sigma(x) \psi(s)}{s}\right)^{-1} \partial_{x}\left(\left(1+\frac{\sigma(x) \psi(s)}{s}\right)^{-1} \partial_{x} U\right)+b(s) \partial_{y}^{2} U-s^{2} c(s) U=0, \quad(x, y) \in \mathbb{R}^{2} .
$$

For $x<0$, the above system coincides with (31). The original and the PML systems are coupled via transmission conditions. Importantly, $u(x, y)=U(x, y)$ for $x<0$ by analytic continuation. The resulting system needs to be rewritten in the time domain, see Section 4.2.

Other PML directions can be treated similarly, and in the corners the change of multiple variables should be used. To truncate the perfectly matched layer at some $x=L>0$, since in practice it cannot be chosen infinitely long, zero Dirichlet or Neumann boundary conditions are used on the exterior boundary of the PML.

Classical PMLs (split or unsplit) correspond to the choice $\psi(s)=1$. The function $\psi(s)$ was introduced in $[8,9]$ in order to take into account the dispersive character of the equations. Indeed, as demonstrated in [9], for isotropic dispersive models classical PMLs can lead to instabilities in the time domain. We demonstrate that this is also the case for anisotropic dispersive models with the help of the following simple numerical experiment. 
Remark 3.1. Notice that in terms of stability (i.e. the absence of exponential blow-up), there is no difference between split and unsplit PMLs, as they both correspond to the same change of variables.

\subsubsection{Numerical Illustration: Instability of Classical PMLs for Anisotropic Dispersive Models}

Before providing numerical data, let us introduce some auxiliary notation, which we will use in all the numerical experiments in this section, as well as Sections 3.3, 5.4. First of all, we compute solutions to the problem (2) inside rectangular domains. Their physical (i.e. without the PML) dimensions in the direction $x$ and $y$ are $L_{x}$, and $L_{y}$. The width of the PML in $x$-direction is denoted by $L_{x}^{\sigma}$, and in $y$-direction by $L_{y}^{\sigma}$. The absorption parameters in directions $x$ and $y$ are $\sigma_{x}(x)$ and $\sigma_{y}(y)$, cf. (32). On all outer boundaries (physical boundary if no PML in one of the directions is used, or external boundary of the PML), we assume zero Dirichlet boundary conditions for the field $H_{z}$.

The time domain PML system has a structure similar to the system of equations (62-63), see Section 4.2.2 and Remark 4.11. We use zero initial conditions and a source $f(t, x, y)$ in the third equation of (62). The numerical resolution is done with the help of the Yee scheme [43] for dispersive models, where the dispersive terms are discretized by trapezoid rule, and non-dispersive part is computed with a leapfrog. In the scheme, we discretize the field $H_{z}$ on integer time and spatial steps. The time step is denoted by $\Delta t$, and the space step is $\Delta x=\Delta y$.

In all the experiments where we show a solution at different time steps, or compare the stable and unstable PMLs, we use the same color scale in all relevant figures.

Example 3.2 (Instability of classical PMLs for anisotropic Drude material). We model an anisotropic Drude material, which extends the isotropic model (6), where

$$
a(s)=\varepsilon_{2}(s)^{-1}=\left(1+\frac{\omega_{a}^{2}}{s^{2}}\right)^{-1}, \quad b(s)=\varepsilon_{1}(s)^{-1}=\left(1+\frac{\omega_{b}^{2}}{s^{2}}\right)^{-1}, \quad \mu(s)=1 .
$$

In particular, we choose $\omega_{a}=8, \omega_{b}=4$. The passivity of the parameters can be checked by a direct calculation.

We use the classical PMLs in the direction $x$, i.e. $\psi(s)=1$ in (32), and zero Dirichlet BCs in the direction $y$, in order to show that the instability occurs not necessarily in the corner, but in a certain direction. The rest of the parameters can be found in Table 1 . We show the field $H_{z}$ computed with the help of the classical

$$
\begin{array}{ccccccccr}
L_{x} & L_{y} & L_{x}^{\sigma} & L_{y}^{\sigma} & \sigma_{x}(x) & \sigma_{y}(y) & \Delta x & \Delta t & f(t, x, y) \\
\hline 8 & 24 & 2 & 0 & 20 x^{2} & 0 & 0.05 & 0.025 & 10(t-1.6) \mathrm{e}^{-15(t-1.6)^{2}} \mathrm{e}^{-300 x^{2}-300 y^{2}}
\end{array}
$$

TABle 1. Parameters for the experiment with dielectric permittivity and magnetic permeability (34). The notation can be found in the beginning of Section 3.1.2.

PMLs in Figure 1. One can see the instability developing in this case. In the rightmost plot we demonstrate an exponential blow-up of the $L^{2}$-norm of the solution $H_{z}$.

\subsection{Construction of Stable PMLs}

\subsubsection{The Well-Posedness of the PMLs}

Let us consider the PML system in the Laplace domain (33). We associate to this equation the sesquilinear form

$$
\begin{aligned}
A_{p}(u, v) & =a(s)\left(\left(1+\frac{\sigma(x) \psi(s)}{s}\right) \partial_{x} u, \partial_{x} v\right)+b(s)\left(\left(1+\frac{\sigma(x) \psi(s)}{s}\right) \partial_{y} u, \partial_{y} v\right) \\
& +s^{2} c(s)\left(\left(1+\frac{\sigma(x) \psi(s)}{s}\right) u, v\right), \quad u, v \in H_{0}^{1}(\Omega) .
\end{aligned}
$$




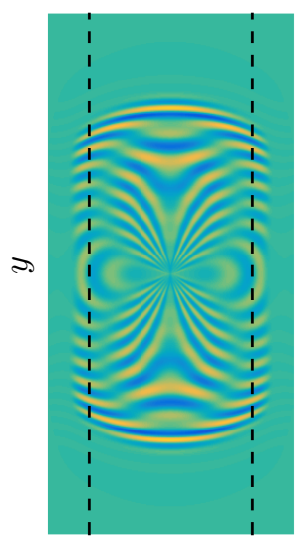

$x$

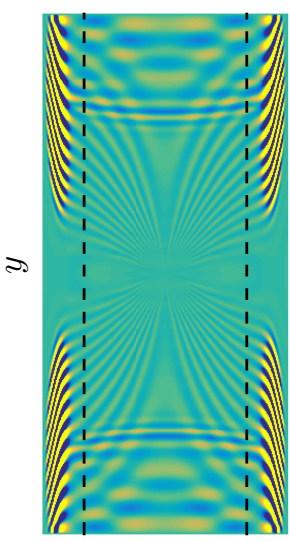

$x$

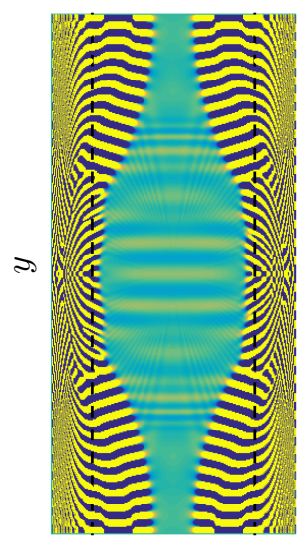

$x$

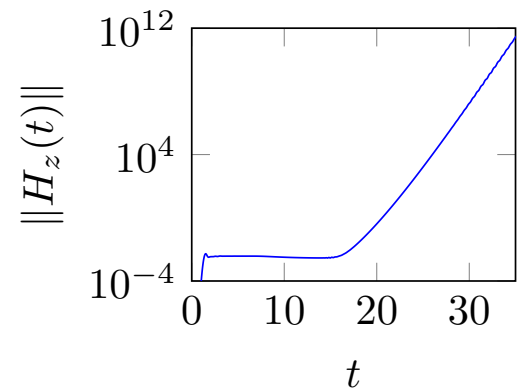

Figure 1. From left to right: a solution $H_{z}$ of Example 3.2 computed with the classical PML at $t=10,20,35$; the dependence of the $L^{2}$-norm of $H_{z}$ with respect to time. The boundary between the PML and the physical domain is marked in black.

To show the well-posedness of the corresponding time domain model, cf. (27), it suffices to demonstrate the coercivity of the above sesquilinear form for all $s \in \mathbb{C}_{s_{r}}=\left\{s \in \mathbb{C}: \operatorname{Re} s>s_{r}\right\}, s_{r} \in \mathbb{R}$.

One may notice that the sesquilinear form (35) is a particular case of the following sesquilinear form.

$$
A_{g}(u, v)=\left(\alpha(s, x, y) \partial_{x} u, \partial_{x} v\right)+\left(\beta(s, x, y) \partial_{y} u, \partial_{y} v\right)+s^{2}(\gamma(s, x, y) u, v), \quad u, v \in H_{0}^{1}(\Omega) .
$$

We will assume that for large Re $s$, the above sesquilinear form behaves as the sesquilinear form for the nondispersive wave equation.

Proposition 3.3. Let $\alpha(s, x, y), \beta(s, x, y), \gamma(s, x, y)$ satisfy the following:

$$
\alpha(s, x, y), \beta(s, x, y), \gamma(s, x, y) \rightarrow 1
$$

as $\operatorname{Re} s \rightarrow+\infty$, uniformly in $x, y \in \mathbb{R}^{2}$ and $\operatorname{Im} s$. Moreover, let $\alpha(s, x, y), \beta(s, x, y), \gamma(s, x, y)$ be analytic in $s: \operatorname{Re} s>M_{*}$ for some $M_{*}>0$, and a.e. in $\mathbb{R}^{2}$.

Then there exists $M>M_{*}>0$, s.t.:

(1) the sesquilinear form $A_{g}(u, v)$ is bounded and coercive for all $s: \operatorname{Re} s>M$; moreover, it holds

$$
\begin{array}{r}
\left|A_{g}(u, v)\right|<C|s|^{2}|u|_{H^{1}}|v|_{H^{1}}, \\
\operatorname{Re}\left(\bar{s} A_{g}(u, u)\right) \geq c_{0} \operatorname{Re} s|u|_{H^{1}}^{2}, \quad C, c_{0}>0 .
\end{array}
$$

(2) for all $G \in H^{-1}\left(\mathbb{R}^{2}\right)$, for all $s \in \mathbb{C}_{+}$, s.t. $\operatorname{Re} s>M$, there exists a unique solution $U(s) \in H^{1}\left(\mathbb{R}^{2}\right)$ to the variational formulation

$$
A_{g}(U(s), v)=\langle G, v\rangle, \quad v \in H^{1}\left(\mathbb{R}^{2}\right) .
$$

Moreover, for $s \in \mathbb{C}_{+}$,

$$
\|U(s)\|_{H^{1}\left(\mathbb{R}^{2}\right)} \leq C(\operatorname{Re} s)^{-1}|s|\|G(s)\|_{H^{-1}\left(\mathbb{R}^{2}\right)}, C>0 .
$$


(3) let $G(s) \in H^{-1}\left(\mathbb{R}^{2}\right)$ be the Laplace transform of a causal $C^{2}$-function $g(t): \mathbb{R} \rightarrow H^{-1}\left(\mathbb{R}^{2}\right)$, which satisfies $\left(\mathrm{e}^{-M t} g(t)\right)^{(3)} \in L^{1}\left(\mathbb{R}, H^{-1}\left(\mathbb{R}^{2}\right)\right)$. Then the solution $U(s), s \in \mathbb{C}_{+}$, of (37) with $G=G(s)$ is a Laplace transform of a causal continuous function $u(t): \mathbb{R} \rightarrow H^{1}\left(\mathbb{R}^{2}\right)$, which can be bounded as follows:

$$
\|u(t)\|_{H^{1}} \leq \alpha \max \left(t^{3}, 1\right) \mathrm{e}^{M t} \int_{0}^{t}\left\|\left(1+\partial_{\tau}\right)^{3}\left(\mathrm{e}^{-M \tau} g(\tau)\right)\right\|_{H^{-1}} d \tau, \quad t \geq 0, \quad \alpha>0 .
$$

Proof. Due to the properties of the coefficients of the sesquilinear form at the infinity, there exists $M>1$ sufficiently large so that for all $s$ with $\operatorname{Re} s>M$, it holds

$$
\begin{array}{r}
|\alpha(s, x, y)|_{\infty}<C_{\alpha},|\beta(s, x, y)|_{\infty}<C_{\beta},\left|s^{2} \gamma(s, x, y)\right|_{\infty}<C_{\gamma}|s|^{2}, \\
\operatorname{Re}(\bar{s} \alpha(s, x, y)) \geq c_{\alpha} \operatorname{Re} s, \operatorname{Re}(\bar{s} \beta(s, x, y)) \geq c_{\beta} \operatorname{Re} s, \operatorname{Re}\left(\bar{s} s^{2} \gamma(s, x, y)\right) \geq c_{\gamma}|s|^{2} \operatorname{Re} s \geq c_{\gamma} \operatorname{Re} s,
\end{array}
$$

with some constants $C_{\alpha}, C_{\beta}, C_{\gamma}, c_{\alpha}, c_{\beta}, c_{\gamma}>0$. From this the continuity and the coercivity bounds on the sesquilinear form follow.

The existence of the solution and the corresponding bound hold immediately from the Lax-Milgram theorem.

To come back to the time domain, let us consider the problem (37) and recast it into the known framework of problems defined for $s \in \mathbb{C}_{+}$. More precisely, for $\tilde{s} \in \mathbb{C}_{+}$, the bound (38) becomes:

$$
\begin{aligned}
\|U(\tilde{s}+M)\|_{H^{1}\left(\mathbb{R}^{2}\right)} & \leq C(M+\operatorname{Re} \tilde{s})^{-1}|\tilde{s}+M|\|G(\tilde{s}+M)\|_{H^{-1}\left(\mathbb{R}^{2}\right)} \\
& =C(M+\operatorname{Re} \tilde{s})^{-1} \sqrt{(\operatorname{Re} \tilde{s}+M)^{2}+(\operatorname{Im} \tilde{s})^{2}}\|G(\tilde{s}+M)\|_{H^{-1}\left(\mathbb{R}^{2}\right)} \\
& \leq C|\tilde{s}|\|G(\tilde{s}+M)\|_{H^{-1}\left(\mathbb{R}^{2}\right)}
\end{aligned}
$$

Thus, we are in the framework of Theorem 2.9, and proceed as in Proposition 2.12. In particular, we notice that given a function $g(t)$ with the Laplace transform $G(\tilde{s})$, the inverse Laplace transform of $G(\tilde{s}+M)$ is $\mathrm{e}^{-M t} g(t)$. From this the bound of the theorem follows.

The assumptions on the behaviour of $\alpha, \beta, \gamma$ at infinity are introduced for technical reasons. They can be waived, however, this requires separate consideration of the corresponding equations.

Corollary 3.4. Let $a(s), b(s), c(s), \psi(s)$ satisfy the following:

$$
a(s), b(s), c(s), \psi(s) \rightarrow 1
$$

as $\operatorname{Re} s \rightarrow+\infty$ uniformly in $\operatorname{Im} s$. Let $\sigma(x) \in L^{\infty}\left(\mathbb{R}^{2}\right)$. Let also $a, b, c, \psi$ be analytic in $s \in \mathbb{C}_{+}$. Then the form $A_{p}(u, v)$ can be recast in the form $A_{g}(u, v)$ with $\alpha=a\left(1+\frac{\sigma \psi}{s}\right)^{-1}, \beta=b\left(1+\frac{\sigma \psi}{s}\right)$ and $\gamma=c\left(1+\frac{\sigma \psi}{s}\right)$, and the latter satisfies assumptions of Proposition 3.3.

Corollary 3.5. Let $\varepsilon_{x}(s), \varepsilon_{y}(s), \mu(s), \psi(s) \rightarrow 1$ as $\operatorname{Re} s \rightarrow+\infty$ uniformly in $\operatorname{Im} s$. Let $\psi(s) \in L^{\infty}\left(\mathbb{R}^{2}\right)$. Then the system (27) with $\varepsilon_{y}(s)=a(s)^{-1}\left(1+\frac{\sigma \psi}{s}\right), \varepsilon_{x}(s)=b(s)^{-1}\left(1+\frac{\sigma \psi}{s}\right)^{-1}$ and $\mu(s)=c(s)\left(1+\frac{\sigma \psi}{s}\right)$ is well-posed.

The requirement about the behaviour at the infinity of the parameters of the model is not too restrictive (though it might seem not entirely standard, cf. [24]), and is valid e.g. for the Lorentz/Drude materials, as well as their generalizations.

The result of the well-posedness of the PML model is indeed important. However, for the numerical aspect of the PML such analysis does not seem to be very sufficient. Indeed, according to this result, the Bérenger's PML applied to the problem (34) leads to a well-posed system of equations; yet, the system is unstable. Therefore, it is crucial to perform a stability analysis. 


\subsubsection{Stability Analysis}

The question of the stability is somewhat more subtle than the well-posedness. There exist very few works, which provide a full stability analysis of the PMLs for nonconstant absorption parameters even for the case of the classical perfectly matched layers applied to nondispersive anisotropic models. For example, in [44] the authors prove the stability and convergence result of the 2D classical PML for isotropic nondispersive wave equation and extend it to stable PMLs for the advective wave equation. In [23] some convergence estimates are provided for the radial PMLs for 2D acoustic wave equation.

Due to complexity of rigorous analysis, the construction of stable PMLs is often done in two stages:

- assuming that $\sigma(x)=\sigma=$ const $>0$ in $\mathbb{R}^{2}$ in (35) and performing the analysis for the resulting problem. This can be viewed as a very particular case of an arbitrary $\sigma(x) \geq 0$. Such a simplification was used, for example, in works $[9-11,45,46]$. It facilitates finding the right PML change of variables (in our case $\psi(s)$ ), which is potentially stable for variable $\sigma(x)$. And, as many numerical experiments show (including those in the present work), this analysis often results in stable PMLs for $\sigma(x) \neq$ const.

- proving that the PML constructed with the help of the simplified analysis is stable for a more general class of absorption parameters. This part is trickier, as we discussed before, and is an open question for most existing stabilized PMLs.

In this work we will concentrate on the first part of the analysis. For the newly constructed PML the full rigorous proof of the stability is the subject of future research, see, in particular, [47].

\subsubsection{Stability Analysis}

Let us consider a simplified case, when the absorption function is a non-negative constant and is defined in $\mathbb{R}$. This amounts to taking $\sigma(x)=\sigma=$ const in (33) and considering the corresponding sesquilinear form for $(x, y) \in \mathbb{R}^{2}$. Alternatively, this can be viewed as the following change of variables:

$$
x \rightarrow x\left(1+s^{-1} \psi(s) \sigma\right), \quad \sigma \geq 0, \quad x \in \mathbb{R} .
$$

As shown before, the choice $\psi(s)=1$ may lead to time-domain instabilities. Our goal is to provide stable choices of $\psi(s)$ for an arbitrary passive material. The main results of this section are Theorems 3.11 and 3.14.

Upon the application of (39), the sesquilinear form (18) is transformed to

$$
A_{\sigma}(u, v)=a(s)\left(1+\frac{\sigma \psi(s)}{s}\right)^{-2}\left(\partial_{x} u, \partial_{x} v\right)+b(s)\left(\partial_{y} u, \partial_{y} v\right)+s^{2} c(s)(u, v), s \in \mathbb{C}_{+}, \quad u, v \in H^{1}\left(\mathbb{R}^{2}\right) .
$$

We suggest that taking in the above $\psi(s)=a(s)$ produces a passive sesquilinear form. This result is generalized in Lemma 3.6 and is explained in detail in Theorem 3.11. Although Lemma 3.6 does not provide an explicit way to construct the function $\psi(s)$, it will play an important role in Section 5.

Lemma 3.6 (Sufficient stability condition for the PML (39)). Let the sesquilinear form $A(u, v)$ given by (18) be passive in the sense of Definition 2.11. Let an analytic $\psi(s): \mathbb{C}_{+} \rightarrow \mathbb{C}$ satisfy for all $s \in \mathbb{C}_{+}$

(S1) $\operatorname{Re}(\bar{s} \psi(s))>0$,

(S2) $\operatorname{Re}\left(\bar{s} \psi(s) a(s)^{-1} b(s)\right)>0$,

(S3) $\operatorname{Re}\left(s \psi(s) a(s)^{-1} c(s)\right)>0$.

Then for all $\sigma \geq 0$, the sesquilinear form

$$
\tilde{A}_{\sigma}(u, v)=\psi(s) a(s)^{-1} A_{\sigma}(u, v), \quad u, v \in H^{1}\left(\mathbb{R}^{2}\right),
$$

where $A_{\sigma}(u, v)$ is defined in (40), is passive. 
Proof. Notice that since $a(s)$ satisfies $\operatorname{Re}(\bar{s} a(s))>0, s \in \mathbb{C}_{+}$, it never vanishes in $\mathbb{C}_{+}$. Let us verify the conditions on the coefficients of the sesquilinear form (41) required by passivity. The first coefficient

$$
\operatorname{Re}\left(s \psi(s)^{-1}\left(1+\frac{\sigma \psi(s)}{s}\right)^{2}\right)=\operatorname{Re}\left(s \psi(s)^{-1}\right)+2 \sigma+\operatorname{Re}\left(s^{-1} \psi(s)\right)>0,
$$

for all $s \in \mathbb{C}_{+}$, thanks to (14) and (S1). It is obviously analytic in $\mathbb{C}_{+}$. The passivity condition on the rest of the coefficients is included explicitly into the conditions of the lemma.

Combining the above with Theorem 2.9 and Proposition 2.12, we obtain the following simple result of the time-domain stability of the PML system.

Corollary 3.7 (Stability bounds of the PML of Lemma 3.6). Let $A_{\sigma}(u, v)$ be defined by (40), and let $\psi(s)$ satisfy the conditions of Lemma 3.6 Let, additionally,

$$
\left|\frac{\psi(s)}{a(s)}\right| \leq|s|^{\gamma} C_{f}(\operatorname{Re} s), \quad s \in \mathbb{C}_{+},
$$

with $\gamma \geq-1$ and $C_{f}(\lambda):(0, \infty) \rightarrow(0, \infty)$ being non-increasing and satisfying $C_{f}(\lambda)<C_{0} \lambda^{-m}, C_{0}>0, m \geq 0$. Then the following holds true for the sesquilinear form (40):

(1) for all $G \in H^{-1}\left(\mathbb{R}^{2}\right)$, for all $s \in \mathbb{C}_{+}$, there exists a unique solution $U(s) \in H^{1}\left(\mathbb{R}^{2}\right)$ to the variational formulation

$$
A_{\sigma}(U(s), v)=\langle G, v\rangle, \quad v \in H^{1}\left(\mathbb{R}^{2}\right),
$$

which satisfies, for all $s \in \mathbb{C}_{+}$,

$$
\|U(s)\|_{H^{1}\left(\mathbb{R}^{2}\right)} \leq C|s|^{\gamma+1}(\operatorname{Re} s)^{-1} \max \left(1,(\operatorname{Re} s)^{-2}\right) C_{f}(\operatorname{Re} s)\|G\|_{H^{-1}\left(\mathbb{R}^{2}\right)},
$$

where $C>0$ is a constant independent of $s$;

(2) let $k=\lfloor\gamma+3\rfloor$, and $\ell=3+m$. Let $G(s) \in H^{-1}\left(\mathbb{R}^{2}\right)$ be the Laplace transform of a causal function $g(t): \mathbb{R} \rightarrow H^{-1}\left(\mathbb{R}^{2}\right)$, which belongs to $C^{k-1}\left(\mathbb{R} ; H^{-1}\left(\mathbb{R}^{2}\right)\right)$ and has an integrable $k$-th distributional derivative. Then the solution $U(s), s \in \mathbb{C}_{+}$, of (43) with $G=G(s)$ is a Laplace transform of a causal continuous function $u(t): \mathbb{R} \rightarrow H^{1}\left(\mathbb{R}^{2}\right)$, which can be bounded as follows, with $\alpha>0$ independent of $t$ :

$$
\left.\|u(t)\|_{H^{1}} \leq \alpha \max \left(t^{\ell}, 1\right) \int_{0}^{t} \|\left(1+\partial_{\tau}\right)^{k} g\right)(\tau) \|_{H^{-1}} d \tau, \quad t \geq 0 .
$$

Proof. The first statement follows directly from Proposition 2.12, applied to the following variational formulation, which is equivalent to (43):

$$
\tilde{A}_{\sigma}(U(s), v)=\psi(s) a(s)^{-1}\langle G, v\rangle, \quad v \in H^{1}\left(\mathbb{R}^{2}\right), s \in \mathbb{C}_{+} .
$$

Notice that $\tilde{A}_{\sigma}(u, v)$ is passive, according to Lemma 3.6. The second statement is obtained as in the proof of Proposition 2.12 ; it is important to notice that $\psi(s) a(s)^{-1}$ is analytic in $\mathbb{C}_{+}$. The bound in the time domain follows by the application of Theorem 2.9.

Remark 3.8. Indeed, if (42) holds with $\gamma<-1$, we can always bound $|s|^{\gamma} C_{f}(\operatorname{Re} s)=|s|^{-1}|s|^{1+\gamma} C_{f}(\operatorname{Re} s) \leq$ $|s|^{-1}(\operatorname{Re} s)^{\gamma+1} C_{f}(\operatorname{Re} s)$, which satisfies the conditions of Corollary 3.7. 
The time-domain bounds in Corollary 3.7 are non-optimal, since they are based on the extensive use of Proposition 2.12 and non-optimal bounds of Theorem 2.9. Therefore this result cannot be used to compare the bounds on the solution of the PML and non-PML system. Using a priori bounds on $\left|a(s) \psi(s)^{-1}\right|$ which can be computed with the help of Corollary 2.4, we obtain the following simple result.

Corollary 3.9 (A priori bounds for Corollary 3.7). Let $a(s)^{-1}, \psi(s)^{-1}$ be passive. Then the estimate (42) holds true with $\gamma \leq 4, m \leq 6$. If additionally $a(s)^{-1}, \psi(s)^{-1}$ are generalized Lorentz, i.e. have an expansion as in (9), the estimate (42) holds true with $\gamma \leq 1, m \leq 3$.

Proof. See Appendix B.

All the above can be summarized in the following theorem.

Theorem 3.10 (Stability of the time-domain PML of Lemma 3.6). Let the sesquilinear form $\tilde{A}_{\sigma}(u, v)$ satisfy the conditions of Lemma 3.6. Then the system (27) with $\varepsilon_{1}(s)=b(s)^{-1}, \varepsilon_{2}(s)=a(s)^{-1}\left(1+\sigma s^{-1} \psi(s)\right)^{2}$ and $\mu(s)=c(s)$ is well-posed and stable.

The following direct corollary of Lemma 3.6 provides a simple way to construct a stable PML.

Theorem 3.11 (Construction of stable PMLs in the direction $x$ ). Let a sesquilinear form A(u,v) given by (18) be passive in the sense of Definition 2.11. Then for all $\sigma \geq 0$, the form

$$
A_{\sigma}(u, v)=a(s)\left(1+\frac{\sigma a(s)}{s}\right)^{-2}\left(\partial_{x} u, \partial_{x} v\right)+b(s)\left(\partial_{y} u, \partial_{y} v\right)+s^{2} c(s)(u, v), \quad u, v \in H^{1}\left(\mathbb{R}^{2}\right)
$$

obtained from (40) with a particular choice $\psi(s)=a(s)$, is passive in the sense of Definition 2.11.

Proof. Setting $\psi(s)=a(s)$ shows that the assumptions of Lemma 3.6 are satisfied.

Example 3.12 (Uniaxial Cold Plasma). Consider the plasma model (8), with the PML in $y$-direction chosen as $\psi(s)=\varepsilon_{1}(s)^{-1}$. The stability of this PML was confirmed in [6,7]. In this case in Corollary $3.7 m=\mu=0$.

Theorem 3.11 applied to the isotropic Drude model (6) suggests that the choice $\psi(s)=\varepsilon(s)^{-1}$ in the change of variables (39) leads to a stable PML. This is confirmed by the analysis and numerical experiments in [9]. In the same work it was demonstrated that a more general change of variables leads to a stable PML, more precisely, $\psi(s)=\left(1+\frac{\omega_{\ell}^{2}}{s^{2}}\right)^{-1}$, where $\omega_{\ell} \in\left[\min \left(\omega_{e}, \omega_{m}\right), \max \left(\omega_{e}, \omega_{m}\right)\right]$. We explain this in the following proposition.

Proposition 3.13 (Other stable PMLs for isotropic models). Let a sesquilinear form A(u,v) given by (18) with $a(s)=b(s)$ be passive in the sense of Definition 2.11. Let $\psi(s)=\left(\alpha a(s)^{-1}+(1-\alpha) c(s)\right)^{-1}, 0 \leq \alpha \leq 1$.

Then for all $\sigma \geq 0$, the form $\tilde{A}_{\sigma}(u, v)$ defined in (41) is passive.

Proof. It is sufficient to verify conditions of Lemma 3.6:

(1) thanks to (14), it is sufficient to check $\operatorname{Re}\left(s \psi(s)^{-1}\right)=\alpha \operatorname{Re}\left(s a(s)^{-1}\right)+(1-\alpha) \operatorname{Re}(s c(s))>0$.

(2) since $b(s)=a(s), \psi(s) a(s)^{-1} b(s)=\psi(s)$.

(3) to show the required bound for $a(s)^{-1} \psi(s) c(s)$, we use (14):

$$
\operatorname{Re}\left(\bar{s} \psi(s)^{-1} a(s) c(s)^{-1}\right)=\alpha \operatorname{Re}\left(\bar{s} c(s)^{-1}\right)+(1-\alpha) \operatorname{Re}(\bar{s} a(s))>0 .
$$

Finally, to construct a stable PML in corners, we extend the statement of Theorem 3.11. Recall that in a corner we perform changes of several variables:

$$
x \rightarrow x\left(1+s^{-1} \psi_{x}(s) \sigma_{x}\right), \quad y \rightarrow y\left(1+s^{-1} \psi_{y}(s) \sigma_{y}\right)
$$


Theorem 3.14 (PML stable in a corner). Let a sesquilinear form $A(u, v)$ given by (18) be passive in the sense of Definition 2.11. Then for all $\sigma_{x}, \sigma_{y} \geq 0$, the form

$A_{\sigma_{x}, \sigma_{y}}(u, v)=a(s)\left(1+\frac{\sigma_{x} a(s)}{s}\right)^{-2}\left(\partial_{x} u, \partial_{x} v\right)+b(s)\left(1+\frac{\sigma_{y} b(s)}{s}\right)^{-2}\left(\partial_{y} u, \partial_{y} v\right)+s^{2} c(s)(u, v), u, v \in H^{1}\left(\mathbb{R}^{2}\right)$,

obtained from (18) by applying the PML (46) with $\psi_{x}=a, \psi_{y}=b$, is passive in the sense of Definition 2.11.

Proof. The proof of this result follows the same arguments as the proof of Lemma 3.6.

The corresponding time-domain stability result can be formulated as in Theorem 3.10.

Remark 3.15. The stability of the PML as proved in this work implies the uniform stability of the PML as defined in [9], see also Proposition 2.18.

While the changes of variables suggested in this section lead to stable systems, it is not obvious whether the resulting layer is absorbing, i.e. that it leads to the energy decay. This question is addressed in [47].

\subsection{Numerical Verification of the Results}

Example 3.16. (The system of Example 3.2; verification of Theorem 3.11) First of all, we apply the obtained results to the system described in Example 3.2. We use exactly the same parameters, see (34) and Table 1, however, in this case we construct the PML system with the help of the PML change of variables (32) with $\psi(s)=\varepsilon_{2}(s)^{-1}$. As before, in $y$-direction we use zero Dirichlet boundary conditions.

The results of this experiment are shown in Figure 2. One clearly sees that the solution is stable. Interestingly, in this case the norm of the solution decays very lightly inside the domain and the PML. This is partially due to the dispersive, anisotropic nature of the problem (the solution remains non-zero inside a bounded domain for a fairly long time), and partially because the PML was used only in one direction (cf. e.g. Figure 3, where the PML was used in two directions). Notice that the oscillations in the norm of the solution are likely due to the dispersive behaviour of the problem.

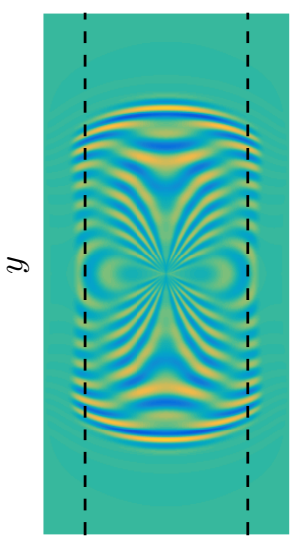

$x$

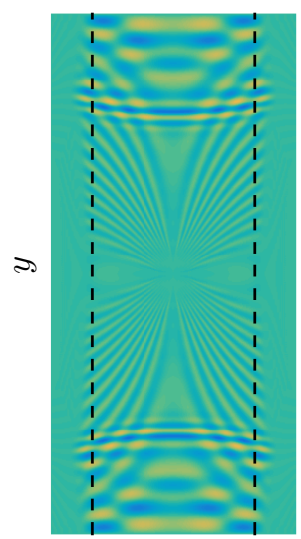

$x$

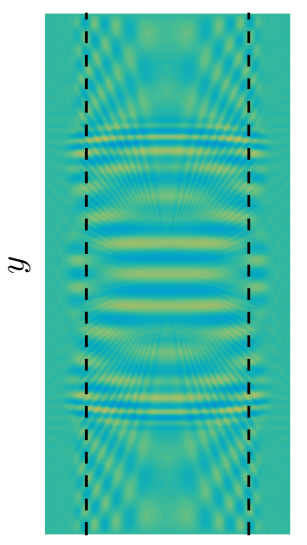

$x$

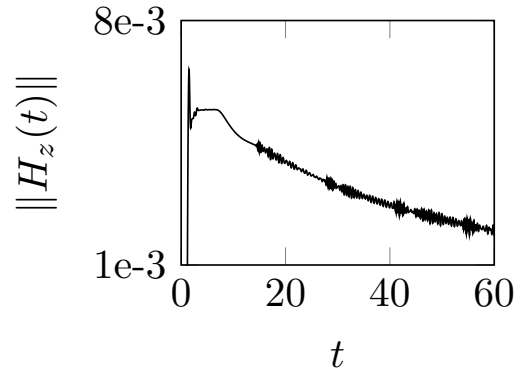

FiguRE 2. From left to right: a solution $H_{z}$ of Example 3.16 computed with the new PML at $t=10,20,35$; the dependence of the $L^{2}$-norm of $H_{z}$ with respect to time. The boundary between the domain and the layer is marked in black. 
Example 3.17. (Anisotropic Lorentz Material; verification of Theorem 3.14) We consider an anisotropic Lorentz material (9) with the parameters

$$
\varepsilon_{1}(s)=1+\frac{1}{12}\left(\frac{325}{s^{2}+4^{2}}+\frac{119}{s^{2}+8^{2}}\right), \quad \varepsilon_{2}(s)=1+\frac{16}{s^{2}+1}+\frac{16}{s^{2}+5^{2}}, \quad \mu(s)=1+\frac{3}{s^{2}+2^{2}} .
$$

Notice that the corresponding time-domain system is stable, since $a=\varepsilon_{2}^{-1}, b=\varepsilon_{1}^{-1}, c=\mu$ satisfy conditions of Definition 2.11, cf. Theorem 4.4. This time we apply the PML change of variables in both directions (46) with $\psi_{x}=\varepsilon_{2}^{-1}$ and $\psi_{y}=\varepsilon_{1}^{-1}$. The stability of such a PML is proven in Theorem 3.14.

The rest of the parameters for this experiment are provided in Table 2. The results of the experiment are

$$
\begin{array}{ccccccccr}
L_{x} & L_{y} & L_{x}^{\sigma} & L_{y}^{\sigma} & \sigma_{x}(x) & \sigma_{y}(y) & \Delta x & \Delta t & f(t, x, y) \\
\hline 8 & 8 & 2 & 2 & 20 x^{2} & 20 y^{2} & 0.025 & 0.0125 & 10(t-1.6) \mathrm{e}^{-15(t-1.6)^{2}} \mathrm{e}^{-20 x^{2}-20 y^{2}}
\end{array}
$$

TABLE 2. Parameters for the experiment with dielectric permittivity and magnetic permeability (47). The notation can be found in the beginning of Section 3.1.2.

shown in Figure 3. Notice that the classical PMLs are unstable, whereas with the use of the new PMLs the norm of the solution decays. Due to the anisotropy of the model, when using the classical PMLs, the instability in the $x$-direction is more pronounced and occurs earlier than the instability in the $y$-direction.
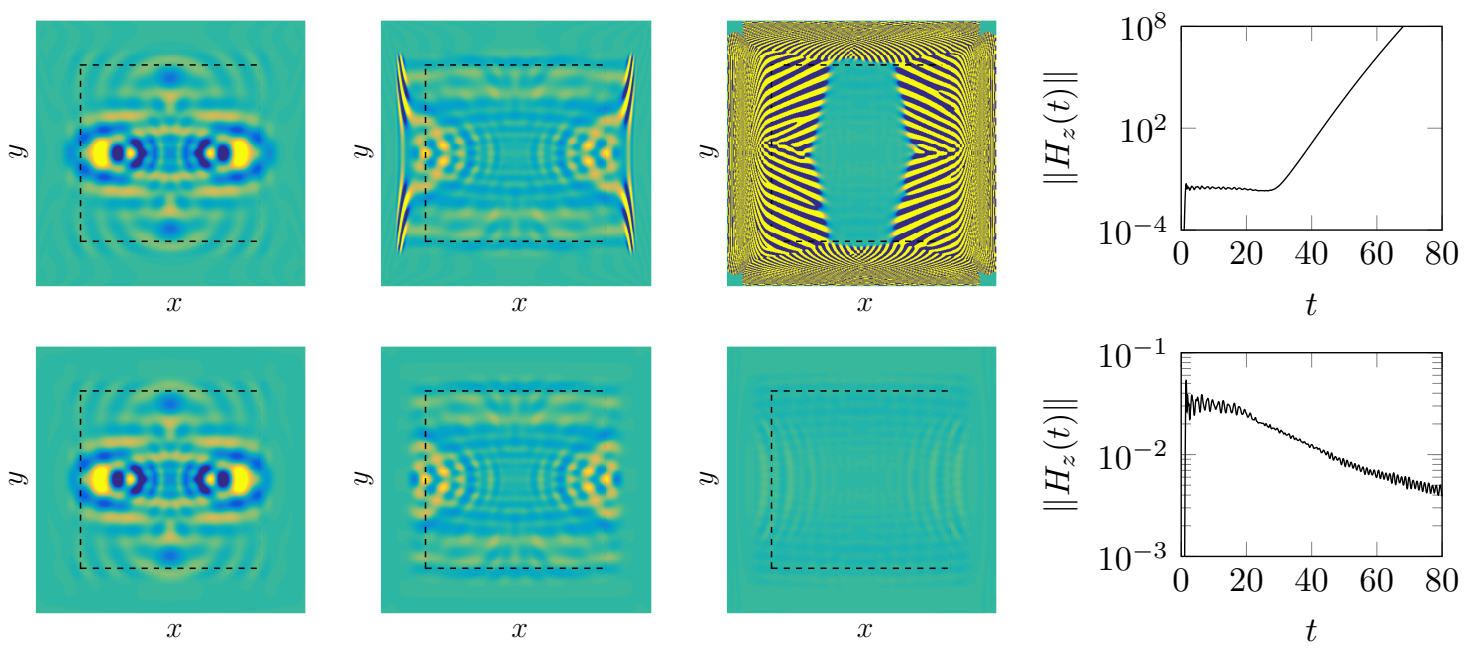

Figure 3. Top row: the experiment was done with the help of the classical PML. Bottom row: the experiment is done with the new PML. From left to right: a solution $H_{z}$ of Example 3.17 at the times $t=15,30,80$, computed with the help of corresponding PMLs; the dependence on time of the $L^{2}$-norm of this solution measured in the whole domain. The boundary between the physical domain and the PML is marked in black.

Example 3.18. (Verification of Theorem 3.11 for Materials with Losses) Let us consider the problem (5) with the parameters:

$$
\varepsilon_{1}(s)=1, \quad \varepsilon_{2}(s)=1+\frac{2}{3(s+3)}+\frac{25}{12 s}+\frac{3}{4(s+2)}, \quad \mu(s)=1 .
$$


By a direct computation one can verify that the corresponding sesquilinear form is passive. Unlike the previous two examples, this problem does not fit the framework of Lorentz materials (9). Moreover, the existing necessary PML stability condition [9] does not cover this class of models.

The parameters for this experiment are provided in Table 3. We use the PML only in the direction $x$. A

\begin{tabular}{ccccccccr}
$L_{x}$ & $L_{y}$ & $L_{x}^{\sigma}$ & $L_{y}^{\sigma}$ & $\sigma_{x}(x)$ & $\sigma_{y}(y)$ & $\Delta x$ & $\Delta t$ & $f(t, x, y)$ \\
\hline 8 & 12 & 2 & 0 & $50 x^{2}$ & 0 & 0.05 & 0.025 & $120 \mathrm{e}^{-16(t-2)^{2}} \mathrm{e}^{-300 x^{2}-800 y^{2}}$
\end{tabular}

TABLE 3. Parameters for the experiment with dielectric permittivity and magnetic permeability (48). The notation can be found in the beginning of Section 3.1.2.

numerical comparison of the new PMLs (32) with $\psi(s)=\varepsilon_{2}(s)^{-1}$ with the classical perfectly matched layers is shown in Figure 4. Contrary to the classical PMLs, the new change of variables leads to a stable system.
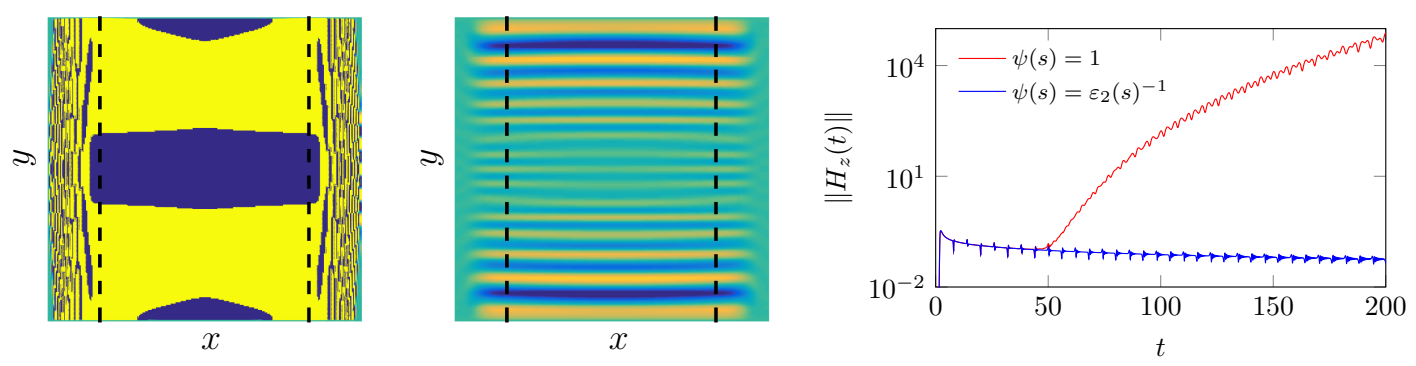

Figure 4. From left to right: a solution $H_{z}$ to the problem of Example 3.18 at $t=200$ computed with the classical PMLs; the same quantity computed with the new PMLs; the comparison of $L^{2}$-norms of the solution $H_{z}$, computed with two different PMLs, measured in the whole domain. The boundary between the physical domain and the PML is in black.

Example 3.19. (Nonlocal Materials) Another (somewhat academic) example is provided by application of a PML to a problem with non-local operators. We choose the problem parameters as:

$$
\varepsilon_{1}(s)=1, \varepsilon_{2}(s)=\left(1+s\left(1+\mathrm{e}^{-0.5 s}\right)^{-1}\right)^{-1}, \mu(s)=1 .
$$

The above functions satisfy $\operatorname{Re}(s \mu(s))>0$ and $\operatorname{Re}\left(\bar{s} \varepsilon_{j}(s)^{-1}\right)>0$, with $j=1,2$, for all $s \in \mathbb{C}_{+}$. Notice as well that $\mathcal{L}^{-1}\left(\mathrm{e}^{-0.5 s} \hat{u}(s)\right)(t)=u(t-0.5)$ for causal functions. We compare the performance of the classical PMLs employed in both directions, and the new PMLs (32) with $\psi(s)=\varepsilon_{2}(s)^{-1}$ in the direction $x$, and $\psi(s)=1$ (i.e. classical PMLs) in the direction $y$.

To semi-discretize the corresponding equation (10) in time, we employ the Runge-Kutta (3-stage Radau IIA) convolution quadrature (CQ) method [48-50], and use the FFT-based algorithm described in [51]. A similar algorithm for volumetric problems can be found in [52]. To discretize the resulting problem in space we apply second order central finite differences. Notice that for stable perfectly matched layer there is no need to employ the CFL condition restriction [52], since the method we use is implicit and $A$-stable. However, a time step restriction may be required if the PML system is unstable. The parameters of the experiment are given in Table 4. The results of the comparison of two PMLs can be found in Figure 5. We can clearly see that the solution computed with Bérenger's PML is unstable. To investigate whether the instability problem comes the discretization, we computed as well the norms of the solution to the problem computed with a twice finer time step, see Figure 5. Refining the time step even aggravates the Bérenger's PML instability, which indicates the 


\begin{tabular}{rcccccccc}
$L_{x}$ & $L_{y}$ & $L_{x}^{\sigma}$ & $L_{y}^{\sigma}$ & $\sigma_{x}(x)$ & $\sigma_{y}(y)$ & $\Delta x$ & $\Delta t$ & $f(t, x, y)$ \\
\hline 12 & 12 & 2 & 2 & $10 x^{2}$ & $10 y^{2}$ & 0.025 & 0.1 & $\mathrm{e}^{-40 x^{2}-40 y^{2}-12(t-10)^{2}}\left(24^{2}(t-10)^{2}-24\right)$
\end{tabular}

TABLE 4. Parameters for the experiment with dielectric permittivity and magnetic permeability of Example 3.19. The notation can be found in the beginning of Section 3.1.2. We discretize directly the problem (5) with the PML change of variables (32). We use zero initial conditions and the right hand side (in the time domain) $f(t, x, y)$.
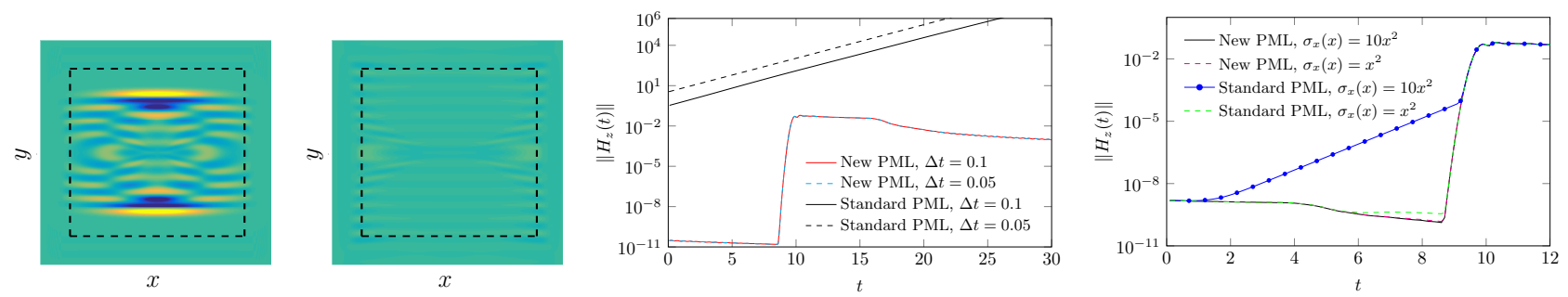

FiguRE 5. From left to right: a solution $H_{z}$ of Example 3.19 at $t=14$ and $t=20$ computed with the help of the new PML; the dependence of the $L^{2}$-norm of the solution $H_{z}$ measured in the physical domain and the PML with respect to time, for the stable and unstable PMLs, for $t \leq 30$; the dependence of the $L^{2}$-norm of the solution $H_{z}$ measured in the physical domain and the PML with respect to time, for the stable and unstable PMLs, computed for $t \leq 12$ and different absorption parameters. The boundary between the physical domain and the PML is marked in black.

convergence problem of the $\mathrm{CQ}$ in this case. However, refining the time step results in a stable, convergent solution when the new PML is used.

Indeed, the result produced by convolution quadrature discretization of the unstable PML has large stepwise errors. Moreover, the result is non-causal (in our experiments the right hand side $\|f(t, x, y)\|_{\infty}<10^{-8}$ for $t \leq 8.5$, i.e. it almost vanishes, while this is not true for the solution). Such a global behaviour may be attributed to the algorithm that we used for the implementation of the CQ, which computes the solution of (5) in the Laplace domain for many values of frequencies $s \in \mathbb{C}_{+}$, and then comes back to the time domain. As a result, the instability of the PML propagates over all the time steps, which is shown in Figure 5, where this is expressed in the non-causal behaviour of the solution. Another problem comes from the fact that the discretizations of (18), to which the classical PMLs are applied, are highly ill-conditioned (in the algorithm we use, these matrices should be inverted to compute the solution). This is likely to be caused by the fact that the corresponding matrix has some of its eigenvalues in the right complex half-plane.

We checked the first assumption (i.e. propagation of the error globally) by computing the solution with the help of the unstable PML on the interval $[0,12]$, before the instability occurs, see the left-most plot in Figure 5. It appeared that the norm of the solution on this interval did not grow exponentially, however the difference between the solutions computed with two PMLs was non-negligible. To check the second assumption (i.e. the effect of matrix ill-conditioning), we reduced the absorption parameter, by setting $\sigma_{x}(x)=x^{2}$, while keeping the rest of parameters as in Table 4 , and again computed the solution on $[0,12]$. This allowed to reduce the condition number of the matrices. The results displayed in the leftmost plot of Figure 5 show that the solutions computed with stable and unstable PMLs on the interval $[0,12]$ almost coincide.

Summarizing all said, our experiments demonstrate that the solution remains stable when the new perfectly matched layer is applied, however, there are convergence and stability problems when the standard PMLs used. Let us also remark that in general the discretization by the Runge-Kutta method Radau IIA introduces some 
dissipation into the problem (which can be controlled by decreasing the time step), however, even in this case we observe the instability of the classical PMLs.

\section{Dispersive Systems with Rational Dielectric Permittivity and Magnetic PERMEABILITY}

In the previous section we have shown how to construct a stable PML for a model described by a passive sesquilinear form (18). In this section we will study a special case when the coefficients of the sesquilinear form are rational even functions (however, most of the results are valid as well for meromorphic even functions). This section is organized as follows. First of all, we will discuss some important properties of rational functions under consideration. Next, we will reformulate the corresponding systems, with the PML and without the PML, in the time domain.

\subsection{Properties of Even Rational Functions which Satisfy the Passivity Condition}

We will consider a set of rational functions $r(z)$ satisfying the following assumptions (these are the same assumptions as in the work [9]).

Assumption 4.1. (1) $r(z)$ can be represented as $r(z)=1+\frac{p\left(z^{2}\right)}{q\left(z^{2}\right)}$, where $p(z)$ and $q(z)$ are polynomials with real coefficients that have no common roots, s.t. $\operatorname{deg} p<\operatorname{deg} q$.

(2) all zeros and poles of $r(z)$ lie on the imaginary axis.

Recall that given $r(z), z \in \mathbb{C}_{+}$, by $\tilde{r}(\omega)$ we denote the restriction $\tilde{r}(\omega)=r(-i \omega), \operatorname{Im} \omega \geq 0$, see $(3)$.

Remark 4.2 (Physical Relevance). Later on we will assume that $\varepsilon_{j}(s)$ for $j=1,2$, and $\mu(s)$ in (5) belong to the above class. These requiremends are indeed physically meaningful:

(1) requiring that $\underline{\underline{\varepsilon}}$ and $\mu$ are rational functions is equivalent to the fact that in the time domain the system (5) can be written with the help of local (i.e. linear combinations of $\partial_{t}^{(k)}$, for $k \in \mathbb{N}_{0}$ ) operators;

(2) $\underline{\underline{\tilde{\varepsilon}}}(\omega), \tilde{\mu}(\omega) \rightarrow 1$ as $\omega \rightarrow \infty$ is a requirement that can be found in [53, §59, p. 251]; this means that for large frequencies the system becomes non-dispersive;

(3) in a so-called transparency regime [53, §64, p. 260] the rational functions $\underline{\underline{\varepsilon}}(s), \mu(s)$ are real on the imaginary axis. Moreover, in this case $\underline{\underline{\tilde{\varepsilon}}}, \tilde{\mu}$ are even functions of $\omega$ (see [53, p. 250]). From this it follows that these rational functions should have real coefficients.

Let us remark that many of the results of this section are known, or follow from known theorems; however, we were not able to find a single reference that would summarize all the properties we will make use of later. Some of the results that we describe in this section are summarized as well in [24].

We can formulate the following a priori property of passive functions.

Lemma 4.3. Let $r(s)$ be a passive function satisfying Assumption 4.1. Then all of its poles and zeros are real.

Proof. The passivity requirement implies that $r(s)$ cannot have poles or zeros in $\mathbb{C}_{+}$. Thanks to the fact that $r(s)$ is even, all its poles and zeros lie on the imaginary axis.

The following result describes rational functions that satisfy the passivity requirement. It can be obtained from [27, Corollary 10.1], combined with [26, Theorem 5]. 
Theorem 4.4 ( [27], [26]). Let $r(s)$ be a rational function satisfying Assumption 4.1. Then this function is passive, i.e. $\operatorname{Re}(\operatorname{sr}(s))>0, s \in \mathbb{C}_{+}$, if and only if

$$
r(s)=1+\sum_{\ell=0}^{n} \frac{r_{\ell}}{s^{2}+\omega_{\ell}^{2}}, \quad r_{\ell}>0, \omega_{\ell} \in \mathbb{R}, \ell=0, \ldots, n
$$

Moreover, in this case

$$
\operatorname{Re}(s r(s))>\operatorname{Re} s
$$

Proof. Let us show that any passive function satisfying Assumption 4.1 satisfies (50). From Assumption 4.1 it follows that all the poles of $r(s)$ lie on the imaginary axis. Applying the first part of Lemma 2.5 to $s r(s)$, which shows that all purely imaginary poles of $r(s)$ are simple, and zero may be a pole of multiplicity either 1 or 2 , and using the fact that $r(s)$ is even, we obtain the following partial fraction expansion for $r(s)$ :

$$
r(s)=1+\frac{r_{0}}{s^{2}}+\sum_{\ell=1}^{n} \frac{\mu_{\ell}^{+}}{s+i \omega_{\ell}}+\sum_{\ell=1}^{n} \frac{\mu_{\ell}^{-}}{s-i \omega_{\ell}}, \quad \omega_{\ell}>0, \ell=1, \ldots, n .
$$

Now, since $r(s)=r(-s)$ in $\mathbb{C}$, we obtain that $\frac{\mu_{\ell}^{+}}{s+i \omega_{\ell}}+\frac{\mu_{\ell}^{-}}{s-i \omega_{\ell}}=\frac{\mu_{\ell}^{+}}{-s+i \omega_{\ell}}+\frac{\mu_{\ell}^{-}}{-s-i \omega_{\ell}}$, for all $\ell=1, \ldots, n$, and thus $\mu_{\ell}^{+}=-\mu_{\ell}^{-}$for all $\ell=1, \ldots, n$. The positivity of residues of $\operatorname{sr}(s)$, see Lemma 2.5 , gives

$$
r_{0}>0,-i \omega_{\ell} \mu_{\ell}^{+}>0, \quad \ell=1, \ldots, n .
$$

From this it immediately follows that $\mu_{\ell}^{+} \in i \mathbb{R}_{>0}$. This allows us to obtain the expansion $r(s)=1+\sum_{\ell=0}^{n} \frac{r_{\ell}}{s^{2}+\omega_{\ell}^{2}}$, with $\omega_{\ell} \geq 0$ for $\ell=0, \ldots, n$, where $r_{\ell}=-2 i \omega_{\ell} \mu_{\ell}^{+}$, with $\ell=1, \ldots, n$. The positivity of $r_{\ell}$ follows from (53).

Now let us demonstrate that $r(s)$ given by (50) is passive, by showing that (51) holds true in this case:

$$
\operatorname{Re}(s r(s))=\operatorname{Re} s+\operatorname{Re}\left(\sum_{\ell=0}^{n} \frac{r_{\ell} s}{s^{2}+\omega_{\ell}^{2}}\right)=\operatorname{Re} s+\operatorname{Re}\left(\sum_{\ell=0}^{n} \frac{r_{\ell} s\left(\bar{s}^{2}+\omega_{\ell}^{2}\right)}{\left|s^{2}+\omega_{\ell}^{2}\right|^{2}}\right)>\operatorname{Re} s,
$$

when $s \in \mathbb{C}_{+}$.

This result shows in particular that under Assumption 4.1 the passivity requirement can be satisfied only by the Lorentz materials (9). Naturally, we would also like to understand what the reciprocals of rational functions satisfying the passivity requirement look like.

Theorem 4.5. Let $r(s)$ be a rational function satisfying Assumption 4.1. Then $\operatorname{Re}(\bar{s} r(s))>0, s \in \mathbb{C}_{+}$, if and only if

$$
r(s)=1-\sum_{\ell=0}^{n} \frac{r_{\ell}}{s^{2}+\omega_{\ell}^{2}}, \quad r_{\ell}>0, \omega_{\ell} \in \mathbb{R} \backslash\{0\}, \ell=0, \ldots, n, \quad r(0)=1-\sum_{\ell=0}^{n} \frac{r_{\ell}}{\omega_{\ell}^{2}} \geq 0 .
$$

Moreover,

$$
\operatorname{Re}(\bar{s} r(s)) \geq r(0) \operatorname{Re} s+C \min \left(1,(\operatorname{Re} s)^{2}\right) \operatorname{Re} s, \quad C>0 .
$$

Proof. First let us show that any rational function satisfying Assumption 4.1 and $\operatorname{Re}(\bar{s} r(s))>0$, when $s \in \mathbb{C}_{+}$, is of the form (54). 
Thanks to Lemma 2.5, which says that $s^{-1} r(s)$ can have a simple pole in zero only, $r(s)$ cannot have a pole in zero; moreover, all the poles of $r(s)$ are imaginary, due to Assumption 4.1, and thus, due to Lemma 2.5, are simple. Like in Theorem 4.4, we write

$$
r(s)=1+\sum_{\ell=0}^{n} \frac{\mu_{\ell}^{+}}{s+i \omega_{\ell}}+\sum_{\ell=0}^{n} \frac{\mu_{\ell}^{-}}{s-i \omega_{\ell}}, \quad \omega_{\ell}>0, \ell=1, \ldots, n .
$$

The same argument as in the proof of Theorem 4.4, shows that $\mu_{\ell}^{+}=-\mu_{\ell}^{-}$, and $r(s)=1-\sum_{\ell=0}^{n} \frac{r_{\ell}}{s^{2}+\omega_{\ell}^{2}}$, where $\omega_{\ell}>0, \ell=0, \ldots, n$, and $r_{\ell}=2 i \omega_{\ell} \mu_{\ell}^{+}>0$. Lemma 2.5 says that residues of $s^{-1} r(s)$ in all poles should be positive:

$$
\lim _{s \rightarrow i \omega_{\ell}}\left(s-i \omega_{\ell}\right) s^{-1} r(s)=\frac{r_{\ell}}{2 \omega_{\ell}^{2}}>0 .
$$

If $r(0) \neq 0$, the positivity of the residue of $s^{-1} r(s)$ in zero implies that $r(0)>0$.

To show that any $r(s)$ of the form (54) satisfies $\operatorname{Re}(\bar{s} r(s))>0$ in $\mathbb{C}_{+}$, we will demonstrate the validity of the bound (55). First of all,

$$
r(s)=1-\sum_{\ell=0}^{n} \frac{r_{\ell}}{s^{2}+\omega_{\ell}^{2}}=1-\sum_{\ell=0}^{n} \frac{r_{\ell}}{\omega_{\ell}^{2}}+\sum_{\ell=0}^{n} \frac{r_{\ell}}{\omega_{\ell}^{2}}-\sum_{\ell=0}^{n} \frac{r_{\ell}}{s^{2}+\omega_{\ell}^{2}}=r(0)+\sum_{\ell=0}^{n} \frac{r_{\ell} s^{2}}{\omega_{\ell}^{2}\left(s^{2}+\omega_{\ell}^{2}\right)}
$$

Hence, for all $s \in \mathbb{C}_{+}$it holds:

$$
\begin{aligned}
\operatorname{Re}(\bar{s} r(s)) & =r(0) \operatorname{Re} s+\operatorname{Re} \sum_{\ell=0}^{n} \frac{r_{\ell}|s|^{2} s\left(\bar{s}^{2}+\omega_{\ell}^{2}\right)}{\omega_{\ell}^{2}\left|s^{2}+\omega_{\ell}^{2}\right|^{2}}=r(0) \operatorname{Re} s+\operatorname{Re} s \sum_{\ell=0}^{n} r_{\ell} \omega_{\ell}^{-2}|s|^{2}\left(|s|^{2}+\omega_{\ell}^{2}\right)\left|s^{2}+\omega_{\ell}^{2}\right|^{-2} \\
& \geq r(0) \operatorname{Re} s+\operatorname{Re} s \sum_{\ell=0}^{n} r_{\ell} \omega_{\ell}^{-2}|s|^{2}\left(|s|^{2}+\omega_{\ell}^{2}\right)^{-1}
\end{aligned}
$$

For any $\omega_{\ell} \in \mathbb{R}^{*}$, the function $f(x)=\frac{x^{2}}{x^{2}+\omega_{\ell}^{2}}=1-\frac{\omega_{\ell}^{2}}{x^{2}+\omega_{\ell}^{2}}$ grows in $x$ for $x>0$. Since $0<\operatorname{Re} s<|s|$, we obtain

$$
\frac{|s|^{2}}{|s|^{2}+\omega_{\ell}^{2}} \geq \frac{(\operatorname{Re} s)^{2}}{(\operatorname{Re} s)^{2}+\omega_{\ell}^{2}} \geq \frac{(\operatorname{Re} s)^{2}}{2 \max \left((\operatorname{Re} s)^{2}, \omega_{\ell}^{2}\right)}=\frac{1}{2}(\operatorname{Re} s)^{2} \min \left((\operatorname{Re} s)^{-2}, \omega_{\ell}^{-2}\right) \geq \mathrm{C} \min \left(1,(\operatorname{Re} s)^{2}\right),
$$

for some $C>0$. This, combined with (57), gives (55).

Another property of passive materials, which is exploited in [9], is the so-called growing property. More precisely, a rational function $r(s)$ satisfies the growing property if for all $\omega \in \mathbb{R}$ except for poles of $\tilde{r}(\omega)$, it holds $(\omega \tilde{r}(\omega))^{\prime}>0$. It is interesting that in physical literature [54], [53, p.256] the above property is shown to be crucial for the positivity of the energy density.

We provide a minor generalization of this statement in the following two lemmas, stating before an auxiliary result that can be found in [26, Theorem V, Coroll. 1].

Lemma 4.6 ([26]). Let $r(s)$ satisfy Assumption 4.1. Let $g_{1}(s)=s r(s)$, and $g_{2}(s)=s^{-1} r(s), s \in \mathbb{C}_{+}$. If all zeros of $g_{\alpha}(s)$ are simple, and the derivative $\frac{d}{d s} g_{\alpha}(s)$ is real and positive in these zeros, then $\operatorname{Re}\left(g_{\alpha}(s)\right)>0$ for all $s \in \mathbb{C}_{+}$. Here $\alpha=1$ or $\alpha=2$.

To prove the validity of the statement, we start with the following trivial result.

Lemma 4.7. Let $r(s)$ satisfy Assumption 4.1. Then: 
(1) if all the poles of $\operatorname{sr}(s)$ are simple, and the corresponding residues are positive, then $\operatorname{Re}(\operatorname{sr}(s))>0, s \in$ $\mathbb{C}_{+}$.

(2) if all the poles of $s^{-1} r(s)$ are simple, and the corresponding residues are positive, then $\operatorname{Re}(\bar{s} r(s))>$ $0, s \in \mathbb{C}_{+}$.

Proof. The result can be proved following the lines of Theorems 4.4 and 4.5. More precisely, in the first case $r(s)$ is of the form (52); the positivity of residues and evenness of the function $r(s)$ results in the expansion (50), which, in turn, shows that $r(s)$ is passive, see Theorem 4.4.

Now we can give a proof of Lemma 4.6.

Proof. Let $\alpha=1$ or 2 . Notice that if all the zeros of $g_{\alpha}(s)$ are simple, and the derivative $\frac{d}{d s} g_{\alpha}(s)$ is real and positive in these zeros, then all the poles of $f_{\alpha}(s)=g_{\alpha}(s)^{-1}$ are simple, and the residues of $f_{\alpha}(s)$ in these poles are positive:

$$
\operatorname{Res}_{s=s_{0}} f_{\alpha}(s)=\lim _{s \rightarrow s_{0}}\left(s-s_{0}\right) f_{\alpha}(s)=\lim _{s \rightarrow s_{0}}\left(s-s_{0}\right) g_{\alpha}(s)^{-1}=\lim _{s \rightarrow s_{0}} \frac{s-s_{0}}{g_{\alpha}(s)-g_{\alpha}\left(s_{0}\right)}=\left(g_{\alpha}^{\prime}\left(s_{0}\right)\right)^{-1}>0 .
$$

Combined with Lemma 4.7, we obtain the desired result.

Finally, let us discuss the equivalence of the growing property and passivity.

Lemma 4.8. Let $r(s)$ satisfy Assumption 4.1. Then the following conditions are equivalent:

(M1) $(\omega \tilde{r}(\omega))^{\prime}>0$ in all points but poles of $\tilde{r}(\omega)$;

(M2) the function $r(s)$ satisfies $\operatorname{Re}(\operatorname{sr}(s))>0, s \in \mathbb{C}_{+}$.

Proof. Thanks to Lemma 4.6, to show that (M1) implies (M2), it is sufficient to verify that from (M1) it follows that the zeros of $R(s)=s r(s)$ are simple, and $R^{\prime}(s)>0$ in these zeros. Notice that $\tilde{R}(\omega)=-i \omega \tilde{r}(\omega)$.

First let us show the implication (M1) $\Longrightarrow(\mathrm{M} 2)$. In zeros $s=-i \omega_{0}$ of $\operatorname{sr}(s)$

$$
\left.\frac{d R}{d s}\right|_{s=-i \omega_{0}}=r\left(-i \omega_{0}\right)-i \omega_{0} r^{\prime}\left(-i \omega_{0}\right)=\tilde{r}\left(\omega_{0}\right)+\tilde{r}^{\prime}\left(\omega_{0}\right) \omega_{0}=\left.(\omega \tilde{r}(\omega))^{\prime}\right|_{\omega=\omega_{0}}>0
$$

from which we obtain as well the simplicity of zeros of $R(s)$.

Notice that (M2) is equivalent to requiring that $\tilde{r}(\omega)=1-\sum_{\ell=0}^{n} \frac{r_{\ell}}{\omega^{2}-\omega_{\ell}^{2}}, r_{\ell}>0, \omega_{\ell} \in \mathbb{R}, \ell=0, \ldots, n$, see Theorem 4.4. Thus, (M2) $\Longrightarrow$ (M1) follows by a direct computation:

$$
(\omega \tilde{r}(\omega))^{\prime}=1+\sum_{\ell=0}^{n} r_{\ell}\left(\frac{2 \omega^{2}}{\left(\omega^{2}-\omega_{\ell}^{2}\right)^{2}}-\frac{1}{\omega^{2}-\omega_{\ell}^{2}}\right)=1+\sum_{\ell=0}^{n} r_{\ell} \frac{\omega^{2}+\omega_{\ell}^{2}}{\left(\omega^{2}-\omega_{\ell}^{2}\right)^{2}}>0
$$

in all points but poles of $\tilde{r}(\omega)$.

A similar result can be formulated for functions $r(s)$, which satisfy $\operatorname{Re}(\bar{s} r(s))>0$ in $\mathbb{C}_{+}$.

Lemma 4.9. Let $r(s)$ satisfy Assumption 4.1. Then the following conditions are equivalent:

(M1) $\left(\omega^{-1} \tilde{r}(\omega)\right)^{\prime}<0$ in all points $\omega \in \mathbb{R}$ but poles of $\omega^{-1} \tilde{r}(\omega)$;

(M2) the function $r(s)$ satisfies $\operatorname{Re}(\bar{s} r(s))>0, s \in \mathbb{C}_{+}$.

Proof. Thanks to Lemma 4.6, to show that (M1) implies (M2), it is sufficient to verify that from (M1) it follows that the zeros of $R(s)=s^{-1} r(s)$ are simple, and $R^{\prime}(s)>0$ in these zeros. Notice that $\tilde{R}(\omega)=i \omega^{-1} \tilde{r}(\omega)$. First let us show the implication (M1) $\Longrightarrow(\mathrm{M} 2)$. In zeros $s=-i \omega_{0}$ of $s^{-1} r(s)$

$$
\left.\frac{d R}{d s}\right|_{s=-i \omega_{0}}=\omega_{0}^{-2} r\left(-i \omega_{0}\right)-\left(i \omega_{0}\right)^{-1} r^{\prime}\left(-i \omega_{0}\right)=\tilde{r}\left(\omega_{0}\right) \omega_{0}^{-2}-\tilde{r}^{\prime}\left(\omega_{0}\right) \omega_{0}^{-1}=-\left.\left(\omega^{-1} \tilde{r}(\omega)\right)\right|_{\omega=\omega_{0}}>0
$$


from which we obtain as well the simplicity of zeros of $R(s)$.

Notice that (M2) is equivalent to requiring that $\tilde{r}(\omega)=r(0)+\sum_{\ell=0}^{n} \frac{r_{\ell} \omega^{2}}{\omega_{\ell}^{2}\left(\omega^{2}-\omega_{\ell}^{2}\right)}, r_{\ell}>0, \omega_{\ell} \in \mathbb{R} \backslash\{0\}, \ell=0, \ldots, n$, and $r(0) \geq 0$, see (56) in Theorem 4.5. Thus, (M2) $\Longrightarrow$ (M1) follows by a direct computation:

$$
\left(\omega^{-1} \tilde{r}(\omega)\right)^{\prime}=-\frac{r(0)}{\omega^{2}}+\sum_{\ell=0}^{n} \frac{r_{\ell}}{\omega_{\ell}^{2}\left(\omega^{2}-\omega_{\ell}^{2}\right)}-\sum_{\ell=0}^{n} \frac{2 r_{\ell} \omega^{2}}{\omega_{\ell}^{2}\left(\omega^{2}-\omega_{\ell}^{2}\right)^{2}}=-\frac{r(0)}{\omega^{2}}-\sum_{\ell=0}^{n} \frac{r_{\ell}}{\omega_{\ell}^{2}} \frac{\left(\omega^{2}+\omega_{\ell}^{2}\right)}{\left(\omega^{2}-\omega_{\ell}^{2}\right)^{2}}
$$

The above expression is strictly negative in $\omega$ s.t. $\omega \neq 0$ and $\omega$ is not a pole of $\tilde{r}(\omega)$. If $r(0)=0$, the above expression is strictly negative in $\omega=0$.

The following statement is at least partially well known, cf. e.g. [28, p.308, Theorem 1].

Lemma 4.10. Let $r(s)$ satisfy Assumption 4.1. Let $\left(\omega_{\ell}\right)_{\ell=1}^{n}$ be non-negative poles of $\tilde{r}(\omega)$ ordered in ascending order, and $\left(r_{\ell}\right)_{\ell=1}^{n}$ be its non-negative zeros, ordered in ascending order (counting multiplicities). Then

(I1) $r(s)$ is passive in the sense of Definition 2.1 if and only if $0 \leq \omega_{1}<r_{1}<\omega_{2}<r_{2}<\ldots<\omega_{n}<r_{n}$.

(I2) $r(s)^{-1}$ is passive in the sense of Definition 2.1 if and only if $0 \leq r_{1}<\omega_{1}<r_{2}<\omega_{2}<\ldots<r_{n}<\omega_{n}$.

Proof. We explain the proof for (I1). The statement (I2) can be proved similarly.

Let us explain first" $\Longrightarrow$ "-part. The fact that zeros and poles of $r(s)$ are simple, with the exception of $s=0$, and the condition on the multiplicity of the pole/zero in $s=0$ follows from Lemmas 2.5. They interlace due to the same lemma (one notices that $h(s)=\operatorname{sr}(s)$ satisfies $h(-\bar{s})=-\bar{s} r(\bar{s})=\overline{h(s)}$ ).

Finally, if $\omega_{1} \neq 0$, then, necessarily $r(0)>0$. Hence if $r_{1}$ were smaller than $\omega_{1}$, the sign change in $r_{1}$ would be from the positive to negative, and the function would have to decay on $\left(0, \omega_{1}\right)$. This is a contradiction to Lemma 4.8.

If $\omega_{1}=0$, the statement follows directly from the interlacing property of poles and roots.

The same argument applies to the case $\operatorname{Re}(\bar{s} r(s))>0, s \in \mathbb{C}_{+}$.

Let us now show that the interlacing of roots and poles implies $\operatorname{Re}(\operatorname{sr}(s))>0, s \in \mathbb{C}_{+}$. We will simply make use of Lemma 4.8 .

First of all from the condition of the lemma we see that all zeros (and poles) of $\operatorname{sr}(s)$ are simple. Next, we must verify whether the function $\omega \tilde{r}(\omega)$ grows.

We start by showing that $\tilde{r}(0)>0$ or $\tilde{r}(\omega) \rightarrow-\infty$, when $\omega \rightarrow 0$. First notice that

$$
\tilde{r}(\omega)=\mathrm{C} \frac{\left(\omega^{2}-r_{1}^{2}\right)\left(\omega^{2}-r_{2}^{2}\right) \ldots\left(\omega^{2}-r_{n}^{2}\right)}{\left(\omega^{2}-\omega_{1}^{2}\right)\left(\omega^{2}-\omega_{2}^{2}\right) \ldots\left(\omega^{2}-\omega_{n}^{2}\right)}
$$

where $C=1$ due to $\tilde{r}(\omega) \rightarrow 1$ as $\omega \rightarrow \infty$. If $\omega_{1} \neq 0, \tilde{r}(0)=\left(\prod_{k=1}^{n}\left(-r_{k}^{2}\right)\right)\left(\prod_{k=1}^{n}\left(-\omega_{k}^{2}\right)\right)>0$. If $\omega_{1}=0$,

$$
\tilde{r}(\varepsilon)=\frac{\left(\varepsilon^{2}-r_{1}^{2}\right)\left(\varepsilon^{2}-r_{2}^{2}\right) \ldots\left(\varepsilon^{2}-r_{n}^{2}\right)}{\varepsilon^{2}\left(\varepsilon^{2}-\omega_{2}^{2}\right) \ldots\left(\varepsilon^{2}-\omega_{n}^{2}\right)}=\frac{(-1)^{n}\left(r_{1}^{2}-\varepsilon^{2}\right)\left(r_{2}^{2}-\varepsilon^{2}\right) \ldots\left(r_{n}^{2}-\varepsilon^{2}\right)}{\varepsilon^{2}(-1)^{n-1}\left(\omega_{2}^{2}-\varepsilon^{2}\right) \ldots\left(\omega_{n}^{2}-\varepsilon^{2}\right)} \rightarrow-\infty, \text { as } \varepsilon \rightarrow 0 .
$$

Consider two cases:

- $\tilde{r}(\omega)>0$. In this case $\omega=0$ is a zero of $\omega \tilde{r}(\omega)$. On the interval $\left(0, \omega_{1}\right)$ the function grows (otherwise it would have to intersect the real axis, which is a contradiction to $\left.r_{1}>\omega_{1}\right)$; the same is valid on the interval $\left(-\omega_{1}, 0\right)$, due to evenness of $r(s)$. Therefore, $(\omega \tilde{r}(\omega))^{\prime}>0$ on this interval. In $\omega_{1}$ the function $\tilde{r}(\omega)$ changes the sign from positive to negative, and in $r_{1}$ from negative to positive. Thanks to the interlacing of roots and poles, $(\omega \tilde{r}(\omega))^{\prime}>0$ for $\mathbb{R}$.

- $\tilde{r}(\omega)=-\infty$. Since $r_{1}<\omega_{2}$, the function $\omega \tilde{r}(\omega)$ changes its sign in $r_{1}$ from negative to positive. The same argument as before allows to conclude that $(\omega \tilde{r}(\omega))^{\prime}>0$ on $\mathbb{R}$.

Similarly we can prove the second equivalence. 


\subsection{PMLs for Anisotropic Lorentz Materials: Time Domain Formulation}

In the previous section we showed that provided that the dielectric permittivity and magnetic permeability satisfy the conditions of passivity and Assumption 4.1, the corresponding materials are Lorentz (9). This class of models can be expressed in the time domain as a system of partial differential equations coupled with ordinary differential equations. The goal of this section is to present the corresponding formulation, as well as provide a PML system obtained after the change of variables (32), which can be used in practical calculations.

\subsubsection{Time Domain System without the PML}

Recall that the Lorentz dielectric permittivity and magnetic permeability are given by (9). To rewrite the Maxwell system (2) in the time domain, we first notice that one of the ways to express the relation $s \hat{D}_{x}=s \varepsilon_{1}(s) \hat{E}_{x}$ in the time domain is via the introduction of auxiliary unknowns:

$$
\begin{aligned}
& \partial_{t} D_{x}=\partial_{t} E_{x}+\sum_{\ell=0}^{n_{x}} \varepsilon_{x \ell} j_{x \ell}, \\
& \partial_{t} j_{x \ell}+\omega_{x \ell}^{2} p_{x \ell}=E_{x}, \quad \partial_{t} p_{x \ell}=j_{x \ell}, \quad \ell=0, \ldots, n_{x} .
\end{aligned}
$$

One can verify that in the frequency domain it holds $s \hat{D}_{x}=s \varepsilon_{1}(s) \hat{E}_{x}$, provided the initial conditions $\left.D_{x}\right|_{t=0}=$ $\left.E_{x}\right|_{t=0}$, and zero initial conditions for the unknowns $j_{x \ell}$ and $p_{x \ell}, \ell=0, \ldots, n_{x}$. Similarly we deal with $s \hat{B}_{z}=$ $s \mu(s) H_{z}$. Then the Maxwell system (2) can be rewritten in the following form:

$$
\begin{aligned}
& \partial_{t} E_{x}+\sum_{\ell=0}^{n_{x}} \varepsilon_{x \ell} j_{x \ell}=\partial_{y} H_{z} \\
& \partial_{t} E_{y}+\sum_{\ell=0}^{n_{y}} \varepsilon_{y \ell} j_{y \ell}=-\partial_{x} H_{z} \\
& \partial_{t} H_{z}+\sum_{\ell=0}^{n_{\mu}} \mu_{\ell} j_{\mu \ell}=\partial_{y} E_{x}-\partial_{x} E_{y} \\
& \partial_{t} j_{m \ell}+\omega_{m \ell}^{2} p_{m \ell}=E_{m}, \quad \partial_{t} p_{m \ell}=j_{m \ell}, \ell=0, \ldots, n_{m}, m \in\{x, y\}, \\
& \partial_{t} j_{\mu \ell}+\omega_{\mu \ell}^{2} p_{\mu \ell} \quad=H_{z}, \quad \partial_{t} p_{\mu \ell}=j_{\mu \ell}, \ell=0, \ldots, n_{\mu} .
\end{aligned}
$$

\subsubsection{Time-Domain System with the PML}

Our goal is to rewrite the system (60a-60e) in the time domain with the PML change of variables (32) and non-constant $\sigma(x) \geq 0$. Let us limit our discussion to the functions $\psi(s)$ which satisfy Assumption 4.1. Additionally, let us assume that $\psi(s)^{-1}$ is of the following form (this is clarified in Theorem 5.8):

$$
\psi(s)^{-1}=1+\sum_{\ell=0}^{n_{\psi}} \frac{c_{\psi \ell}}{s^{2}+r_{\psi \ell}^{2}}, \quad r_{\psi \ell} \in \mathbb{R}, c_{\psi \ell} \in \mathbb{R}, \ell=0, \ldots, n_{\psi}
$$

Recall that (32) amounts to substituting all $\partial_{x}$ by $\left(1+\frac{\sigma \psi}{s}\right)^{-1} \partial_{x}$ in the frequency-domain formulation of (60a$60 \mathrm{e})$. For example, consider the equation for $E_{y}$ of $(60 \mathrm{a}-60 \mathrm{e})$ in the Laplace domain, with the PML (32):

$$
s \hat{E}_{y}+\sum_{\ell=0}^{n_{y}} \varepsilon_{y \ell} \hat{J}_{y \ell}=-\left(1+\frac{\sigma \psi(s)}{s}\right)^{-1} \partial_{x} \hat{H}_{z}=-\frac{s}{s+\sigma \psi} \partial_{x} \hat{H}_{z}=-\left(1-\frac{\sigma}{s \psi^{-1}+\sigma}\right) \partial_{x} \hat{H}_{z} .
$$


Thus, let us define $\hat{E}_{y}^{*}=-\left(s \psi^{-1}+\sigma\right)^{-1} \partial_{x} \hat{H}_{z}$ :

$$
\begin{aligned}
& s \hat{E}_{y}^{*}+\sum_{\ell=0}^{n_{\psi}} c_{\psi \ell} \hat{\jmath}_{E_{y}, \ell}^{*}+\sigma(x) \hat{E}_{y}^{*}=-\partial_{x} \hat{H}_{z}, \\
& s \hat{\jmath}_{E_{y}, \ell}^{*}+r_{\psi \ell}^{2} \hat{p}_{E_{y}, \ell}^{*}=\hat{E}_{y}^{*}, \quad s \hat{p}_{E_{y}, \ell}^{*}=\hat{\jmath}_{E_{y}, \ell}^{*}, \quad \ell=0, \ldots, n_{\psi} .
\end{aligned}
$$

Using the above strategy to derive all the remaining equations with the PML, the system (60a-60e) with the change of variables (32) becomes in the time domain:

$$
\begin{array}{ll}
\partial_{t} E_{x}+\sum_{\ell=0}^{n_{x}} \varepsilon_{x \ell} j_{x \ell}=\partial_{y} H_{z}, & \\
\partial_{t} E_{y}+\sum_{\ell=0}^{n_{y}} \varepsilon_{y \ell} j_{y \ell}=-\partial_{x} H_{z}-\sigma(x) E_{y}^{*}, & \\
\partial_{t} H_{z}+\sum_{\ell=0}^{n_{\mu}} \mu_{\ell} j_{\mu \ell}=\partial_{y} E_{x}-\partial_{x} E_{y}-\sigma(x) H_{z}^{*}, & \\
\partial_{t} j_{m \ell}+\omega_{m \ell}^{2} p_{m \ell}=E_{m}, & \partial_{t} p_{m \ell}=j_{m \ell}, \quad \ell=0, \ldots, n_{m}, m \in\{x, y\}, \\
\partial_{t} j_{\mu \ell}+\omega_{\mu \ell}^{2} p_{\mu \ell}=H_{z}, & \partial_{t} p_{\mu \ell}=j_{\mu \ell}, \quad \ell=0, \ldots, n_{\mu},
\end{array}
$$

coupled with the system for the auxiliary unknowns

$$
\begin{array}{ll}
\partial_{t} E_{y}^{*}+\sum_{\ell=0}^{n_{\psi}} c_{\psi \ell} j_{E_{y}, \ell}^{*}+\sigma(x) E_{y}^{*}=-\partial_{x} H_{z}, & \\
\partial_{t} j_{E_{y}, \ell}^{*}+r_{\psi \ell}^{2} p_{E_{y}, \ell}^{*}=E_{y}^{*}, & \partial_{t} p_{E_{y}, \ell}^{*}=j_{E_{y}, \ell}^{*}, \quad \ell=0, \ldots, n_{\psi}, \\
\partial_{t} H_{z}^{*}+\sum_{\ell=0}^{n_{\psi}} c_{\psi \ell \ell} j_{H_{z}, \ell}^{*}+\sigma(x) H_{z}^{*}=-\partial_{x} E_{y}, & \\
\partial_{t} j_{H_{z}, \ell}^{*}+r_{\psi \ell}^{2} p_{H_{z}, \ell}^{*}=H_{z}^{*}, & \partial_{t} p_{H_{z}, \ell}^{*}=j_{H_{z}, \ell}^{*}, \quad \ell=0, \ldots, n_{\psi} .
\end{array}
$$

Remark 4.11. There is no unique way to write the time-domain formulation depending on the choice of the family of auxiliary unknowns. However, all these choices are equivalent in terms of stability, see e.g. [55].

\section{Necessary and Sufficient Stability Conditions of PMLs for Models with LORENTZ PARAMETERS}

While Theorem 3.11 provides an explicit way to construct stable perfectly matched layers for anisotropic systems of type (18) by choosing $\psi(s)=a(s)$, such a choice of the function $\psi(s)$ may appear to be non-optimal, in a sense that it does not necessarily lead to the smallest number of unknowns in the resulting system in the time domain. On the other hand, while Lemma 3.6 provides a sufficient condition which should be satisfied by $\psi(s)$, it does not provide a constructive way of choosing such a function $\psi(s)$.

In the work [9], which is based on examining the behaviour of modes of the dispersion relation, a necessary condition of the stability of perfectly matched layers is formulated. Such a condition is easier to analyze compared to the conditions of Lemma 3.6, since instead of dealing with the right-half complex plane (as in Lemma 3.6), one studies the behaviour of functions restricted to the imaginary axis. It is well-known [10] that such conditions are not necessarily sufficient for the stability. For example, when applied to non-dispersive cases, they account only for the behaviour in the high-frequency regimes. Nevertheless, in [9] the authors have demonstrated that for a class of isotropic dispersive models (described by the sesquilinear form (18) with $a(s)=b(s))$ the necessary condition becomes sufficient. 
The goal of this section is to demonstrate that the necessary condition derived in [9] is sufficient for the stability of the perfectly matched layers for a more general class of models of the form (18), which, unlike the models considered in [9], are anisotropic. However, we restrict our considerations to passive materials only.

The results of this section will enable us to find a family of $\psi(s)$ that would result in stable PMLs, among which there is an optimal choice in terms of number of auxiliary unknowns in the resulting time-domain PML system. Finding such an optimal $\psi(s)$ is however not always trivial.

First of all, we recall the necessary stability condition derived in [9], and then formulate the main result (Theorem 5.12) in Section 5.2. The proof of this result can be found in Section 5.3. An easy to use method to construct the function $\psi(s)$ can be found in Appendix G. Finally, in Section 5.4 we show some numerical experiments with the new PML confirming the main results of Theorem 5.12.

\subsection{The Necessary Stability Condition}

A necessary stability condition of the perfectly matched layers for anisotropic dispersive medium had been deduced in [9] with the help of the plane-wave analysis. We do not repeat the derivation of this result for the system recast in the Laplace domain, but simply recall the result itself.

In this section we limit ourselves to passive (18) whose coefficients satisfy an extra condition. This is summarized in the following assumption, see also (14).

Assumption 5.1. The coefficients of the sesquilinear form (18) $a(s)^{-1}, b(s)^{-1}, c(s)$ are passive in the sense of Definition 2.1 and satisfy Assumption 4.1.

Remark 5.2. Since $a(s)^{-1}, b(s)^{-1}, c(s)$ satisfy Assumption 5.1, we know, thanks to Theorem 4.4, that they have a partial fraction expansion (50) and therefore correspond to Lorentz materials. However, the results of this section can be extended to the case when $a(s)^{-1}, b(s)^{-1}, c(s)$ are even meromorphic functions and have the partial fraction expansion as in (50) with infinite number of terms $(n=\infty)$, cf. e.g. [28, Theorem 1, $p$. 308]. Moreover, most of the results of this section do not rely on the (physical) assumption $a(s)=\varepsilon_{1}(s)^{-1}$, $b(s)=\varepsilon_{2}(s)^{-1}, c(s)=\mu(s) \rightarrow 1$ as $s \rightarrow \infty$. A numerical illustration of these two statements will be provided in Example 5.25.

Remark 5.3. If a passive function does not have poles and zeros s, s.t. $\operatorname{Re} s<0$ (i.e. non-dissipative case, cf. Definition 5.4), it is necessarily meromorphic, see e.g. [56].

Let us first recall a few definitions and crucial assumptions on the Maxwell system in the Laplace domain (5). First of all, the dispersion relation for this system is obtained when looking at the plane-wave solutions of (5). In particular, for Lorentz materials (60), it reads, see Appendix E:

$$
F(\omega, \mathbf{k})=\left(\omega^{-1} \tilde{a}(\omega) k_{x}^{2}+\omega^{-1} \tilde{b}(\omega) k_{y}^{2}-\omega \tilde{c}(\omega)\right) \prod_{\ell=0}^{n_{a}}\left(\omega^{2}-\omega_{a \ell}^{2}\right) \prod_{\ell=0}^{n_{b}}\left(\omega^{2}-\omega_{b \ell}^{2}\right) \prod_{\ell=0}^{n_{c}}\left(\omega^{2}-\omega_{c \ell}^{2}\right) \omega^{2}=0 .
$$

Here $\left(\omega_{a \ell}\right)_{\ell=0}^{n_{a}},\left(\omega_{b \ell}\right)_{\ell=0}^{n_{b}},\left(\omega_{c \ell}\right)_{\ell=0}^{n_{c}}$ are the poles of correspondingly $\tilde{a}, \tilde{b}$ and $\tilde{c}$.

Crucially, the analysis in [9] applies only to non-dissipative systems, as defined below.

Definition 5.4 ( $[9])$. A system is called non-dissipative if all modes $\omega(\mathbf{k})$ of its dispersion relation are real for all $\mathbf{k}=\left(k_{x}, k_{y}\right) \in \mathbb{R}^{2}$.

Proposition 5.5 (Non-dissipativity of (60a-60e)). Under Assumption 5.1, all modes $\omega(\mathbf{k})$ of (64) are real.

Proof. The solutions of the dispersion relation (64) are either poles of $\tilde{a}, \tilde{b}, \tilde{c}$, vanish, or are the solutions of

$$
\tilde{a}(\omega) k_{x}^{2}+\tilde{b}(\omega) k_{y}^{2}-\omega^{2} \tilde{c}(\omega)=0 .
$$

Thanks to Lemma 4.3 and passivity of $a^{-1}, b^{-1}, c$, the poles of $\tilde{a}, \tilde{b}, \tilde{c}$ are real.

It remains to show that $\omega(\mathbf{k})$ solving (65) are real. According to Proposition 2.18, all solutions of (65) satisfy $\operatorname{Im} \omega(\mathbf{k}) \leq 0$. Additionally, since $a, b, c$ are even, if $\omega$ solves $(65)$, so does $-\omega$. Therefore, $\omega(\mathbf{k}) \in \mathbb{R}$. 
The PML change of variables (39) leads to the system (62-63) with $\sigma(x)=\sigma=$ const, with a new dispersion relation, see Appendix $\mathrm{F}$ (and notice that $\varepsilon_{y}=a^{-1}$ and $\varepsilon_{x}=b^{-1}$ ),

$$
\begin{aligned}
F_{\sigma}(\sigma, \omega, \mathbf{k}) & =\left(\omega^{-1} \tilde{a}(\omega)\left(1-\frac{\sigma \tilde{\psi}(\omega)}{i \omega}\right)^{-2} k_{x}^{2}+\omega^{-1} \tilde{b}(\omega) k_{y}^{2}-\omega \tilde{c}(\omega)\right) \times \prod_{\ell}\left(\omega^{2}-\omega_{c \ell}^{2}\right) \\
& \times \prod_{\ell}\left(\omega^{2}-\omega_{\psi \ell}^{2}\right)^{2} \prod_{\ell}\left(\omega^{2}-\omega_{a \ell}^{2}\right) \prod_{\ell}\left(\omega^{2}-\omega_{b \ell}^{2}\right)\left(1+\sigma \tilde{\psi}(\omega)(-i \omega)^{-1}\right)^{2} \omega^{4}=0 .
\end{aligned}
$$

Here $\left(\omega_{\psi \ell}\right)_{\ell=0}^{n_{\psi}}$ are the poles of $\tilde{\psi}$. As in Section 2.3, one studies the stability of the PML system by examining the modes of the corresponding dispersion relation.

Definition 5.6 (Definition 4.3.2 in [9]). A PML system (62-63) obtained with the change of variables (39) is called uniformly stable if all solutions $\omega(\mathbf{k}, \sigma)$ of its dispersion relation (66) satisfy: $\operatorname{Im} \omega(\mathbf{k}, \sigma) \leq 0$, for all $\mathbf{k} \in \mathbb{R}^{2}$ and $\sigma \geq 0$.

To formulate the necessary PML stability condition, let us recall the concepts of phase and group velocities.

Definition 5.7. A phase velocity of a mode $\omega(\mathbf{k})$ is defined by $\mathbf{v}_{p}(\omega(\mathbf{k}))=\frac{\omega(\mathbf{k})}{|\mathbf{k}|} \frac{\mathbf{k}}{|\mathbf{k}|}$, and the group velocity by $\mathbf{v}_{g}(\omega(\mathbf{k}))=\nabla_{\mathbf{k}} \omega(\mathbf{k})$, provided that this derivative is well-defined.

Finally, the choice of the function $\psi$ in [9] is restricted to the class of functions $\tilde{\psi}(\omega)=h(\omega)$, where

$$
h(\omega)=1+\sum_{\ell=0}^{n} \frac{h_{\ell}}{\omega^{2}-\omega_{\ell}^{2}}, \quad h_{\ell} \in \mathbb{R}, \omega_{\ell} \in \mathbb{R} \backslash\{0\}, \quad \ell=0, \ldots, n .
$$

In [9] the authors have classified the solutions of the dispersion relation (66) and provided the necessary conditions of the uniform stability for each of the classes of the modes. We will discuss these classes afterwards.

Theorem 5.8 (Propositions 4.7, 4.8 in [9]; necessary PML stability condition). Given $\tilde{\psi}(\omega)=1+\sum_{\ell=0}^{n_{\psi}} \frac{\psi_{\ell}}{\omega^{2}-\omega_{\psi \ell}^{2}}$, with $\omega_{\psi \ell} \in \mathbb{R} \backslash\{0\}, \psi_{\ell} \in \mathbb{R}, \ell=0, \ldots, n_{\psi}$, let a PML system corresponding to the dispersion relation (66) be uniformly stable. Then

(N1) the coefficients of the expansion of $\tilde{\psi}(\omega)$ satisfy $\psi_{\ell}>0, \ell=0, \ldots, n_{\psi}$. Additionally, $\tilde{\psi}(0) \geq 0$.

(N2) for all the solutions $\omega_{j}(\mathbf{k}), j=1, \ldots, N$, of the original dispersion relation (64), it holds that

$$
\tilde{\psi}\left(\omega_{j}(\mathbf{k})\right) \mathbf{v}_{g, x}\left(\omega_{j}(\mathbf{k})\right) \mathbf{v}_{p, x}\left(\omega_{j}(\mathbf{k})\right) \geq 0, \mathbf{k} \in \mathbb{R}^{2} .
$$

Here the subscript $x$ indexes the $x$-component of a vector.

Let us re-interpret Theorem 5.8. All the modes of (66) belong to one of the following classes:

(1) they are non-propagating (i.e. independent of $\mathbf{k}$ ). Such modes can be real $\omega(\mathbf{k})=$ const $\in \mathbb{R}$ or solve

$$
1-(i \omega)^{-1} \tilde{\psi}(\omega) \sigma=0 .
$$

The stability of the modes that solve the above equation is ensured by the condition (N1), or, in our terms, cf. Theorem 4.5 , by the condition that $\operatorname{Re}(\bar{s} \psi(s))>0, s \in \mathbb{C}_{+}$.

(2) they are propagating, i.e. solve, for some $k_{x}, k_{y} \in \mathbb{R}$ :

$$
\omega^{-1} \tilde{a}(\omega)\left(1-\frac{\sigma \tilde{\psi}(\omega)}{i \omega}\right)^{-2} k_{x}^{2}+\omega^{-1} \tilde{b}(\omega) k_{y}^{2}-\omega \tilde{c}(\omega)=0 .
$$

A necessary condition of the stability of the propagating modes is given by the condition (N2). 
Let us rewrite (N2) with the use of the implicit function theorem. Clearly, this condition is of interest for the modes that depend on $\mathbf{k}$ non-trivially, i.e. modes that solve, cf. dispersion relation (64):

$$
\mathcal{F}(\omega, \mathbf{k})=\omega^{-1} \tilde{a}(\omega) k_{x}^{2}+\omega^{-1} \tilde{b}(\omega) k_{y}^{2}-\omega \tilde{c}(\omega)=0 .
$$

We denote the set of the propagating modes of the original system by

$$
\Omega_{p}:=\left\{\omega \in \mathbb{R}: \mathcal{F}(\omega, \mathbf{k})=0 \text { for some } \mathbf{k} \in \mathbb{R}^{2}\right\} .
$$

Then, given $\mathcal{F}(\omega, \mathbf{k})$ as in (69), we deduce with the help of the implicit function theorem:

$$
\partial_{k_{x}} \omega\left(k_{x}, k_{y}\right)=-\left(\partial_{k_{x}} \mathcal{F}(\omega, \mathbf{k})\right)\left(\partial_{\omega} \mathcal{F}(\omega, \mathbf{k})\right)^{-1}=-2 k_{x} \omega^{-1} \tilde{a}(\omega)\left(k_{x}^{2}\left(\omega^{-1} \tilde{a}(\omega)\right)^{\prime}+\left(\omega^{-1} \tilde{b}(\omega)\right)^{\prime} k_{y}^{2}-(\omega \tilde{c}(\omega))^{\prime}\right)^{-1} .
$$

The condition (N2) in Theorem 5.8 can be rewritten in the form: for all $\omega \in \Omega_{p}$,

$$
-k_{x}^{2} \tilde{\psi}(\omega(\mathbf{k})) \tilde{a}(\omega(\mathbf{k}))\left(k_{x}^{2}\left(\omega(\mathbf{k})^{-1} \tilde{a}(\omega(\mathbf{k}))\right)^{\prime}+k_{y}^{2}\left(\omega(\mathbf{k})^{-1} \tilde{b}(\omega(\mathbf{k}))\right)^{\prime}-(\omega(\mathbf{k}) \tilde{c}(\omega(\mathbf{k})))^{\prime}\right)^{-1} \geq 0
$$

provided that the above expression is well-defined.

We will need to reformulate the above condition in a simpler form. First of all, we will simplify (70). Given a rational function $r(z)$ satisfying Assumption 4.1, let us introduce a set $\mathcal{D}_{r}=\left\{\omega \in \mathbb{R}: \tilde{r}(\omega)=0\right.$ or $\left.\tilde{r}(\omega)^{-1}=0\right\}$.

Lemma 5.9. Let $a(s), b(s), c(s)$ satisfy Assumption 4.1. Then $\Omega_{p}$ defined in (70) can be represented as a union of the following (possibly intersecting) sets:

$$
\begin{aligned}
\Omega_{p} & =\Omega_{p}^{\tilde{a} \tilde{c}>0} \cup \Omega_{p}^{\tilde{a} \tilde{b}<0} \cup \mathcal{Z}_{p}, \\
\Omega_{p}^{\tilde{a} \tilde{c}>0} & =\left\{\omega \in \mathbb{R} \backslash\left(\mathcal{D}_{a} \cup \mathcal{D}_{c}\right): \tilde{a}(\omega) \tilde{c}(\omega)>0\right\}, \\
\Omega_{p}^{\tilde{a} \tilde{b}<0} & =\left\{\omega \in \mathbb{R} \backslash\left(\mathcal{D}_{a} \cup \mathcal{D}_{b}\right): \tilde{a}(\omega) \tilde{b}(\omega)<0\right\}, \\
\mathcal{Z}_{p} & =\left\{\omega \in \mathcal{D}_{a} \cup \mathcal{D}_{b} \cup \mathcal{D}_{c}: \text { for some } k_{x}, k_{y} \in \mathbb{R}, \text { it holds } \omega^{-1} \tilde{a}(\omega) k_{x}^{2}+\omega^{-1} \tilde{b}(\omega) k_{y}^{2}-\omega \tilde{c}(\omega)=0\right\} .
\end{aligned}
$$

Proof. First we show $\Omega_{p} \subset \Omega_{p}^{\tilde{a} \tilde{c}>0} \cup \Omega_{p}^{\tilde{a} \tilde{b}<0} \cup \mathcal{Z}_{p}$. Let $\omega \notin \mathcal{Z}_{p}$. Then the modes $\omega \in \Omega_{p}$ satisfy

$$
k_{x}^{2}=-\frac{\tilde{b}}{\tilde{a}} k_{y}^{2}+\omega^{2} \frac{\tilde{c}}{\tilde{a}}
$$

This equation has at least one real solution $k_{x}$ if and only if there exists $k_{y} \in \mathbb{R}$ s.t.

$$
-\tilde{b}(\omega) \tilde{a}(\omega)^{-1} k_{y}^{2}+\omega^{2} \tilde{c}(\omega) \tilde{a}(\omega)^{-1} \geq 0 .
$$

The above inequality has no real solutions $k_{y}$ if $\tilde{b}(\omega) \tilde{a}(\omega)^{-1} \geq 0$ and $\tilde{c}(\omega) \tilde{a}(\omega)^{-1}<0$. Hence, the inequality $(74)$ has a real solution $k_{y}$ if and only if $\omega \notin \mathcal{Z}_{p}$ satisfies at least one of the following conditions:

- $\tilde{b}(\omega) \tilde{a}(\omega)^{-1}<0$, i.e. $\omega \in \Omega_{p}^{\tilde{a} \tilde{b}<0}$

- $\tilde{c}(\omega) \tilde{a}(\omega)^{-1}>0$, i.e. $\omega \in \Omega_{p}^{\tilde{a} \tilde{c}>0}$.

The inclusion $\Omega_{p}^{\tilde{a} \tilde{c}>0} \cup \Omega_{p}^{\tilde{a} \tilde{b}<0} \cup \mathcal{Z}_{p} \subset \Omega_{p}$ can be proved similarly.

With the above we can reformulate the necessary stability condition (N2). 
Lemma 5.10 (Necessary stability condition (N2)). Let $a(s), b(s), c(s)$ satisfy Assumption 5.1, and let $\psi(s)$ satisfy Assumption 4.1 and $\operatorname{Re}(\bar{s} \psi(s))>0, s \in \mathbb{C}_{+}$. Let for $\omega \in \Omega_{p}$ the stability condition (71) hold true, provided that the latter expression is well-defined. Then for all $\omega \in \Omega_{p}^{\tilde{a} \tilde{c}>0} \cup \Omega_{p}^{\tilde{a} \tilde{b}<0}$ it holds

$$
\tilde{\psi}(\omega) \tilde{a}(\omega) \geq 0 .
$$

Proof. Consider the denominator of the left-hand side of $(71): \mathcal{H}:=k_{x}^{2}\left(\omega^{-1} \tilde{a}(\omega)\right)^{\prime}+k_{y}^{2}\left(\omega^{-1} \tilde{b}(\omega)\right)^{\prime}-(\omega \tilde{c}(\omega))^{\prime}$. Thanks to Lemmas 4.8, 4.9 about the signs of the derivatives of passive functions, for all $\omega \notin \mathcal{Z}_{p}$, it holds

$$
\left(\omega^{-1} \tilde{a}(\omega)\right)^{\prime}<0, \quad\left(\omega^{-1} \tilde{b}(\omega)\right)^{\prime}<0, \quad(\omega \tilde{c}(\omega))^{\prime}>0,
$$

Therefore, the denominator $\mathcal{H}<0$ for all $k_{x}, k_{y} \in \mathbb{R}$ and $\omega \notin \mathcal{Z}_{p}$. We can thus rewrite the inequality (71) as

$$
\tilde{\psi}(\omega) \tilde{a}(\omega) \geq 0 .
$$

\subsection{Equivalence of the Necessary and Sufficient Stability Conditions}

In this section we show that the necessary stability condition of the PML in Lemma 5.10 is equivalent to the sufficient stability condition of Lemma 3.6. To formulate it, we need the following assumption.

Assumption 5.11. The functions $a(s), b(s), c(s)$ satisfy Assumption 5.1, and $\psi(s)$ satisfies Assumption 4.1 and $\operatorname{Re}(\bar{s} \psi(s))>0, s \in \mathbb{C}_{+}$.

Theorem 5.12 (Equivalence of the necessary and sufficient stability conditions). Let $a(s), b(s), c(s), \psi(s)$ satisfy Assumption 5.11 and let

$$
A(u, v)=a(s)\left(\partial_{x} u, \partial_{x} v\right)+b(s)\left(\partial_{y} u, \partial_{y} v\right)+s^{2} c(s)(u, v)
$$

be the corresponding passive sesquilinear form. Then the following two conditions are equivalent:

(NSC1) for all $\omega \in \Omega_{p}^{\tilde{a} \tilde{b}<0} \cup \Omega_{p}^{\tilde{a} \tilde{c}>0}$ (see (72, 73)), it holds that $\tilde{\psi}(\omega) \tilde{a}(\omega) \geq 0$.

(NSC2) for all $\sigma \geq 0$, the sesquilinear form

$$
\tilde{A}_{\sigma}(u, v)=\frac{\psi(s)}{a(s)}\left(a(s)\left(1+\frac{\sigma \psi(s)}{s}\right)^{-2}\left(\partial_{x} u, \partial_{x} v\right)+b(s)\left(\partial_{y} u, \partial_{y} v\right)+s^{2} c(s)(u, v)\right), s \in \mathbb{C}_{+},
$$

is passive in the sense of Definition 2.11, and thus, bounded and coercive (due to Proposition 2.12).

Proof. The proof (NSC2) $\Longrightarrow$ (NSC1) follows from the results of [9]. Consider one of the factors of the dispersion relation (66) given by (68). Thanks to Proposition 2.18, the passivity of the sesqulinear form $\tilde{A}_{\sigma}(u, v)$ implies that all the solutions $\omega(\mathbf{k}), \mathbf{k} \in \mathbb{R}^{2}$, of (68) have a non-positive imaginary part, i.e. $\operatorname{Im} \omega(\mathbf{k}) \leq 0$. $\operatorname{In}[9$, Proposition 4.8], the following implication was shown:

$$
\text { all solutions } \omega(\mathbf{k}), \mathbf{k} \in \mathbb{R}^{2} \text { of }(68) \text { are s.t. } \operatorname{Im} \omega(\mathbf{k}) \leq 0 \Longrightarrow \text { for } \omega \in \Omega_{p},(71) \text { holds true. }
$$

Thanks to Lemma 5.10, from the above (NSC1) follows.

To show the implication (NSC1) $\Longrightarrow$ (NSC2), we use Lemma 3.6. It is sufficient to prove that for $\psi(s)$ satisfying the sign conditions (NSC1), it holds

$$
\begin{aligned}
& \operatorname{Re}\left(s c(s) a(s)^{-1} \psi(s)\right)>0, s \in \mathbb{C}_{+}, \\
& \operatorname{Re}\left(\bar{s} b(s) a(s)^{-1} \psi(s)\right)>0, s \in \mathbb{C}_{+} .
\end{aligned}
$$


The proof of the above relies on several technical results, which we will formulate and prove in Section 5.3.

We readily obtain the following corollary for a specific class of anisotropic systems (18) with $c(s)=b(s)^{-1}$. A particular representative of this class is a uniaxial cold plasma model given in (8), with $b(s)=1$.

Corollary 5.13. Let $a(s), \psi(s), b(s)$ satisfy Assumption 5.11. Then the following two conditions are equivalent:

(1) for all $\sigma \geq 0$, the sesquilinear form

$$
\tilde{A}_{\sigma}(u, v)=\frac{\psi(s)}{a(s)}\left(a(s)\left(1+\frac{\sigma \psi(s)}{s}\right)^{-2}\left(\partial_{x} u, \partial_{x} v\right)+b(s)\left(\partial_{y} u, \partial_{y} v\right)+s^{2} b(s)^{-1}(u, v)\right), s \in \mathbb{C}_{+},
$$

is passive in the sense of Definition 2.11.

(2) $\psi(s)=a(s)$.

Proof. Notice that the above sesquilinear form is as in Theorem 5.12 with $\tilde{c}(\omega)=\tilde{b}(\omega)^{-1}$. Using Theorem 5.12 , we see that in points $\omega \in \mathbb{R}$ s.t. $\tilde{a}(\omega) \tilde{c}(\omega)=\tilde{a}(\omega) \tilde{b}(\omega)^{-1}>0$, the functions $\tilde{\psi}(\omega)$ and $\tilde{a}(\omega)$ should be of the same sign. The same should hold in points where $\tilde{a}(\omega) \tilde{b}(\omega)<0$. Thus, the poles and zeros of $\psi(s)$ and $a(s)$ coincide (not necessarily with their multiplicity). Since all the poles and zeros of $\psi(s)$ are simple (with a possible exception of $s=0)$, see also $(\bar{M} 2)$ in Lemma 4.9, the assertion follows immediately.

Remark 5.14. From Theorem 3.11, there always exists $\tilde{\psi}$ which satisfies (NSC1) of Theorem 5.12, namely $\psi(s)=a(s)$, and thus, results in a stable PML.

\subsection{Proof of Theorem $\mathbf{5 . 1 2}$}

To prove Theorem 5.12, we proceed as follows. First of all, let us remark that the condition (NSC1) of Theorem 5.12 is equivalent to the following two conditions:

$$
\begin{aligned}
& \text { For all } \omega \in \Omega_{p}^{\tilde{a} \tilde{c}>0}: \tilde{\psi}(\omega) \tilde{a}(\omega) \geq 0, \\
& \text { For all } \omega \in \Omega_{p}^{\tilde{a} \tilde{b}<0}: \tilde{\psi}(\omega) \tilde{a}(\omega) \geq 0 .
\end{aligned}
$$

We will first demonstrate that (NSC1)-(a) implies the passivity condition (NSC2)-(a). To show that from (NSC1)-(b) the condition (NSC2)-(b) follows, we will make use of a logical argument (Lemma 5.21).

We proceed as follows:

(1) rewrite the condition (NSC2)-(a) in terms of equivalent conditions on the signs of $\tilde{\psi}$ in poles (zeros) of functions $\tilde{a}, \tilde{c}$ (Lemma 5.15);

(2) show that if $\tilde{\psi}$ satisfies (NSC1)-(a), then above conditions on the signs of $\tilde{\psi}$ follows. This is explained in Lemma 5.16, whose proof relies largely on Lemmas 5.18, 5.20 and Corollary 5.19.

\subsubsection{Reformulation of the Condition (NSC2)-(a)}

We start with the following simple lemma that allows us to reformulate the passivity condition (NSC2)-(a) in a more suitable form.

Lemma 5.15. Let $a(s), c(s), \psi(s)$ satisfy Assumption 5.11. Then (NSC2)-(a) holds if and only if the following limits exist and satisfy:

(a) in all poles $\left(\omega_{c_{j}}\right)_{j=0}^{n_{c}}$ of $\tilde{c}(\omega)$, it holds that $\lim _{\omega \rightarrow \omega_{c_{j}}} \tilde{a}(\omega)^{-1} \tilde{\psi}(\omega) \geq 0$.

(b) in all zeros $\left(\omega_{a_{j}}\right)_{j=0}^{n_{a}}$ of $\tilde{a}(\omega)$, it holds that $\lim _{\omega \rightarrow \omega_{a_{j}}} \tilde{\psi}(\omega) \tilde{c}(\omega) \geq 0$.

(c) in all poles $\left(\omega_{\psi_{j}}\right)_{j=0}^{n_{\psi}}$ of $\tilde{\psi}(\omega)$, it holds that $\lim _{\omega \rightarrow \omega_{\psi_{j}}} \tilde{a}(\omega)^{-1} \tilde{c}(\omega) \leq 0$. 
Proof. Thanks to Theorem 4.4, the function $c(s) a(s)^{-1} \psi(s)$ is passive if and only if it has the following partial fraction expansion:

$$
\tilde{c}(\omega) \tilde{a}(\omega)^{-1} \tilde{\psi}(\omega)=1-\sum_{\ell=0}^{N} \frac{p_{\ell}}{\omega^{2}-\omega_{\ell}^{2}}, p_{\ell}>0, \omega_{\ell} \in \mathbb{R}, \ell=0, \ldots N .
$$

Hence to show the equivalence of the statements, it is sufficient to ensure that in each of the poles $\omega=\omega_{k}, k=$ $0, \ldots, N$, the following limit exists and satisfies

$$
\lim _{\omega \rightarrow \omega_{k}}\left(\omega^{2}-\omega_{k}^{2}\right) \tilde{c}(\omega) \tilde{a}(\omega)^{-1} \tilde{\psi}(\omega)=-p_{k}<0 .
$$

Since $\left(\omega_{k}\right)_{k=0}^{N}$ is a subset of poles of $\tilde{c}, \tilde{a}^{-1}$ and $\tilde{\psi}$, we separately consider (76) for each of these poles. Let $\omega_{c_{j}}$ be a pole of $\tilde{c}(\omega)$. The latter function, thanks to Theorem 4.4, has an expansion $\tilde{c}(\omega)=1-\sum_{\ell=0}^{n_{c}} \frac{c_{\ell}}{\omega^{2}-\omega_{c_{\ell}}^{2}}$, where $c_{\ell}>0$ for $\ell=0 \ldots n_{c}$. Evaluating the limit $(76)$ in $\omega_{k}=\omega_{c_{j}}$, we obtain:

$$
\lim _{\omega \rightarrow \omega_{c_{j}}}\left(\omega^{2}-\omega_{c_{j}}^{2}\right) \tilde{c}(\omega) \tilde{a}(\omega)^{-1} \tilde{\psi}(\omega)=\lim _{\omega \rightarrow \omega_{c_{j}}}\left(\left(\omega^{2}-\omega_{c_{j}}^{2}\right) \tilde{c}(\omega)\right) \lim _{\omega \rightarrow \omega_{c_{j}}} \tilde{a}(\omega)^{-1} \tilde{\psi}(\omega)=-c_{j} \lim _{\omega \rightarrow \omega_{c_{j}}} \tilde{a}(\omega)^{-1} \tilde{\psi}(\omega) \leq 0 .
$$

The equality sign would mean that $c(s) a(s)^{-1} \psi(s)$ has no pole in $\omega_{c_{j}}$. Similarly we deduce the rest of the inequalities in the statement of the lemma.

\subsubsection{Necessary Stability Condition (NSC1)-(a) Implies Passivity of $c(s) a(s)^{-1} \psi(s)$}

Let us first present the main result of this section, which shows how from the necessary stability condition (NSC1)-(a) we obtain the passivity (NSC2)-(a).

Lemma 5.16. Let $a(s), \psi(s), c(s)$ satisfy Assumption 5.11. Assume that (NSC1)-(a) holds. Then (NSC2)-(a) holds true as well.

Proof. Due to Lemma 5.15, it is sufficient to analyze step by step the condition on $\psi$ in various cases: poles of $c$, zeros of $a$, as well as examine the values of $a, c$ in poles of $\psi$. Let us thus look at the following cases according to Lemma 5.15:

- given a pole $\omega_{c_{j}}$ of $\tilde{c}(\omega)$, we must show that the following limit exists and satisfies: $\lim _{\omega \rightarrow \omega_{c_{j}}} \tilde{a}(\omega)^{-1} \tilde{\psi}(\omega) \geq$ 0 . This follows by a direction application of Lemma 5.18, see below, with $\alpha=a, \gamma=c$ and $\phi=\psi$.

- given a zero $\omega_{a_{j}}$ of $\tilde{a}(\omega)$, we must demonstrate $\lim _{\omega \rightarrow \omega_{a_{j}}} \tilde{c}(\omega) \tilde{\psi}(\omega) \geq 0$. This is shown in Corollary 5.19, see below.

- finally, given a pole of $\omega_{\psi_{j}}$ of the function $\tilde{\psi}$, we obtain from Lemma 5.20 , see below, the existence of the following non-positive $\operatorname{limit} \lim _{\omega \rightarrow \omega_{\psi_{j}}} \tilde{c}(\omega) \tilde{a}(\omega)^{-1} \leq 0$.

Remark 5.17. The necessary condition in the above lemma is also sufficient, see Appendix D.

Before formulating results mentioned in the proof of Lemma 5.16, let us summarize simple properties of passive functions and their reciprocals crucial for proving the result of Theorem 5.12, see also Figure 6 for illustration. More precisely, for a passive rational function $r(s)$ satisfying Assumption 4.1, it holds, thanks to Lemmas 4.10, 4.8 and Theorem 4.4:

$(P 1)$ if $\omega=0$ is a pole of $\tilde{r}$, then $\tilde{r}(\omega)<0$ in a sufficiently small vicinity of $\omega=0$. Otherwise $\tilde{r}(0)>0$.

$(P 2)$ in a pole $\omega>0$, the function $\tilde{r}$ changes its sign from positive to negative.

$(P 3)$ in a zero $\omega>0$, the function $\tilde{r}$ changes its sign from negative to positive. 


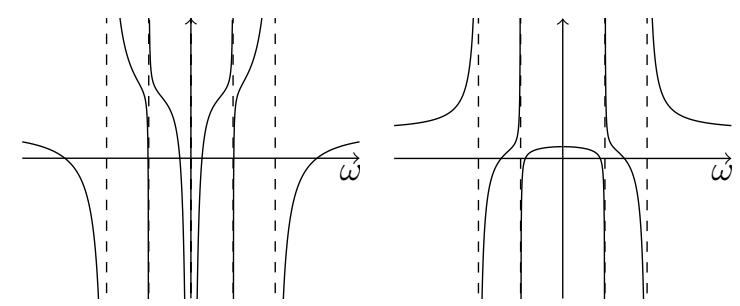

Figure 6 . In the left figure we depict $\tilde{r}(\omega)$, s.t. $\operatorname{Re}(s r(s))>0, s \in \mathbb{C}_{+}$and in the right one we plot $\tilde{r}(\omega)$, s.t. $r(s)$ satisfies $\operatorname{Re}(\bar{s} r(s))>0, s \in \mathbb{C}_{+}$. In both cases $r(s)$ is a rational function satisfying Assumption 4.1.

Similarly, for a rational function $r(s)$ satisfying Assumption 4.1, whose reciprocal is passive, i.e. $\operatorname{Re}(\bar{s} r(s))>0$, $s \in \mathbb{C}_{+}$, it holds, thanks to Lemmas 4.10, 4.9, Theorem 4.5:

$(\bar{P} 1)$ if $\omega=0$ is a zero of $\tilde{r}$, then $\tilde{r}(\omega)<0$ in a sufficiently small vicinity of $\omega=0$. Otherwise $\tilde{r}(0)>0$.

$(\bar{P} 2)$ in a pole $\omega>0$, the function $\tilde{r}$ changes its sign from negative to positive.

$(\bar{P} 3)$ in a zero $\omega>0$, the function $\tilde{r}$ changes its sign from positive to negative.

Now let us state the auxiliary results used in the proof of Lemma 5.16.

Lemma 5.18. Let $\alpha(s), \phi(s), \gamma(s)$ satisfy Assumption 4.1, and $\operatorname{Re}(\bar{s} \alpha(s))>0, \operatorname{Re}(\bar{s} \phi(s))>0$, and $\operatorname{Re}(s \gamma(s))>0$, for all $s \in \mathbb{C}_{+}$. Assume that for all $\omega \in \mathbb{R} \backslash\left(\mathcal{D}_{\gamma} \cup \mathcal{D}_{\alpha}\right)$ it holds

$$
\tilde{\gamma}(\omega) \tilde{\alpha}(\omega)>0 \Longrightarrow \tilde{\phi}(\omega) \tilde{\alpha}(\omega) \geq 0 .
$$

Then in all the poles $\left(\omega_{\gamma_{j}}\right)_{j=0}^{n_{\gamma}}$ of $\tilde{\gamma}(\omega)$ the following limit exists and satisfies

$$
0 \leq \lim _{\omega \rightarrow \omega_{\gamma_{j}}} \tilde{\alpha}(\omega)^{-1} \tilde{\phi}(\omega)<+\infty
$$

Proof. Let $\omega_{\gamma_{j}} \geq 0$ be a pole of $\tilde{\gamma}(\omega)$ (negative poles are treated similarly). Then the following cases are possible:

- $\omega_{\gamma_{j}}=0$. According to Theorem 4.5 applied to $\alpha(s)$, only one of the following can hold true:

(1) either $\tilde{\alpha}(0)=0$. Then, for sufficiently small $\omega>0$ :

$-\tilde{\alpha}(\omega)^{-1}<0$, see $(\bar{P} 1)$,

$-\tilde{\gamma}(\omega)<0$, see $(P 1)$.

Thus, in a small vicinity of zero, $\tilde{\alpha} \tilde{\gamma}>0$, and with $(77)$ it follows that there $\tilde{\phi}(\omega) \leq 0$, which is possible only if $\tilde{\phi}(0)=0$, see $(\bar{P} 1)$. Thus, $\tilde{\phi}$, $\tilde{\alpha}$ both have a zero of order 2 in $\omega=0$, see $(\bar{M} 2)$ of Lemma 4.9. Therefore, the following limit exists and satisfies: $\lim _{\omega \rightarrow 0} \tilde{\phi}(\omega) \tilde{\alpha}(\omega)^{-1}>0$.

(2) or $\tilde{\alpha}(0)^{-1}>0$. Applying Theorem 4.5 to $\phi(s)$, we notice $\tilde{\phi}(0) \geq 0$, hence (78) holds true.

- $\omega_{\gamma_{j}}>0$. The following cases are possible:

(1) $0<\tilde{\alpha}\left(\omega_{\gamma_{j}}\right)<\infty\left(-\infty<\tilde{\alpha}\left(\omega_{\gamma_{j}}\right)<0\right)$. Due to $(P 2)$, the inequality $\tilde{\gamma}(\omega) \tilde{\alpha}(\omega)>0$ holds for all $\omega=\omega_{\gamma_{j}}-\delta\left(\omega=\omega_{\gamma_{j}}+\delta\right)$, with $\delta>0$ being sufficiently small. As for $\tilde{\phi}$, there are two possibilities:

- $\tilde{\phi}(\omega)$ does not have a pole in $\omega_{\gamma_{j}}$. Then, due to (77), the limit (78) exists and satisfies the inequality (78) (this includes the case $\tilde{\phi}\left(\omega_{\gamma_{j}}\right)=0$ ).

- $\tilde{\phi}$ has a pole in $\omega_{\gamma_{j}}$. This is impossible, since in this case (77) would require that $\tilde{\phi}(\omega)>0$ $(\tilde{\phi}(\omega)<0)$ for $\omega=\omega_{\gamma_{j}}-\delta\left(\omega=\omega_{\gamma_{j}}+\delta\right)$, for $\delta>0$; this would be a contradiction to the fact that in its pole $\tilde{\phi}(\omega)$ changes its sign from negative to positive, see $(\bar{P} 2)$.

(2) $\tilde{\alpha}(\omega)$ has a zero in $\omega_{\gamma_{j}}$. Then in $\omega_{\gamma_{j}} \tilde{\alpha}(\omega)$ changes its sign from positive to negative, see $(\bar{P} 3)$, and so does $\tilde{\gamma}$, see $(P 2)$. Thus, in the vicinity of $\omega_{\gamma_{j}}$, the product $\tilde{\alpha}^{-1}(\omega) \tilde{\gamma}(\omega)>0$. Therefore, $(77)$ 
requires that $\tilde{\phi}(\omega) \geq 0$ there. Thus $\tilde{\phi}$ changes the sign in $\omega_{\gamma_{j}}$ from positive to negative, which is possible only if $\tilde{\phi}\left(\omega_{\gamma_{j}}\right)=0$, see $(\bar{P} 3)$. Since nonvanishing zeros of $\tilde{\alpha}, \tilde{\psi}$ are simple, (78) holds true. (3) $\tilde{\alpha}$ has a pole in $\omega_{\gamma_{j}}$. Then there are two possibilities:

$-\tilde{\phi}$ is finite in $\omega_{\gamma_{j}}$. This immediately implies that the limit (78) vanishes.

- $\tilde{\phi}$ has a pole in $\omega_{\gamma_{j}}$. Due to $(\bar{P} 2)$, in $\omega_{\gamma_{j}}$ both functions $\tilde{\phi}$, $\tilde{\alpha}$ change their signs from negative to positive (and hence their product is positive in the vicinity of the pole). This, combined with the simplicity of poles of $\tilde{\alpha}, \tilde{\phi}$, shows that (78) holds true.

The following result is a direct corollary of the above.

Corollary 5.19. Let $a(s), \psi(s), c(s)$ satisfy Assumption 5.11. Assume that (NSC1)-(a) holds true. Then in all the zeros $\left(\omega_{a_{j}}\right)_{j=0}^{n_{a}}$ of $\tilde{a}(\omega)$ the following limit exists and satisfies

$$
0 \leq \lim _{\omega \rightarrow \omega_{a_{j}}} \tilde{c}(\omega) \tilde{\psi}(\omega)<+\infty
$$

Proof. The condition (NSC1)-(a) can be rewritten as follows: for all $\omega \in \mathbb{R} \backslash\left(\mathcal{D}_{a} \cup \mathcal{D}_{c}\right)$, it holds: $\tilde{c}(\omega)^{-1} \tilde{a}(\omega)^{-1}>$ $0 \Longrightarrow \tilde{\psi}(\omega) \tilde{c}(\omega)^{-1} \geq 0$. Then $\alpha(s)=c(s)^{-1}, \gamma(s)=a(s)^{-1}$ and $\phi(s)=\psi(s)$ satisfy conditions of Lemma 5.18 (see (14)), from which the result is immediately obtained.

Finally, what remains to show is the following.

Lemma 5.20. Let $a(s), \psi(s), c(s)$ satisfy Assumption 5.11. Assume that (NSC1)-(a) holds. Then in all the poles $\left(\omega_{\psi_{j}}\right)_{j=0}^{n_{\psi}}$ of $\tilde{\psi}(\omega)$, the following limit exists and satisfies

$$
-\infty<\lim _{\omega \rightarrow \omega_{\psi_{j}}} \tilde{c}(\omega) \tilde{a}(\omega)^{-1} \leq 0
$$

Proof. Recall that $\omega_{\psi_{j}}=0$ cannot be a pole of $\tilde{\psi}$, see Theorem 4.5. Hence we consider the case $\omega_{\psi_{j}}>0$ (negative values are treated similarly). We thus look at the following cases:

(1) let $-\infty<\tilde{c}\left(\omega_{\psi_{j}}\right)<0\left(0<\tilde{c}\left(\omega_{\psi_{j}}\right)<\infty\right)$. As for $\tilde{a}(\omega)$, it satisfies either of the following:

- $\tilde{a}(\omega)$ is finite and does not change its sign in $\omega_{\psi_{j}}$. Assume by contradiction that (80) does not hold true, i.e. $\tilde{c}\left(\omega_{\psi_{j}}\right) \tilde{a}\left(\omega_{\psi_{j}}\right)^{-1}>0$. By continuity this holds in a vicinity of $\omega_{\psi_{j}}$. Then, due to (NSC1)-(a), $\tilde{a}(\omega) \tilde{\psi}(\omega) \geq 0$ in the vicinity of $\omega_{\psi_{j}}$. We arrive at the contradiction, since $\tilde{\psi}$ changes its sign in $\omega_{\psi_{j}}$ but not $\tilde{a}$. Hence, necessarily, $\tilde{c}\left(\omega_{\psi_{j}}\right) \tilde{a}\left(\omega_{\psi_{j}}\right)^{-1} \leq 0$.

- $\omega_{\psi_{j}}$ is a pole of $\tilde{a}(\omega)$. Then obviously the limit (80) vanishes.

- $\tilde{a}\left(\omega_{\psi_{j}}\right)=0$ : due to $(\bar{P} 3), \tilde{a}$ changes its sign from positive to negative in $\omega_{\psi_{j}}$, and the inequality $\tilde{c}(\omega) \tilde{a}(\omega)>0$ holds for all $\omega=\omega_{\gamma_{j}}+\delta\left(\omega=\omega_{\gamma_{j}}-\delta\right)$, with $\delta>0$ being sufficiently small. Then, due to (NSC1)-(a), the product $\tilde{a}(\omega) \tilde{\psi}(\omega) \geq 0$ for such values $\omega$, which is impossible, since $\tilde{\psi}$ changes in $\omega_{\psi_{j}}$ its sign from negative to positive and $\tilde{a}$ from positive to negative, see $(\bar{P} 3)$ and $(\bar{P} 2)$.

(2) $\tilde{c}\left(\omega_{\psi_{j}}\right)=0$. Then either is possible for $\tilde{a}$ :

- $\tilde{a}(\omega)$ is finite or has a pole in $\omega_{\psi_{j}}$. Obviously, the limit (80) vanishes.

- if $\tilde{a}\left(\omega_{\psi_{j}}\right)=0$, then $(P 3)$ and $(\bar{P} 2)$ imply that $\tilde{a}(\omega) \tilde{c}(\omega)<0$ in a vicinity of $\omega_{\psi_{j}}$. Combined with the fact that positive zeros and poles of $\tilde{a}$ and $\tilde{c}$ are simple, we obtain the existence of the limit.

(3) $\tilde{c}$ has a pole in $\omega_{\psi_{j}}$. As shown in Lemma 5.18, with $\alpha=a, \phi=\psi, \gamma=c$, in this case $\tilde{\psi}$ can have a pole in $\omega_{\psi_{j}}$ only if $\tilde{a}$ has a pole in $\omega_{\psi_{j}}$. The existence of the limit follows from the fact that $\omega_{\psi_{j}}$ is a simple pole of $\tilde{a}, \tilde{c}$, and the sign of the limit follows from the character of the sign change of $\tilde{c}$ and $\tilde{a}$ in $\omega_{\psi_{j}}$, see $(P 2)$ and $(\bar{P} 2)$. 
5.3.3. Necessary Stability Condition (NSC1)-(b) Implies Passivity of $b(s)^{-1} a(s) \psi(s)^{-1}$ (NSC2)-(b)

Now our goal is to connect the passivity of $b(s)^{-1} a(s) \psi(s)^{-1}$ and the necessary stability condition. We will not make use of lemmas similar to Lemma 5.18, but rather use some trivial logic arguments to show that Lemma 5.16 implies the validity of the following result.

Lemma 5.21. Let $a(s), \psi(s), b(s)$ satisfy Assumption 5.11. If (NSC1)-(b) holds true, then so does (NSC2)-(b). Proof. Given $a, b, \psi$, let us introduce the following new unknowns:

$$
c_{n}:=\psi^{-1}, a_{n}:=b, \psi_{n}:=a .
$$

Notice that $c_{n}(s)$ is passive, see (14). Due to Lemma 5.16, the first statement below implies the second one:

(1) for all $\omega \in \mathbb{R} \backslash\left(\mathcal{D}_{a_{n}} \cup \mathcal{D}_{c_{n}}\right)$ it holds that

$$
\tilde{c}_{n} \tilde{a}_{n}>0 \Longrightarrow \tilde{\psi}_{n} \tilde{a}_{n} \geq 0
$$

(2) $\operatorname{Re}\left(s c_{n}(s) a_{n}(s)^{-1} \psi_{n}(s)\right)>0$ in $\mathbb{C}_{+}$, or, equivalently, see (14), and the notation (81), $\operatorname{Re}\left(\bar{s} b(s) \psi(s) a(s)^{-1}\right)>$ 0 in $\mathbb{C}_{+}$.

With the new notation (81), the expression (82) reads

$$
\tilde{\psi}(\omega)^{-1} \tilde{b}(\omega)>0 \Longrightarrow \tilde{a}(\omega) \tilde{b}(\omega) \geq 0, \quad \omega \in \mathbb{R} \backslash\left(\mathcal{D}_{\psi} \cup \mathcal{D}_{b}\right),
$$

However, the above is equivalent to

$$
\tilde{a}(\omega) \tilde{b}(\omega)<0 \Longrightarrow \tilde{\psi}(\omega)^{-1} \tilde{b}(\omega)^{-1} \leq 0, \quad \omega \in \mathbb{R} \backslash\left(\mathcal{D}_{\psi} \cup \mathcal{D}_{b}\right),
$$

or, alternatively, due to the continuity of all the functions in $\omega$,

$$
\tilde{a}(\omega) \tilde{b}(\omega)<0 \Longrightarrow \tilde{\psi}(\omega) \tilde{a}(\omega) \leq 0, \omega \in \mathbb{R} \backslash\left(\mathcal{D}_{a} \cup \mathcal{D}_{b}\right)
$$

From this we obtain the desired statement.

Remark 5.22. As before, the necessary condition in the above lemma is also sufficient, see Remark 5.17.

\subsection{Numerical Experiments}

In this section we will numerically verify Theorem 5.12, and study its applicability to materials with losses.

Example 5.23. (Anisotropic Lorentz material. Numerical verification of Theorem 5.12) We start with the system (5), where we choose the parameters as follows, see Remark 2.6,

$$
\tilde{a}(\omega)=\tilde{\varepsilon}_{2}(\omega)^{-1}=\frac{\omega^{2}}{\omega^{2}-\omega_{p}^{2}}, \quad \tilde{b}(\omega)=\tilde{\varepsilon}_{1}(\omega)^{-1}=\frac{\omega^{2}-\frac{\omega_{p}^{2}}{4}}{\omega^{2}-4 \omega_{p}^{2}}, \quad \tilde{c}(\omega)=\tilde{\mu}(\omega)=1, \quad \omega_{p}=5 .
$$

By Theorem 5.12, stable PML in $x$ is generated by $\psi(s)$, s.t. $\psi(s)^{-1}$ is passive and $\tilde{\psi}(\omega) \tilde{a}(\omega) \geq 0$ for $\omega$ from

$$
\Omega_{p}^{\tilde{a} \tilde{b}<0} \cup \Omega^{\tilde{a} \tilde{c}>0}=\left(-\infty,-\omega_{p}\right) \cup\left(-\frac{\omega_{p}}{2}, 0\right) \cup\left(0, \frac{\omega_{p}}{2}\right) \cup\left(\omega_{p},+\infty\right) .
$$

Let us consider the following possible choices of $\psi(s)$ :

$$
\psi_{1}(s)=1, \quad \tilde{\psi}_{2}(\omega)=\frac{\omega^{2}}{\omega^{2}-\frac{\omega_{p}^{2}}{4}}, \quad \tilde{\psi}_{3}(\omega)=\frac{\omega^{2}\left(\omega^{2}-\frac{\omega_{p}^{2}}{4}\right)}{\left(\omega^{2}-\frac{\omega_{p}^{2}}{8}\right)\left(\omega^{2}-\omega_{p}^{2}\right)}, \quad \psi_{4}(s)=\varepsilon_{2}(s)^{-1} .
$$


Notice that $\operatorname{Re}\left(\bar{s} \psi_{j}(s)\right)>0, j=1, \ldots, 4$, in $\mathbb{C}_{+}$thanks to $(\bar{M} 2)$ of Lemma 4.9. We expect the PMLs to be unstable for $\psi(s)=\psi_{1}(s)$ (classical PMLs) and stable otherwise (since $\tilde{\psi}_{j} \tilde{a} \geq 0, j=2,3,4$, in $\Omega_{p}^{\tilde{a} \tilde{b}<0} \cup \Omega^{\tilde{a} \tilde{c}>0}$ ). In particular, $\psi_{4}$ corresponds to the PML of Theorem 3.11. The parameters of the numerical experiment are given in Table 5 . As before, in $y$-direction we use zero Dirichlet boundary conditions.

\begin{tabular}{ccccccccc}
$L_{x}$ & $L_{y}$ & $L_{x}^{\sigma}$ & $L_{y}^{\sigma}$ & $\sigma_{x}(x)$ & $\sigma_{y}(y)$ & $\Delta x$ & $\Delta t$ & $f(t, x, y)$ \\
\hline 8 & 12 & 2 & 0 & $20 x^{2}$ & 0 & 0.025 & 0.0125 & $(t-2) \mathrm{e}^{-10(t-2)^{2}-1000(x-3.8)^{2}-2 z^{2}} H(3.98-x)$
\end{tabular}

TABle 5. Parameters for the experiment with dielectric permittivity and magnetic permeability (83). By $H(x)$ we denote the Heaviside function. See Section 3.1.2 for notation.

The results of these experiments are shown in Figure 7.
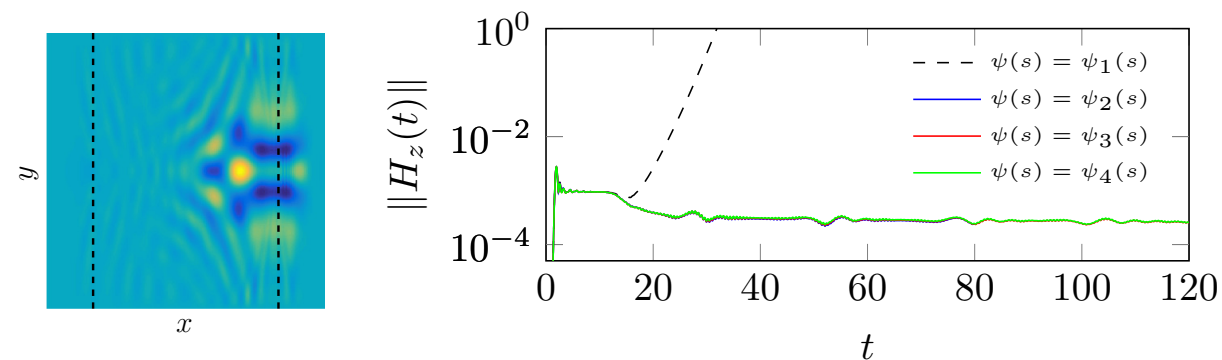

Figure 7. Left: $H_{z}$ of Example 5.23 at $t=60$ computed with $\psi(s)=\psi_{2}(s)$, see (84). The boundary between the physical domain and the PML is marked in black. Right: the dependence of $\left\|H_{z}\right\|_{L^{2}}$, computed with different $\psi(s)$, on time.

The new PMLs are stable, unlike the classical PMLs. The rate of the decrease of the solution norm for the PMLs with $\psi(s)=\psi_{2}(s), \psi(s)=\psi_{3}(s), \psi(s)=\psi_{4}(s)$ is almost indistinguishable on the scale used in the figure.

Example 5.24. (Anisotropic Lorentz Materials with Loss) Another interesting question is whether a perfectly matched layer which we have devised for the Lorentz materials (9) in this section can be applied to Lorentz materials with losses, more precisely, when the dielectric permittivity/permeability are of the form

$$
r(s)=1+\sum_{\ell=0}^{n} \frac{r_{\ell}}{s^{2}+2 \nu_{\ell} s+\omega_{\ell}^{2}}, \quad r_{\ell}>0, \omega_{\ell} \geq 0, \nu_{\ell} \geq 0 .
$$

It is easy to check that $\operatorname{Re}(s r(s))>0, s \in \mathbb{C}_{+}$; this shows that all the roots of $r(s)$ lie in $\mathbb{C} \backslash \mathbb{C}_{+}$. Moreover, all poles of $r(s)$ also lie in $\mathbb{C} \backslash \mathbb{C}_{+}$. To see this, notice that the poles of $r(s)$ are given by $s_{\ell}^{ \pm}=-\nu_{\ell} \pm \sqrt{\nu_{\ell}^{2}-\omega_{\ell}^{2}}, \ell=$ $0, \ldots, n$. If $\nu_{\ell} \geq \omega_{\ell}$, then $s_{\ell}^{ \pm} \leq 0$; otherwise $\operatorname{Re} s_{\ell}^{ \pm}=-\nu_{\ell}$.

We consider the problem (5) with the following parameters:

$$
\varepsilon_{1}(s)=1, \quad \varepsilon_{2}(s)=1+\frac{\varepsilon_{21}}{s^{2}+2 \nu s+\omega_{p}^{2}}, \quad \mu(s)=1, \quad \varepsilon_{21}=12, \quad \omega_{p}=2 .
$$

Although these parameters do not satisfy Assumption 4.1, we would like to verify whether it's possible to use for it the PML in $x$-direction, which was constructed for the analogical non-dissipative case, namely $\psi(s)=\varepsilon_{2}(s)^{-1}$ from (85) with $\nu=0$. The necessary stability conditions of the PMLs in [9] do not cover this case, and hence 
we would like to check it numerically. We test the following choices of $\psi(s)=\psi_{j}(s)$ :

$$
\psi_{1}(s)=1, \quad \psi_{2}(s)=\left(1+\frac{\varepsilon_{21}}{s^{2}+\omega_{p}^{2}}\right)^{-1}, \quad \psi_{3}(s)=\left(1+\frac{\varepsilon_{21}}{s^{2}+2 \nu s+\omega_{p}^{2}}\right)^{-1} .
$$

The choice $\psi(s)=\psi_{2}(s)$ corresponds to a stable PML in the case $\nu=0$, whereas the choice $\psi(s)=\psi_{3}(s)$ is a stable PML of Theorem 3.11. The parameters of the experiment are presented in Table 6.

\begin{tabular}{ccccccccc}
$L_{x}$ & $L_{y}$ & $L_{x}^{\sigma}$ & $L_{y}^{\sigma}$ & $\sigma_{x}(x)$ & $\sigma_{y}(y)$ & $\Delta x$ & $\Delta t$ & $f(t, x, y)$ \\
\hline 8 & 12 & 2 & 0 & $50 x^{2}$ & 0 & 0.05 & 0.025 & $\mathrm{e}^{-1000(x-3.8)^{2}-300 y^{2}-300 z^{2}} H(3.98-x) \mathrm{e}^{-15(t-1.6)^{2}}$
\end{tabular}

TABLE 6. Parameters for the experiment of Example 5.24 with the dielectric permittivity and magnetic permeability (85). By $H(x)$ we denote the Heaviside function. The notation can be found in the beginning of Section 3.1.2.
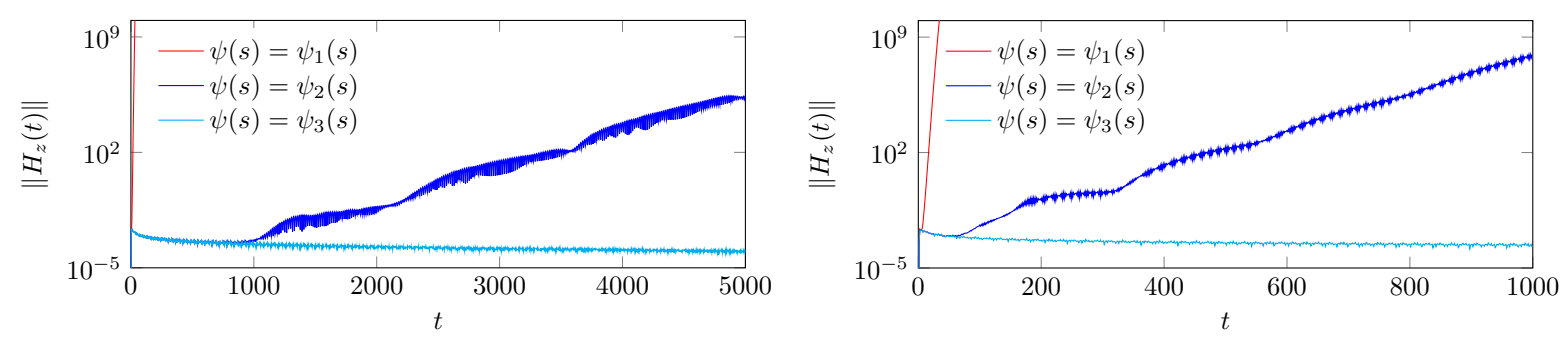

FiguRE 8. The dependence of the $L^{2}$-norm of the solution $H_{z}$ (computed inside the domain and the PML) to the problem of Example 5.24 on time, computed with different PMLs. In the left plot $\nu=0.01$ in (85), and in the right plot $\nu=0.1$.

The results of the experiment are shown in Figure 8. The choice $\psi(s)=\psi_{3}(s)$ results in a stable solution, thus confirming numerically the result of Theorem 3.11 (even though here $\sigma(x) \neq$ const). The choice $\psi(s)=\psi_{2}(s)$, which one would hope to be stable, results in instabilities, which seem to develop at long time. However, the larger the absorption $\nu$ is, the faster the instability occurs. The classical PMLs develop instabilities almost immediately.

Example 5.25. (Nonlocal Materials) One of the examples of a function that satisfies the passivity requirement $\operatorname{Re}(s f(s))>0, s \in \mathbb{C}_{+}$, is [57, p.19] a function $s^{-1} \tanh (s)$, which has the following partial fraction expansion [58, 1.421 .2$, p.43]:

$$
s^{-1} \tanh (s)=2 \sum_{\ell=1}^{\infty}\left(s^{2}+(2 \ell-1)^{2}\left(\frac{\pi}{2}\right)^{2}\right)^{-1}
$$

One can see that the above expression coincides, up to a constant, with the expansion (50) where $n=\infty$. We would like to apply the results of Theorem 5.12 to the system (5) with parameters, see also Remark 5.2:

$$
\varepsilon_{1}(s)=s^{-1} \tanh (4 s), \quad \varepsilon_{2}(s)=s^{-1} \tanh (2 s), \quad \mu(s)=s^{-1} \tanh (s) .
$$

Notice that these functions do not satisfy the behaviour at the infinity as prescribed by Assumption 4.1. Our goal is to demonstrate numerically that the statement of Theorem 5.12 can be extended to this case. More 
precisely, we would like to construct the PML in the direction $x$; in the direction $y$ we will use zero boundary conditions.

One can notice that on the interval $\omega \in\left(\frac{3 \pi}{8}, \frac{\pi}{2}\right)$, it holds

$$
\tilde{\varepsilon}_{1}(\omega)<0, \tilde{\varepsilon}_{2}(\omega)<0, \tilde{\mu}(\omega)>0 \text {. }
$$

According to Theorem 5.12, the sign of the function $\tilde{\psi}(\omega)$ on this interval can be arbitrary (in particular, positive, so that it does not coincide with the sign of $\tilde{\varepsilon}_{2}(\omega)$ ). We thus suggest that the choices of $\psi(s)=\psi_{2,3}(s)$ for the PML (32) in the direction $x$ should lead to a stable method, and the choice $\psi(s)=1$ would be unstable:

$$
\psi_{1}(s)=1, \quad \psi_{2}(s)=\varepsilon_{2}(s)^{-1}, \quad \psi_{3}(s)=\varepsilon_{2}(s)^{-1}\left(s^{2}+\left(\frac{3 \pi}{8}\right)^{2}\right)^{-1}\left(s^{2}+\left(\frac{\pi}{2}\right)^{2}\right) .
$$

One can check that $\tilde{\psi}_{3}(\omega)$ is positive on $\left(\frac{3 \pi}{8}, \frac{\pi}{2}\right)$, and its sign coincides on the rest of the real axis with $\tilde{\varepsilon}_{2}(\omega)$.

To verify the condition that $\operatorname{Re}\left(\bar{s} \psi_{3}(s)\right)>0$ in $\mathbb{C}_{+}$, we refer to an extension of Lemma 4.10 for a certain class of meromorphic functions, see [28, p.308, Theorem 1], as well as [59, pp.140-143] for a more detailed proof of the corresponding result.

To explain this in more detail, let us consider the aforementioned theorem, which is originally due to Krein.

Theorem 5.26 (Theorem of Krein, [28]). In order that a meromorphic function $\phi(z)$, s.t. $\phi(z)$ is real on $\mathbb{R}$, map the upper half-plane into the upper half-plane, it is necessary and sufficient that this function be represented in the form:

$$
\phi(z)=C \frac{z-a_{0}}{z-b_{0}} \prod_{k \in \mathbb{Z} \backslash\{0\}}\left(1-\frac{z}{a_{k}}\right)\left(1-\frac{z}{b_{k}}\right)^{-1},
$$

with $C>0, b_{k}<a_{k}<b_{k+1}$, for all $k \in \mathbb{Z}$ and $a_{-1}<0<b_{1}$.

It is easy to see that given any even passive meromorphic function $c(s)$, which is real on the imaginary axis, we can define $h_{c}(z)=z c(-i z)$, with $z \in \mathbb{C}$ which would satisfy the conditions of the above theorem. Moreover, $h_{c}(z)$ maps the upper half plane into the upper half plane if and only if $c(z)$ is passive. In particular, for $z$ s.t. $\operatorname{Im} z>0$, it holds

$$
\operatorname{Im} h_{c}(z)=\operatorname{Re}\left(-i h_{c}(z)\right)=\operatorname{Re}(-i z c(-i z))>0, \quad \operatorname{Re}(-i z)=\operatorname{Im} z>0 .
$$

Moreover, such $h_{c}(z)$ is real on the real axis. The application of the Krein theorem gives the following representation:

$$
\tilde{c}(z)=c(-i z)=C z^{-1} \frac{z-a_{0}}{z-b_{0}} \prod_{k \in \mathbb{Z} \backslash\{0\}}\left(1-\frac{z}{a_{k}}\right)\left(1-\frac{z}{b_{k}}\right)^{-1}
$$

If $\tilde{c}(z)$ is even, because of symmetry of the poles and roots of $\tilde{c}(z)$ with respect to zero, only two possibilities are possible:

(1) either $a_{0}=0$; from this $b_{k}=b_{-k+1}$, when $k \in \mathbb{Z}$ and $a_{k}=a_{-k}, k \in \mathbb{Z} \backslash\{0\}$;

(2) either $b_{0}=0$; from this $a_{k}=a_{-k-1}$, for $k \in \mathbb{Z}$, and $b_{k}=b_{-k}$ for $k \in \mathbb{Z} \backslash\{0\}$.

In all other cases it is impossible to place zeros and poles of $\tilde{c}(z)$ symmetrically with respect to zero.

In particular, for $\tilde{\varepsilon}_{2}(\omega)$, the root $a_{0}=0$, and the other roots $a_{k}=\frac{\pi k}{2}$, with $k \in \mathbb{Z} \backslash 0$. Similarly, the pole $b_{0}=-\frac{\pi}{4}$, and $b_{k}=\frac{\pi}{4}+\frac{\pi}{2}(k-1)=\frac{\pi}{4}(2 k-1)$, for $k \in \mathbb{Z} \backslash\{0\}$. 
Finding $C$ in $(91)$ from the condition $\varepsilon_{2}(0)=2$, we obtain the following expansion of $\varepsilon_{2}(s)$ :

$$
\varepsilon_{2}(s)=\frac{\pi}{2 i\left(s-i \frac{\pi}{4}\right)} \prod_{k \in \mathbb{Z} \backslash\{0\}}\left(1+\frac{2 s}{i \pi k}\right)\left(1+\frac{4 s}{i(2 k-1) \pi}\right)^{-1} .
$$

From this expansion for $\varepsilon_{2}(s)$ we can obtain a similar product expansion for $\psi_{3}(s)$ (thus showing that $h_{\psi_{3}}(z)$ is of the form (90). Notice that the poles and roots of $\tilde{\psi}_{3}(\omega)$ interlace. The non-negative roots of $\tilde{\psi}_{3}(\omega)$ are $\frac{\pi}{4}, \frac{3 \pi}{4}, \frac{5 \pi}{4},(2 \ell-1) \frac{\pi}{4}$, with $\ell=4,5, \ldots$ The non-negative poles, in turn, are 0 (which is a pole of multiplicity $2), \frac{3 \pi}{8}, \pi, \frac{3 \pi}{2}, \ell \frac{\pi}{2}$, where $\ell=4,5, \ldots$ Moreover, thanks to $(89),\left(\psi_{3}(s)\right)^{-1}$ possesses an expansion similar to $(92):$

$$
\begin{aligned}
\left(\psi_{3}(s)\right)^{-1} & =\frac{\pi}{2 i\left(s-i \frac{\pi}{4}\right)} \prod_{k \in \mathbb{Z} \backslash\{0, \pm 1\}}\left(1+\frac{2 s}{i \pi k}\right)\left(1+\frac{4 s}{i(2 k-1) \pi}\right)^{-1}\left(1+\frac{2 s}{i \pi}\right)\left(1+\frac{4 s}{i \pi}\right)^{-1} \\
& \times\left(1-\frac{2 s}{i \pi}\right)\left(1-\frac{4 s}{3 i \pi}\right)^{-1}\left(1-\frac{2 s}{i \pi}\right)^{-1}\left(1+\frac{2 s}{i \pi}\right)^{-1} \frac{4}{\pi^{2}}\left(1-\frac{8 s}{3 i \pi}\right)\left(1+\frac{8 s}{3 i \pi}\right)\left(\frac{3 \pi}{8}\right)^{2} \\
& =\frac{9 \pi}{32 i\left(s-\frac{i \pi}{4}\right)} \prod_{k \in \mathbb{Z} \backslash\{0, \pm 1\}}\left(1+\frac{2 s}{i \pi k}\right)\left(1+\frac{4 s}{i(2 k-1) \pi}\right)^{-1}\left(1+\frac{8 s}{3 i \pi}\right)\left(1+\frac{4 s}{i \pi}\right)^{-1}\left(1-\frac{8 s}{3 i \pi}\right)\left(1-\frac{4 s}{3 i \pi}\right)^{-1}
\end{aligned}
$$

This gives the form of the expansion (90) for $h_{\psi_{3}}(z)$, and shows that $h_{\psi_{3}}(z)$ maps the upper half plane into the upper half plane, thanks to the Krein theorem. Hence $\psi_{3}(s)$ is passive.

As before, see Example 3.19, we conducted an experiment with the help of the 3-stage Radau IIA convolution quadrature method. The rest of the parameters of the experiments are provided in Table 7 . The results of the

\begin{tabular}{rcccccccc}
$L_{x}$ & $L_{y}$ & $L_{x}^{\sigma}$ & $L_{y}^{\sigma}$ & $\sigma_{x}(x)$ & $\sigma_{y}(y)$ & $\Delta x$ & $\Delta t$ & $f(t, x, y)$ \\
\hline 12 & 16 & 2 & 0 & $20 x^{2}$ & 0 & 0.05 & 0.05 & $\mathrm{e}^{-40 x^{2}-40 y^{2}-12(t-10)^{2}}\left(24^{2}(t-10)^{2}-24\right) H(2-|x|) H(2-|y|)$
\end{tabular}

TABlE 7. Parameters for the experiment with a dielectric permittivity and magnetic permeability (89). We discretize directly the problem (5) where the PML change of variables (32) is used in direction $x$. We use zero initial conditions and the right hand side (in the time domain) $f(t, x, y)$. By $H(x)$ we denote the left-continuous Heaviside function. The notation can be found in the beginning of Section 3.1.2.

experiment are shown in Figure 9. We can observe the non-causality of the behaviour of the solution norm, when the Bérenger's PML is used, see Example 3.19 for a possible explanation, as well as its growth (the largest achieved value of $\left\|H_{z}\right\|$ not shown in the plot was close to $2 \cdot 10^{7}$ ), which evidences the instability of the PML. On the other hand, both PMLs (32) with $\psi(s)=\psi_{2}(s)$ and $\psi(s)=\psi_{3}(s)$ are stable, however, one can observe distinctively a small difference in their behaviour for this problem in the rightmost plot of Figure 9, contrary to what happens in Example 5.23, Figure 7; notice that the difference between the computed norms is fairly small. We measured the norms inside the domain (i.e. without the PML), and the difference between them is much less pronounced (it would not be visible on the same scale as in Figure 9).

Moreover, we can see that the solution norm in this case decreases very slowly, which is probably due to the dispersivity of this problem (we checked that this decay does not come from a slight dissipation of the RungeKutta method by computing the norm of the solution obtained with twice smaller time step; the difference between the norms is negligible). The stability of the PML (32) with $\psi(s)=\psi_{2,3}(s)$ is a numerical illustration of the possibility of the extension of the results of Theorem 5.12 to a slightly more general class of materials. 

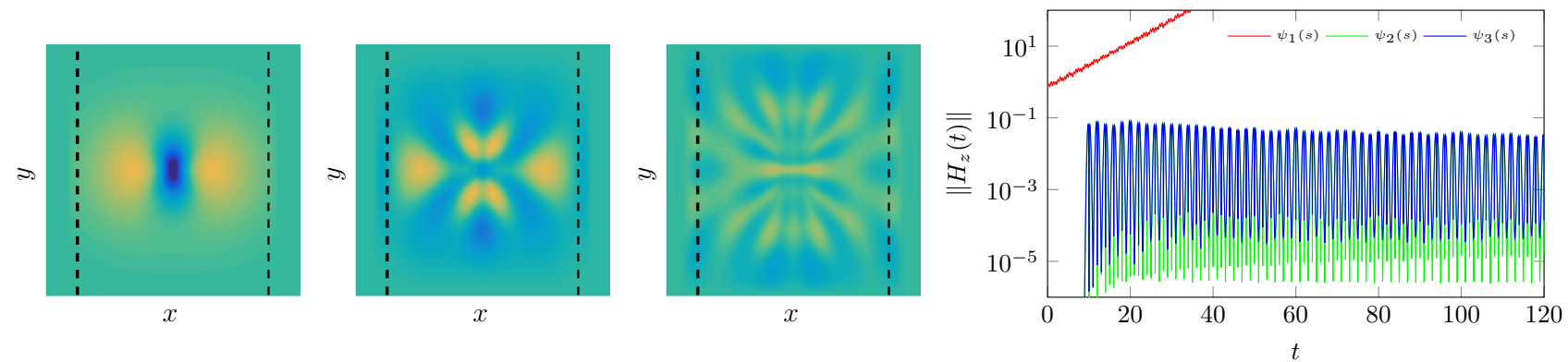

Figure 9. From left to right: a solution $H_{z}$ of Example 5.25 computed with the new PML with $\psi(s)=\psi_{2}(s)$, see (89), at the times $t=15.75,31.75$ and 59.9 ; the norm of the solution $H_{z}$ (inside the physical domain and the perfectly matched layer) with respect to time computed with different PMLs. The boundary between the domain and the perfectly matched layer is marked in black.

\section{Conclusions and Open Questions}

\subsection{Open Questions: Stable PMLs in 3D}

It is natural to ask whether the suggested PML technique and the analysis can be extended to a more broad class of problems, in particular, to the wave propagation in 3D. Naively, one would expect that in the simplest case of the Maxwell equations with a diagonal tensor of the dielectric permittivity and $\mu(s)=1$, it is sufficient to study the well-posedness of the PML (39) applied to the acoustic dispersive 3D wave equation (written in the Laplace domain)

$$
a(s) \partial_{x}^{2} u+b(s) \partial_{y}^{2} u+c(s) \partial_{z}^{2} u-s^{2} d(s) u=0 .
$$

This is, however, not the case. One should rather consider the vector equation

$$
\operatorname{curl} \operatorname{curl} \mathbf{E}+s^{2} \underline{\underline{\varepsilon}}(s) \mathbf{E}=0,
$$

with $\underline{\underline{\varepsilon}}$ being a diagonal matrix. This leads to several difficulties, both analytic and conceptual. First of all, when $\underline{\underline{\varepsilon}}=\mathrm{Id}$, it is well-known that the standard PMLs are stable. However, proving the coercivity of the corresponding sesquilinear form with the PML in $\mathbb{C}_{+}$, as we have done in $2 \mathrm{D}$, is no longer trivial, because the corresponding spatial operator looses its self-adjoint nature. On the other hand, it is still possible to prove very special inf-sup conditions. This is a subject of future research.

Second, it is not always possible to stabilize the PML for 3D dispersive problems using the change of variables as in this article. In particular, in [7], one considers the 3D wave propagation in cold strongly magnetized plasmas. There $\underline{\underline{\varepsilon}}(s)=\operatorname{diag}\left(1,1,1+\frac{\omega_{p}^{2}}{s^{2}}\right)$, with $\omega_{p} \in \mathbb{R}$ being a plasma frequency, and $\mu(s)=1$. However, even in this very simple case the system cannot be reduced to the form (93). Moreover, one can demonstrate that the dispersion relation is not of the form

$$
a(s) k_{x}^{2}+b(s) k_{y}^{2}+c(s) k_{z}^{2}+s^{2} d(s)=0,
$$

but is a product of two such terms. The first term corresponds to isotropic non-dispersive waves (i.e. $a=$ $b=c=d=1$ in (94)), which are absorbed by the classical PMLs (i.e. the choice $\psi(s)=1$ would be stable). Whereas the second term is of the form (94) with $a(s)=b(s)=\left(1+\frac{\omega_{p}^{2}}{s^{2}}\right)^{-1}$ and $c(s)=d(s)=1$, for which the 
choice $\psi(s)=a(s)=\left(1+\frac{\omega_{p}^{2}}{s^{2}}\right)^{-1}$ is stable. Nevertheless, since both kinds of waves are present simultaneously for the same frequency, none of these choices is suitable. It is possible to show that there exists no $\psi(s)$ satisfying assumptions of Section 5 that would lead to a stable 3D PML system in this case. The construction of stable PMLs for cold plasmas requires a more elaborate treatment, and constitutes the subject of [7]. However, the method of this article serves as an important component of the technique suggested in [7].

To summarize, the new PML change of variables (39) does not provide a general method for the stabilization of the PMLs for a 3D anisotropic dispersive Maxwell system, however, serves as a component for stabilizing the PMLs for such systems.

\subsection{Conclusions}

In this work we have shown how to construct stable perfectly matched layers for a class of anisotropic dispersive models described in the Laplace domain by the wave equation with frequency-dependent coefficients

$$
a(s) \partial_{x x} u+b(s) \partial_{y y} u-s^{2} c(s)=0, s \in \mathbb{C}_{+},
$$

where the coefficients $a(s), b(s), c(s)$ are analytic in $\mathbb{C}_{+}$, and satisfy $\operatorname{Re}(\bar{s} a(s))>0, \operatorname{Re}(\bar{s} b(s))>0, \operatorname{Re}(s c(s))>$ 0 , for $s \in \mathbb{C}_{+}$. Following [9], in order to construct the PML in one direction (assuming the PML layer is located in a half-plane $x>0$ ), we suggest to use the following change of variables:

$$
x \rightarrow x+\frac{\psi(s)}{s} \int_{0}^{x} \sigma\left(x^{\prime}\right) d x^{\prime}, x>0,
$$

where $\psi(s)$ satisfies $\operatorname{Re}(\bar{s} \psi(s))>0, s \in \mathbb{C}_{+}$. This article provides choices of $\psi(s)$ that would stabilize the PMLs. Based on the Laplace domain analysis in the free space for $\sigma=$ const, we claim that the following choices of $\psi(s)$ would result in stable PMLs in the $x$-direction:

(1) for arbitrary passive models: $\psi(s)=a(s)$;

(2) for isotropic passive models (where $a(s)=b(s)): \psi(s)=\left(\alpha c(s)+(1-\alpha) a^{-1}(s)\right)^{-1}, 0 \leq \alpha \leq 1$;

(3) in the case when $a(s)^{-1}, b(s)^{-1}, c(s)$ correspond to generalized Lorentz models, one can choose $\psi(s)$, s.t. $\psi(s)^{-1}$ is Lorentz and which would satisfy the following condition for all $\omega \in \mathbb{R}$ :

$$
\tilde{a}(\omega) \tilde{b}(\omega)<0 \text { or } \tilde{a}(\omega) \tilde{c}(\omega)>0 \Longrightarrow \tilde{a}(\omega) \tilde{\psi}(\omega) \geq 0 .
$$

Moreover, if $b(s)=c(s)^{-1}$ (2D uniaxial cold plasma model (8)), the only possible choice among passive $\psi(s)^{-1}$ satisfying Assumption 4.1 is $\psi(s)=a(s)$.

We confirm the obtained results with the help of numerical experiments, including examples with dissipation. Indeed, there are many open questions remaining which are the subject of the future work. In particular, they include the construction of stable PMLs for 3D anisotropic Maxwell's equations, even in the simplest case of a diagonal tensor of dielectric permittivity (where, as we discussed earlier, the instabilities cannot be overcome by the use of a special frequency-dependent change of variables). Another important question is a construction of stable PMLs for nonpassive materials, which is also a subject of the future research. 


\section{Appendix A. Proof of Lemma 2.2}

Define $z=i s, s \in \mathbb{C}_{+}$, and recall that $f_{c}(z):=z c(-i z)$ is a Herglotz function. The upper bound on $|f(z)|$ can be deduced e.g. from [28, Theorem 8', p. 18]. For consistency we show another way of computing it, using the methods of [60], which will serve as a basis to compute the lower bound for $\operatorname{Im} f_{c}(z)$ as well.

The main idea is to construct a function on the unit circle to which the Schwarz's lemma [60, Chapter 4, Theorem 1] can be easily applied. Let us set $h(s)=s c(s)$. Taking $s_{0} \in \mathbb{C}_{+}$, we define the Moebius transformation $[60, \mathrm{p} .43,44]$

$$
\begin{aligned}
r & :=\frac{s-s_{0}}{s+\overline{s_{0}}}, \quad|r|<1, s \in \mathbb{C}_{+}, \\
g\left(\frac{s-s_{0}}{s+\overline{s_{0}}}\right) & :=\frac{h(s)-h\left(s_{0}\right)}{h(s)+\overline{h\left(s_{0}\right)}} .
\end{aligned}
$$

The function $g(r)$ is analytic inside the unit circle $|r|<1 ; g(0)=0$ and $|g(r)|<1$. Then, thanks to the Schwarz's lemma, $|g(r)| \leq|r|$ inside the unit circle. Hence,

$$
\left|\frac{h(s)-h\left(s_{0}\right)}{h(s)+\overline{h\left(s_{0}\right)}}\right| \leq\left|\frac{s-s_{0}}{s+\overline{s_{0}}}\right| .
$$

(1) The proof of the upper bound on $|h(s)|$. From (95) it follows

$$
\left|h(s) h\left(s_{0}\right)^{-1}-1\right| \leq\left|\frac{s-s_{0}}{s+\overline{s_{0}}}\right|\left|h(s) h\left(s_{0}\right)^{-1}-\overline{h\left(s_{0}\right)} h\left(s_{0}\right)^{-1}\right| \leq\left|\frac{s-s_{0}}{s+\overline{s_{0}}}\right|\left(\left|h(s) h\left(s_{0}\right)^{-1}\right|+1\right) .
$$

Using $\left|h(s) h\left(s_{0}\right)^{-1}-1\right| \geq\left|h(s) h\left(s_{0}\right)\right|^{-1}-1$, and recalling that $r=\left|\frac{s-s_{0}}{s+s_{0}}\right|$, and $|r|<1$, we get the following bound

$$
|h(s)| \leq\left|h\left(s_{0}\right)\right|(1+r)(1-r)^{-1}=\left|h\left(s_{0}\right)\right|(1+r)^{2}\left(1-r^{2}\right)^{-1} \leq 4\left|h\left(s_{0}\right)\right|\left(1-r^{2}\right)^{-1} .
$$

Notice that the function $\left(1-r^{2}\right)^{-1}$ grows in $r$; hence, let us obtain the upper bound on $r$ in terms of $\operatorname{Re} s$ and $|s|$ :

$$
\left|\frac{s-s_{0}}{s+\overline{s_{0}}}\right|^{2}=\frac{|s|^{2}-2 \operatorname{Re}\left(s \overline{s_{0}}\right)+\left|s_{0}\right|^{2}}{|s|^{2}+2 \operatorname{Re}\left(s s_{0}\right)+\left|s_{0}\right|^{2}}=1-\frac{2 \operatorname{Re}\left(s s_{0}\right)+2 \operatorname{Re}\left(s \overline{s_{0}}\right)}{|s|^{2}+2 \operatorname{Re}\left(s s_{0}\right)+\left|s_{0}\right|^{2}}=1-\frac{4 \operatorname{Re} s \operatorname{Re} s_{0}}{|s|^{2}+2 \operatorname{Re}\left(s s_{0}\right)+\left|s_{0}\right|^{2}} .
$$

The lower bound for

$$
\frac{\operatorname{Re} s \operatorname{Re} s_{0}}{\left|s+\overline{s_{0}}\right|^{2}} \geq \frac{1}{2} \frac{\operatorname{Re} s \operatorname{Re} s_{0}}{|s|^{2}+\left|s_{0}\right|^{2}}
$$

Therefore, using (96), we obtain

$$
|h(s)| \leq 2\left|h\left(s_{0}\right)\right|\left(\operatorname{Re} s \operatorname{Re} s_{0}\right)^{-1}\left(|s|^{2}+\left|s_{0}\right|^{2}\right) \leq C^{\prime}\left(|s|^{2}+\left|s_{0}\right|^{2}\right)(\operatorname{Re} s)^{-1},
$$

for some $C^{\prime}>0$. From this we immediately obtain, for some $C>0$,

$$
|s c(s)| \leq C^{\prime}|s|^{2}\left(1+\left|s_{0}\right|^{2}|s|^{-2}\right)(\operatorname{Re} s)^{-1} \leq C|s|^{2}(\operatorname{Re} s)^{-1} \max \left(1,(\operatorname{Re} s)^{-2}\right), s \in \mathbb{C}_{+} .
$$

The same (up to a constant) bound can be obtained with the help of [28, Theorem 8, p. 18]. 
(2) The proof of the lower bound on $\operatorname{Re} h(s), s \in \mathbb{C}_{+}$. We rewrite (95) as follows, with the use of (97):

$$
\frac{|h(s)|^{2}-2 \operatorname{Re}\left(\overline{h\left(s_{0}\right)} h(s)\right)+\left|h\left(s_{0}\right)\right|^{2}}{|h(s)|^{2}+2 \operatorname{Re}\left(h\left(s_{0}\right) h(s)\right)+\left|h\left(s_{0}\right)\right|^{2}}=1-\frac{4 \operatorname{Re} h(s) \operatorname{Re} h\left(s_{0}\right)}{\left|h(s)+\overline{h\left(s_{0}\right)}\right|^{2}} \leq 1-\frac{4 \operatorname{Re} s \operatorname{Re} s_{0}}{|s|^{2}+2 \operatorname{Re}\left(s_{0} s\right)+\left|s_{0}\right|^{2}} .
$$

This gives, with the use of (98),

$$
\frac{\operatorname{Re} h(s) \operatorname{Re} h\left(s_{0}\right)}{\left|h(s)+\overline{h\left(s_{0}\right)}\right|^{2}} \geq \frac{1}{2} \frac{\operatorname{Re} s \operatorname{Re} s_{0}}{|s|^{2}+\left|s_{0}\right|^{2}} .
$$

Next, notice that $\left|h(s)+\overline{h\left(s_{0}\right)}\right|^{2} \geq\left(\operatorname{Re} h(s)+\operatorname{Re} \overline{h\left(s_{0}\right)}\right)^{2} \geq\left(\operatorname{Re} h\left(s_{0}\right)\right)^{2} . \operatorname{Using}|s|^{2}+\left|s_{0}\right|^{2} \leq C_{0} \max \left(|s|^{2}, 1\right), C_{0}>$ 0 , we obtain the desired bound:

$$
\operatorname{Re} h(s) \geq C \min \left(|s|^{-2}, 1\right) \operatorname{Re} s \geq C|s|^{-2} \operatorname{Re} s \min \left(1,(\operatorname{Re} s)^{2}\right) .
$$




\section{Appendix B. Proof of Corollary 3.9}

First, let us prove the result for arbitrary materials. Notice that for any $a(s), \psi(s)$ satisfying the conditions of the corollary, the bound (42) holds with the following parameters, see Corollary 2.4,

$$
\left|\frac{\psi(s)}{a(s)}\right|=\left|\frac{\bar{s} \psi(s)}{\bar{s} a(s)}\right| \leq|s||\psi(s)|(\operatorname{Re}(\bar{s} a(s)))^{-1} \leq C|s|^{4}(\operatorname{Re} s)^{-2} \max \left(1,(\operatorname{Re} s)^{-4}\right), C>0 .
$$

For the Lorentz materials, let us bound $a(s)^{-1}$ which has an expansion (9), i.e. $a(s)^{-1}=1+\sum_{\ell=0}^{n_{a}} \frac{a_{\ell}}{s^{2}+\omega_{\ell}^{2}}$, with $a_{\ell}>0$ and $\omega_{\ell} \in \mathbb{R}$. Notice that for all $\omega \in \mathbb{R}$ and $s \in \mathbb{C}_{+}$, it holds that

$$
\left|\frac{1}{s^{2}+\omega^{2}}\right|=\frac{1}{|s-i \omega||s+i \omega|} \leq \frac{1}{|s| \operatorname{Re} s} .
$$

Then, for some $C, \tilde{C}>0$, the modulus $\left|a(s)^{-1}\right| \leq 1+\frac{C}{|s| \operatorname{Re} s} \leq \tilde{C} \max \left(1,(\operatorname{Re} s)^{-2}\right)$. Thanks to the inequality $\operatorname{Re}\left(s \psi(s)^{-1}\right)>\operatorname{Re} s$, cf. Theorem 4.4, we obtain

$$
\left|\frac{\psi(s)}{a(s)}\right| \leq\left|\frac{s a(s)^{-1}}{s \psi(s)^{-1}}\right| \leq \tilde{C}|s| \max \left(1,(\operatorname{Re} s)^{-2}\right)\left(\operatorname{Re} s \psi(s)^{-1}\right)^{-1} \leq \tilde{C}|s| \max \left(1,(\operatorname{Re} s)^{-3}\right) .
$$




\section{Appendix C. Construction of a Function $\psi(s)$ Satisfying Conditions of THEOREM 5.12}

In [9], the authors have proposed a procedure to construct the function $\psi(s)$ that results in stable perfectly matched layer for isotropic dispersive systems, based on a result similar to Theorem 5.12. Let us briefly comment on the extension (and a minor modification) of their procedure to anisotropic dispersive systems in question. Consider a passive sesquilinear form $A(u, v)$ that satisfies Assumption 5.1. Let us describe a procedure of choosing a function $\psi(s)$ for the PML change of variables that satisfies conditions of Theorem 5.12.

The condition (NSC1) of Theorem 5.12 can be rewritten alternatively in the following form. Either the sign of $\tilde{\psi}$ is constant on the real axis (and thus is positive, since $\tilde{\psi}(\omega) \rightarrow 1$ as $\omega \rightarrow \infty$ ), or there exists a set of intervals $\mathcal{I}_{k}=\left(\omega_{2 k-1}, \omega_{2 k}\right), k=1, \ldots, m$, with $0 \leq \omega_{1}<\omega_{2} \leq \omega_{3}<\ldots \leq \omega_{2 k-1}<\omega_{2 m}=+\infty$, s.t. the sign of the function $\tilde{\psi}(\omega)$ on an interval $\mathcal{I}_{k}$ is opposite to the sign of $\tilde{\psi}(\omega)$ on the intervals $\mathcal{I}_{k-1}$ and $\mathcal{I}_{k+1}$. These signs are defined by the signs of $\tilde{a}(\omega)$. Let us remark that $\omega_{2 m}=+\infty$, since as $\omega \rightarrow+\infty, \tilde{c}(\omega) \tilde{a}(\omega)>0$, and thus, $\tilde{\psi}(\omega)>0$.

The case when the sign of $\tilde{\psi}$ is constant on $\mathbb{R}$, is trivially reduced to using the Bérenger's PML $\tilde{\psi}=1$. Otherwise, following [9], we consider two cases:

(1) $m=2 \ell-1, \ell>1$. In this case the sign of $\tilde{\psi}(\omega)$ on $\mathcal{I}_{1}$ coincides with the sign of $\tilde{\psi}(\omega)$ on $\left(\omega_{2 m},+\infty\right)$, i.e. is positive. One can verify that the following holds true:

$$
\begin{aligned}
\omega \in\left(\omega_{4 k-3}, \omega_{4 k-2}\right) & \Longrightarrow \tilde{\psi}(\omega) \geq 0, \\
\omega \in\left(\omega_{4 k-1}, \omega_{4 k}\right) & \Longrightarrow \tilde{\psi}(\omega) \leq 0 .
\end{aligned}
$$

Thus, one of the choices of $\tilde{\psi}(\omega)$ is given by

$$
\tilde{\psi}(\omega)=\prod_{k=1}^{\ell-1} \frac{\left(\omega^{2}-r_{k}^{2}\right)}{\left(\omega^{2}-p_{k}^{2}\right)}, \quad r_{k} \in\left[\omega_{4 k-2}, \omega_{4 k-1}\right], p_{k} \in\left[\omega_{4 k}, \omega_{4 k+1}\right]
$$

First of all, notice that for all $k=1, \ldots, \ell-2$, the following interlacing property holds true: $r_{k} \leq \omega_{4 k-1}<$ $\omega_{4 k} \leq p_{k} \leq \omega_{4 k+1}<\omega_{4 k+2} \leq r_{k+1}$. Applying Lemma 4.10, we see that $\tilde{\psi}(\omega)$ satisfies $\operatorname{Re}(\bar{s} \psi(s))>0$ in $\mathbb{C}_{+}$. It remains only to verify that $\tilde{\psi}$ defined as above satisfies the sign conditions (108). Taking $\omega_{*} \in\left(\omega_{4 j-3}, \omega_{4 j-2}\right)$, we can split

$$
\tilde{\psi}(\omega)=\prod_{k=1}^{j-1} \frac{\left(\omega^{2}-r_{k}^{2}\right)}{\left(\omega^{2}-p_{k}^{2}\right)} \prod_{k=j}^{\ell-2} \frac{\left(\omega^{2}-r_{k}^{2}\right)}{\left(\omega^{2}-p_{k}^{2}\right)}=\tilde{\psi}_{j}^{(1)}(\omega) \tilde{\psi}_{j}^{(2)}(\omega) .
$$

One sees that all the poles and zeros of $\tilde{\psi}_{j}^{(1)}(\omega)$ are smaller than $\omega_{*}$ (notice that $p_{j-1} \leq \omega_{4 j-3}$ ), hence $\tilde{\psi}_{j}^{(1)}\left(\omega_{*}\right)>0$. Similarly, all the poles and zeros of $\tilde{\psi}_{j}^{(2)}(\omega)$ are larger than $\omega_{*}$ (since $r_{j} \geq \omega_{4 j-2}$ ), hence by direct computation $\tilde{\psi}_{j}^{(2)}\left(\omega_{*}\right)>0$. Therefore, $\tilde{\psi}\left(\omega_{*}\right)>0$.

Similarly one can verify the validity of $(108)$ for $\omega_{*} \in\left(\omega_{4 j-1}, \omega_{4 j}\right)$.

(2) $m=2 \ell, \ell>1$. In this case the sign of $\tilde{\psi}(\omega)$ on $\mathcal{I}_{1}$ is negative. One can verify that the following holds true:

$$
\begin{aligned}
\omega \in\left(\omega_{4 k-3}, \omega_{4 k-2}\right) & \Longrightarrow \tilde{\psi}(\omega) \leq 0, \\
\omega \in\left(\omega_{4 k-1}, \omega_{4 k}\right) & \Longrightarrow \tilde{\psi}(\omega) \geq 0 .
\end{aligned}
$$


One of the choices of $\tilde{\psi}(\omega)$ is provided by

$$
\tilde{\psi}(\omega)=\prod_{k=1}^{\ell-1} \frac{\left(\omega^{2}-r_{k}^{2}\right)}{\left(\omega^{2}-p_{k}^{2}\right)}, \quad r_{1} \in\left[0, \omega_{1}\right], r_{k} \in\left[\omega_{4 k}, \omega_{4 k+1}\right], p_{1} \in\left[\omega_{2}, \omega_{3}\right], p_{k} \in\left[\omega_{4 k+2}, \omega_{4 k+3}\right] .
$$

As before, the condition $\operatorname{Re}(\bar{s} \psi(s))>0$ for $s \in \mathbb{C}_{+}$, and the sign conditions (109) can be ensured by the direct use of Lemma 4.10 and computations as in the previous case. 


\section{Appendix D. Proof of Equivalence in Lemma 5.16}

Lemma D.1. Let $a(s), \psi(s), c(s)$ satisfy Assumption 5.11. Assume (NSC2)-(a) holds true, i.e. $c(s) a(s)^{-1} \psi(s)$ is passive. Then (NSC1)-(a) holds, i.e. for all $\omega \in \Omega_{p}^{a c>0}$ the product $\tilde{\psi}(\omega) \tilde{a}(\omega) \geq 0$.

Proof. First of all, in $\omega=0$, due to Theorem 4.5 , it holds that $\tilde{\psi}(0) \tilde{a}(0) \geq 0$. Due to the equivalence of the positivity of the derivative $(\omega \tilde{f}(\omega))^{\prime}>0, \omega \in \mathbb{R}$, and the property $\operatorname{Re}(s f(s))>0$ in $\mathbb{C}_{+}$, the following derivative is strictly positive for $\omega \in \mathbb{R}$ with the exception of poles of $\tilde{c} \tilde{\psi} \tilde{a}^{-1}$ :

$$
\left(\omega \tilde{c}(\omega) \tilde{\psi}(\omega) \tilde{a}(\omega)^{-1}\right)^{\prime}=(\omega \tilde{c}(\omega))^{\prime} \tilde{\psi}(\omega) \tilde{a}(\omega)^{-1}+\omega \tilde{c}(\omega) \tilde{\psi}(\omega)^{\prime} \tilde{a}(\omega)^{-1}+\omega \tilde{c}(\omega) \tilde{\psi}(\omega)\left(\tilde{a}(\omega)^{-1}\right)^{\prime}>0
$$

Take $\omega$ s.t. $\tilde{c}(\omega) \tilde{a}(\omega)>0$ and assume that $\tilde{a}(\omega) \tilde{\psi}(\omega)<0$. Using the equation (14), which shows the passivity of $a(s)^{-1}$, and Lemmas 4.8 and 4.9 about the signs of the derivatives of the passive functions, we obtain that each term of the above is strictly negative, and thus the contradiction.

Remark D.2. One could try proving Lemma 5.16 by examining the sign of the derivative (102); however, when $\tilde{c}(\omega) \tilde{a}(\omega)<0$, and $\tilde{\psi}(\omega) \tilde{a}(\omega)$ is of any sign, one does not see immediately that the expression (102) is strictly positive. 


\section{Appendix E. Dispersion Relation for Anisotropic Lorentz Materials}

In this section we provide some details on a computation of the dispersion relation of the Lorentz system (60). For simplicity, we will compute it for the corresponding 1D formulation (in the 2D case the computation remains the same):

$$
\begin{array}{r}
\partial_{t} E_{x}+\sum_{\ell=0}^{n_{x}} \varepsilon_{x \ell} j_{x \ell}=\partial_{y} H_{z}, \\
\partial_{t} H_{z}+\sum_{\ell=0}^{n_{\mu}} \mu_{\ell} j_{\mu \ell}=\partial_{y} E_{x}, \\
\partial_{t} j_{x \ell}+\omega_{x \ell}^{2} p_{x \ell}=E_{x}, \quad \partial_{t} p_{x \ell}=j_{x \ell}, \quad \ell=0, \ldots, n_{x}, \\
\partial_{t} j_{\mu \ell}+\omega_{\mu \ell}^{2} p_{\mu \ell}=H_{z}, \quad \partial_{t} p_{\mu \ell}=j_{\mu \ell}, \quad \ell=0, \ldots, n_{\mu} .
\end{array}
$$

We consider the plane-wave solutions of the above system in the form $\mathbf{V} \mathrm{e}^{-i \omega t+i \mathbf{k} \cdot \mathbf{x}}$, where $\mathbf{V} \in \mathbb{C}^{2\left(n_{x}+1\right)+2\left(n_{\mu}+1\right)+2}$ is a constant vector. There exists such a solution with $\mathbf{V} \neq 0$ if and only if the determinant of the following block matrix vanishes:

$$
\begin{aligned}
& U=\left(\begin{array}{ll}
U_{E_{x} E_{x}} & U_{E_{x} H_{z}} \\
U_{H_{z} E_{x}} & U_{H_{z} H_{z}}
\end{array}\right), \quad U_{E_{x} H_{z}}=\operatorname{diag}\left(-i k_{y}, 0, \ldots, 0\right) \in \mathbb{C}^{\left(2 n_{x}+1\right) \times\left(2 n_{\mu}+1\right)}, \\
& U_{H_{z} E_{x}}=\operatorname{diag}\left(-i k_{y}, 0, \ldots, 0\right) \in \mathbb{C}^{\left(2 n_{x}+1\right) \times\left(2 n_{\mu}+1\right)}, \\
& U_{E_{x} E_{x}}=\left(\begin{array}{cccccccc}
-i \omega & \varepsilon_{x 0} & 0 & \varepsilon_{x 1} & 0 & \ldots & \varepsilon_{x n_{x}} & 0 \\
\mathbf{v}_{2\left(n_{x}+1\right) \times 1} & & \multicolumn{8}{c}{\Omega_{x}} & & &
\end{array}\right) \text {, } \\
& U_{H_{z} H_{z}}=\left(\begin{array}{cccccccc}
-i \omega & \mu_{0} & 0 & \mu_{1} & 0 & \ldots & \mu_{n_{\mu}} & 0 \\
\mathbf{v}_{2\left(n_{\mu}+1\right) \times 1} & \multicolumn{8}{c}{\Omega_{y}} & &
\end{array}\right) \text {, }
\end{aligned}
$$

where

$$
\begin{aligned}
& \Omega_{\alpha}=\left(\begin{array}{ccccccc}
-i \omega & \omega_{\alpha 0}^{2} & 0 & 0 & \ldots & 0 & 0 \\
-1 & -i \omega & 0 & 0 & \ldots & 0 & 0 \\
0 & 0 & -i \omega & \omega_{\alpha 1}^{2} & \ldots & 0 & 0 \\
0 & 0 & -1 & -i \omega & \ldots & 0 & 0 \\
\vdots & & & & & & \\
0 & 0 & 0 & 0 & \ldots & -i \omega & \omega_{\alpha n_{\alpha}}^{2} \\
0 & 0 & 0 & 0 & \ldots & -1 & -i \omega
\end{array}\right), \alpha \in\{x, \mu\} \\
& \mathbf{v}_{2 m \times 1} \text { is a vector of size } 2 m \times 1, \quad\left(\mathbf{v}_{2 m \times 1}\right)_{k}= \begin{cases}-1 & k \text { is odd, } \\
0 & k \text { is even. }\end{cases}
\end{aligned}
$$

To compute the determinant of $U$, we multiply each of its $2(k+1)+1$ st column, with $k=0, \ldots, n_{x}$, by $(-i \omega)^{-1}$ and add it to the $2(k+1)$ st column. Next, we repeat the same procedure for $2\left(n_{x}+1\right)+4+2 m$ and 
$2\left(n_{x}+1\right)+3+2 m$ th columns correspondingly, where $m=0, \ldots, n_{\mu}$. This results in the following expression for the determinant of the matrix $U$ :

$$
\begin{aligned}
& \operatorname{det} U=\operatorname{det}\left(\begin{array}{cc}
\tilde{U}_{E_{x} E_{x}} & U_{E_{x} H_{z}} \\
U_{H_{z} E_{x}} & \tilde{U}_{H_{z} H_{z}}
\end{array}\right) \\
& \tilde{U}_{E_{x} E_{x}}=\left(\begin{array}{cccccccc}
-i \omega & \varepsilon_{x 0} & 0 & \varepsilon_{x 1} & 0 & \ldots & \varepsilon_{x n_{x}} & 0 \\
\mathbf{v}_{2\left(n_{x}+1\right) \times 1} & & & & \tilde{\Omega}_{x} & & &
\end{array}\right), \\
& \tilde{U}_{H_{z} H_{z}}=\left(\begin{array}{cccccccc}
-i \omega & \mu_{0} & 0 & \mu_{1} & 0 & \ldots & \mu_{n_{\mu}} & 0 \\
\mathbf{v}_{2\left(n_{y}+1\right) \times 1} & & & & \tilde{\Omega}_{y} & & &
\end{array}\right) \text {, }
\end{aligned}
$$

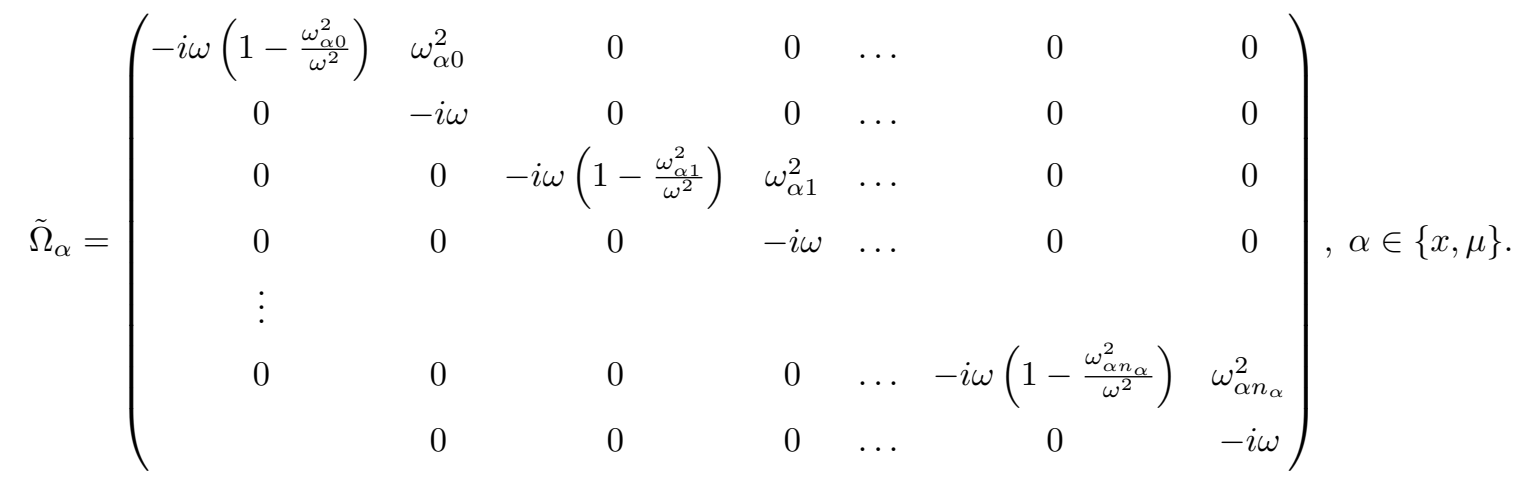

This, in turn can be rewritten as

$$
\begin{aligned}
& |\operatorname{det} U|=\left|(-i \omega)^{n_{x}+n_{\mu}+2}\right| \operatorname{det}\left(\begin{array}{cc}
\tilde{U}_{E_{x} E_{x}}^{\prime} & \tilde{U}_{E_{x} H_{z}}^{\prime} \\
\tilde{U}_{H_{z} E_{x}}^{\prime} & \tilde{U}_{H_{z} H_{z}}^{\prime}
\end{array}\right) \mid \\
& \tilde{U}_{E_{x} E_{x}}^{\prime}=\left(\begin{array}{ccccc}
-i \omega & \varepsilon_{x 0} & \varepsilon_{x 1} & \ldots & \varepsilon_{x n_{x}} \\
-\mathbf{1}_{\left(n_{x}+1\right) \times 1} & & \tilde{\Omega}_{x}^{\prime} &
\end{array}\right), \quad \tilde{U}_{H_{z} H_{z}}^{\prime}=\left(\begin{array}{ccccc}
-i \omega & \mu_{0} & \mu_{1} & \ldots & \mu_{n_{\mu}} \\
-\mathbf{1}_{\left(n_{\mu}+1\right) \times 1} & & \tilde{\Omega}_{y}^{\prime} &
\end{array}\right),
\end{aligned}
$$

where

$$
\begin{aligned}
& \tilde{\Omega}_{\alpha}^{\prime}=\left(\begin{array}{cccc}
-i \omega\left(1-\frac{\omega_{\alpha 0}^{2}}{\omega^{2}}\right) & 0 & \ldots & 0 \\
0 & -i \omega\left(1-\frac{\omega_{x 1}^{2}}{\omega^{2}}\right) & \ldots & 0 \\
\vdots & 0 & \ldots & -i \omega\left(1-\frac{\omega_{x n_{x}}^{2}}{\omega^{2}}\right)
\end{array}\right), \alpha \in\{x, \mu\}, \\
& \mathbf{1}_{m \times 1} \text { is a vector of size } m \times 1, \quad \mathbf{1}_{m \times 1}=(1, \ldots, 1)^{T},
\end{aligned}
$$

and $\tilde{U}_{E_{x} H_{z}}^{\prime} \in \mathbb{C}^{\left(n_{x}+2\right) \times\left(n_{\mu}+2\right)}, \tilde{U}_{H_{z} E_{x}}^{\prime} \in \mathbb{C}^{\left(n_{\mu}+2\right) \times\left(n_{x}+2\right)}$ are such that $\left(\tilde{U}_{E_{x} H_{z}}^{\prime}\right)_{11}=\left(\tilde{U}_{H_{z} E_{x}}^{\prime}\right)_{11}=-i k_{y}$. Next, for $k=0, \ldots, n_{x}$, scale $k+1$ st row by $-\left(-i \omega\left(1-\frac{\omega_{x k}^{2}}{\omega^{2}}\right)\right)^{-1} \varepsilon_{x k}$ and add the result to the first row. Every $n_{x}+4+m$ th row, for $m=0, \ldots, n_{\mu}$ scaled by $-\left(-i \omega\left(1-\frac{\omega_{\mu m}^{2}}{\omega^{2}}\right)\right)^{-1} \mu_{m}$ we add to the $n_{x}+4$ th 
row. Notice that the entry $(1,1)$ of the matrix $\tilde{U}_{E_{x} E_{x}}^{\prime}$ becomes $-i \omega+\sum_{k=1}^{n_{x}+1}\left(-i \omega\left(1-\frac{\omega_{x k-1}^{2}}{\omega^{2}}\right)\right)^{-1} \varepsilon_{x k-1}=$ $-i \omega\left(1-\sum_{k=1}^{n_{x}+1}\left(\omega^{2}-\omega_{x k-1}^{2}\right)^{-1} \varepsilon_{x k-1}\right)=-i \omega \tilde{\varepsilon}_{1}(\omega)$. Similarly, the entry $(1,1)$ of the matrix $\tilde{U}_{H_{z} H_{z}}$ becomes $-i \omega \tilde{\mu}(\omega)$. After this procedure, it remains to compute the following:

$$
\begin{aligned}
& |\operatorname{det} U|=\left|(-i \omega)^{n_{x}+n_{\mu}+2}\right| \operatorname{det}\left(\begin{array}{cc}
\tilde{\tilde{U}}_{E_{x} E_{x}}^{\prime} & \tilde{U}_{E_{x} H_{z}}^{\prime} \\
\tilde{U}_{H_{z} E_{x}}^{\prime} & \tilde{\tilde{U}}_{H_{z} H_{z}}^{\prime}
\end{array}\right) \mid \\
& \tilde{\tilde{U}}_{E_{x} E_{x}}^{\prime}=\left(\begin{array}{ccccc}
-i \omega \tilde{\varepsilon}_{1}(\omega) & 0 & 0 & \ldots & 0 \\
-\mathbf{1}_{\left(n_{x}+1\right) \times 1} & & \tilde{\Omega}_{x}^{\prime} &
\end{array}\right), \quad \tilde{\tilde{U}}_{H_{z} H_{z}}^{\prime}=\left(\begin{array}{ccccc}
-i \omega \tilde{\mu}(\omega) & 0 & 0 & \ldots & 0 \\
-\mathbf{1}_{\left(n_{\mu}+1\right) \times 1} & & \tilde{\Omega}_{\mu}^{\prime} &
\end{array}\right),
\end{aligned}
$$

from which it immediately follows that

$$
\begin{aligned}
|\operatorname{det} U| & =\left|\omega^{n_{x}+n_{\mu}+2} \omega^{n_{x}+n_{\mu}+2} \prod_{\ell=0}^{n_{\mu}}\left(1-\frac{\omega_{\mu \ell}^{2}}{\omega^{2}}\right) \prod_{\ell=0}^{n_{x}}\left(1-\frac{\omega_{x \ell}^{2}}{\omega^{2}}\right)\right| \times\left|\operatorname{det}\left(\begin{array}{cc}
-i \omega \tilde{\varepsilon}_{1}(\omega) & -i k_{y} \\
-i k_{y} & -i \omega \tilde{\mu}(\omega)
\end{array}\right)\right| \\
& =\left|\prod_{\ell=0}^{n_{\mu}}\left(\omega^{2}-\omega_{\mu \ell}^{2}\right) \prod_{\ell=0}^{n_{x}}\left(\omega^{2}-\omega_{x \ell}^{2}\right)\right| \times\left|-\omega^{2} \tilde{\varepsilon}_{1} \tilde{\mu}+k_{y}^{2}\right| \\
& =\left|\prod_{\ell=0}^{n_{\mu}}\left(\omega^{2}-\omega_{\mu \ell}^{2}\right) \prod_{\ell=0}^{n_{x}}\left(\omega^{2}-\omega_{x \ell}^{2}\right) \tilde{\varepsilon}_{1}\right| \times\left|-\omega^{2} \tilde{\mu}+k_{y}^{2} \tilde{\varepsilon}_{1}^{-1}\right|
\end{aligned}
$$

We can generalize the above result to two dimensions; the dispersion relation for the system (60) reads (an extra factor $\omega$ comes from $i \omega \tilde{\varepsilon}_{2}(\omega)$ in the corresponding matrix in the final computation (107):

$$
F(\omega, \mathbf{k})=\prod_{\ell=0}^{n_{\mu}}\left(\omega^{2}-\omega_{\mu \ell}^{2}\right) \prod_{\ell=0}^{n_{x}}\left(\omega^{2}-\omega_{x \ell}^{2}\right) \prod_{\ell=0}^{n_{y}}\left(\omega^{2}-\omega_{y \ell}^{2}\right) \tilde{\varepsilon}_{1}(\omega) \tilde{\varepsilon_{2}}(\omega) \omega\left(k_{x}^{2} \tilde{\varepsilon}_{2}^{-1}+k_{y}^{2} \tilde{\varepsilon}_{1}^{-1}-\omega^{2} \tilde{\mu}(\omega)\right)=0 .
$$




\section{Appendix F. Dispersion Relation for Anisotropic Lorentz Materials with the PML}

In this appendix we briefly comment on how to compute the dispersion relation for the system (62).

Let us for simplicity assume that $n_{x}=n_{y}=n_{\mu}=n_{\psi}=0$. Following the procedure of Appendix E, we require that the determinant of the following matrix vanishes:

$$
\operatorname{det} V=\operatorname{det}\left(\begin{array}{ccccc}
V_{E_{x} E_{x}} & V_{E_{x} E_{y}} & V_{E_{x} H_{z}} & V_{E_{x} E_{y}^{*}} & V_{E_{x} H_{z}^{*}} \\
V_{E_{y} E_{x}} & V_{E_{y} E_{y}} & V_{E_{y} H_{z}} & V_{E_{y} E_{y}^{*}} & V_{E_{y} H_{z}^{*}} \\
V_{H_{z} E_{x}} & V_{H_{z} E_{y}} & V_{H_{z} H_{z}} & V_{H_{z} E_{y}^{*}} & V_{H_{z} H_{z}^{*}} \\
V_{E_{y}^{*} E_{x}} & V_{E_{y}^{*} E_{y}} & V_{E_{y}^{*} H_{z}} & V_{E_{y}^{*} E_{y}^{*}} & V_{E_{y}^{*} H_{z}^{*}} \\
V_{H_{z}^{*} E_{x}} & V_{H_{z}^{*} E_{y}} & V_{H_{z}^{*} H_{z}} & V_{H_{z}^{*} E_{y}^{*}} & V_{H_{z}^{*} H_{z}^{*}}
\end{array}\right),
$$

where

$$
\begin{aligned}
V_{E_{\alpha} E_{\alpha}} & =\left(\begin{array}{ccc}
-i \omega & \varepsilon_{\alpha 0} & 0 \\
-1 & -i \omega & \omega_{\alpha 0}^{2} \\
0 & -1 & -i \omega
\end{array}\right), \alpha \in\{x, y\}, \quad V_{H_{z} H_{z}} \quad=\left(\begin{array}{ccc}
-i \omega & \varepsilon_{\mu 0} & 0 \\
-1 & -i \omega & \omega_{\mu 0}^{2} \\
0 & -1 & -i \omega
\end{array}\right), \\
V_{E_{y}^{*} E_{y}^{*}}=V_{H_{z}^{*} H_{z}^{*}} & =\left(\begin{array}{ccc}
-i \omega+\sigma & c_{\psi 0} & 0 \\
-1 & -i \omega & r_{\psi 0}^{2} \\
0 & -1 & -i \omega
\end{array}\right)
\end{aligned}
$$

and the following matrices are empty or diagonal with a non-vanishing entry $(1,1)$ (the size of these matrices can be deduced from the size of the diagonal matrices which are given in a more explicit manner further):

$V_{E_{x} E_{y}}=V_{E_{y} E_{x}}=0, \quad V_{E_{x} E_{y}^{*}}=V_{E_{y}^{*} E_{x}}=0, \quad V_{E_{x} H_{z}^{*}}=V_{H_{z}^{*} E_{x}}=0, \quad V_{E_{y} H_{z}^{*}}=V_{H_{z}^{*} E_{y}}=0$,

$V_{E_{x} H_{z}}=V_{H_{z} E_{x}}=\left(\begin{array}{ccc}-i k_{y} & 0 & 0 \\ 0 & 0 & 0 \\ 0 & 0 & 0\end{array}\right), \quad V_{E_{y} H_{z}}=V_{H_{z} E_{y}}=\left(\begin{array}{ccc}i k_{x} & 0 & 0 \\ 0 & 0 & 0 \\ 0 & 0 & 0\end{array}\right), \quad V_{E_{y} E_{y}^{*}}=V_{H_{z} H_{z}^{*}}=\left(\begin{array}{ccc}\sigma & 0 & 0 \\ 0 & 0 & 0 \\ 0 & 0 & 0\end{array}\right)$,

$V_{E_{y}^{*} E_{y}}=V_{H_{z}^{*} H_{z}}=0, \quad V_{E_{y}^{*} H_{z}}=V_{H_{z}^{*} E_{y}}=\left(\begin{array}{ccc}i k_{x} & 0 & 0 \\ 0 & 0 & 0 \\ 0 & 0 & 0\end{array}\right), \quad V_{E_{y}^{*} H_{z}^{*}}=V_{H_{z}^{*} E_{y}^{*}}=V_{H_{z} E_{y}^{*}}=V_{E_{y} H_{z}^{*}}=0$.

We perform the following operations (numbering of rows/lines starts with 1) 
(1) $3 k$ th row multiply by $(-i \omega)^{-1}$, add to the $3 k-1$ st row, for $k \leq 5$. This results in

$$
\operatorname{det} V=\operatorname{det} V^{(1)}=\operatorname{det}\left(\begin{array}{ccccc}
V_{E_{x} E_{x}}^{(1)} & V_{E_{x} E_{y}} & V_{E_{x} H_{z}} & V_{E_{x} E_{y}^{*}} & V_{E_{x} H_{z}^{*}} \\
V_{E_{y} E_{x}} & V_{E_{y} E_{y}}^{(1)} & V_{E_{y} H_{z}} & V_{E_{y} E_{y}^{*}} & V_{E_{y} H_{z}^{*}} \\
V_{H_{z} E_{x}} & V_{H_{z} E_{y}} & V_{H_{z} H_{z}}^{(1)} & V_{H_{z} E_{y}^{*}} & V_{H_{z} H_{z}^{*}} \\
V_{E_{y}^{*} E_{x}} & V_{E_{y}^{*} E_{y}} & V_{E_{y}^{*} H_{z}} & V_{E_{y}^{*} E_{y}^{*}}^{(1)} & V_{E_{y}^{*} H_{z}^{*}} \\
V_{H_{z}^{*} E_{x}} & V_{H_{z}^{*} E_{y}} & V_{H_{z}^{*} H_{z}} & V_{H_{z}^{*} E_{y}^{*}} & V_{H_{z}^{*} H_{z}^{*}}^{(1)}
\end{array}\right),
$$

where

$$
\begin{aligned}
V_{E_{\alpha} E_{\alpha}}^{(1)}= & \left(\begin{array}{ccc}
-i \omega & \varepsilon_{\alpha 0} & 0 \\
-1 & -i \omega\left(1-\frac{\omega_{\alpha 0}^{2}}{\omega^{2}}\right) & \omega_{\alpha 0}^{2} \\
0 & 0 & -i \omega
\end{array}\right), \alpha \in\{x, y\}, \quad V_{H_{z} H_{z}}^{(1)}=\left(\begin{array}{ccc}
-i \omega & \varepsilon_{\mu 0} & 0 \\
-1 & -i \omega\left(1-\frac{\omega_{\mu 0}^{2}}{\omega^{2}}\right) & \omega_{\mu 0}^{2} \\
0 & 0 & -i \omega
\end{array}\right), \\
V_{E_{y}^{*} E_{y}^{*}}^{(1)}=V_{H_{z}^{*} H_{z}^{*}}^{(1)} & =\left(\begin{array}{ccc}
-i \omega+\sigma & c_{\psi 0} & 0 \\
-1 & -i \omega\left(1-\frac{r_{\psi 0}^{2}}{\omega^{2}}\right) & r_{\psi 0}^{2} \\
0 & 0 & -i \omega
\end{array}\right) .
\end{aligned}
$$

(2) for $k \leq 7$, multiply the $2 k$-th row by the element $\left(V_{2 k, 2 k}^{(1)}\right)^{-1}$, add to the $(2 k-1)$ st row. This results in the following

$$
\begin{aligned}
& \operatorname{det} V=\operatorname{det} V^{(2)}=\operatorname{det}\left(\begin{array}{ccccc}
V_{E_{x} E_{x}}^{(2)} & V_{E_{x} E_{y}} & V_{E_{x} H_{z}} & V_{E_{x} E_{y}^{*}} & V_{E_{x} H_{z}^{*}} \\
V_{E_{y} E_{x}} & V_{E_{y} E_{y}}^{(2)} & V_{E_{y} H_{z}} & V_{E_{y} E_{y}^{*}} & V_{E_{y} H_{z}^{*}} \\
V_{H_{z} E_{x}} & V_{H_{z} E_{y}} & V_{H_{z} H_{z}}^{(2)} & V_{H_{z} E_{y}^{*}} & V_{H_{z} H_{z}^{*}} \\
V_{E_{y}^{*} E_{x}} & V_{E_{y}^{*} E_{y}} & V_{E_{y}^{*} H_{z}} & V_{E_{y}^{*} E_{y}^{*}}^{(2)} & V_{E_{y}^{*} H_{z}^{*}} \\
V_{H_{z}^{*} E_{x}} & V_{H_{z}^{*} E_{y}} & V_{H_{z}^{*} H_{z}} & V_{H_{z}^{*} E_{y}^{*}} & V_{H_{z}^{*} H_{z}^{*}}^{(2)}
\end{array}\right), \\
& V_{E_{\alpha} E_{\alpha}}^{(2)}=\left(\begin{array}{ccc}
-i \omega \tilde{\varepsilon}_{\alpha}(\omega) & \varepsilon_{\alpha 0} & 0 \\
0 & -i \omega\left(1-\frac{\omega_{\alpha 0}^{2}}{\omega^{2}}\right) & \omega_{\alpha 0}^{2} \\
0 & 0 & -i \omega
\end{array}\right), \alpha \in\{x, y\}, \quad V_{H_{z} H_{z}}^{(2)}=\left(\begin{array}{ccc}
-i \omega \tilde{\mu}(\omega) & \varepsilon_{\mu 0} & 0 \\
0 & \left(1-\frac{\omega_{\mu 0}^{2}}{\omega^{2}}\right) & \omega_{\mu 0}^{2} \\
0 & 0 & -i \omega
\end{array}\right) \\
& V_{E_{y}^{*} E_{y}^{*}}^{(2)}=V_{H_{z}^{*} H_{z}^{*}}^{(2)}=\left(\begin{array}{ccc}
-i \omega \tilde{\psi}(\omega)^{-1}+\sigma & c_{\psi 0} & 0 \\
0 & -i \omega\left(1-\frac{r_{\psi 0}^{2}}{\omega^{2}}\right) & r_{\psi 0}^{2} \\
0 & 0 & -i \omega
\end{array}\right)
\end{aligned}
$$


This determinant can be simplified to the following explicit expression

$$
\begin{aligned}
\operatorname{det} \mathrm{V} & =(-1)^{5}\left(\omega^{2}-\omega_{x 0}^{2}\right)\left(\omega^{2}-\omega_{y 0}^{2}\right)\left(\omega^{2}-\omega_{\mu 0}^{2}\right)\left(\omega^{2}-r_{\psi 0}^{2}\right)^{2} \\
& \times \operatorname{det}\left(\begin{array}{ccccc}
-i \omega \tilde{\varepsilon}_{x}(\omega) & 0 & -i k_{y} & 0 & 0 \\
0 & -i \omega \tilde{\varepsilon}_{y}(\omega) & i k_{x} & \sigma & 0 \\
-i k_{y} & i k_{x} & -i \omega \tilde{\mu}(\omega) & 0 & \sigma \\
0 & 0 & i k_{x} & -i \omega \tilde{\psi}(\omega)^{-1}+\sigma & 0 \\
0 & i k_{x} & 0 & 0 & -i \omega \tilde{\psi}(\omega)^{-1}+\sigma
\end{array}\right) .
\end{aligned}
$$

Substracting from the second row the 4 th row multiplied by $\sigma\left(-i \omega \tilde{\psi}^{-1}+\sigma\right)^{-1}$, and from the third row the fifth row multiplied by $\sigma\left(-i \omega \tilde{\psi}^{-1}+\sigma\right)^{-1}$, we obtain

$$
\begin{aligned}
\operatorname{det} \mathrm{V} & =(-1)^{5}\left(\omega^{2}-\omega_{x 0}^{2}\right)\left(\omega^{2}-\omega_{y 0}^{2}\right)\left(\omega^{2}-\omega_{\mu 0}^{2}\right)\left(\omega^{2}-r_{\psi 0}^{2}\right)^{2} \\
& \times \operatorname{det}\left(\begin{array}{ccccc}
-i \omega \tilde{\varepsilon}_{x}(\omega) & 0 & -i k_{y} & 0 & 0 \\
0 & -i \omega \tilde{\varepsilon}_{y}(\omega) & i k_{x}\left(1-\frac{\sigma}{-i \omega \tilde{\psi}^{-1}+\sigma}\right) & 0 & 0 \\
-i k_{y} & i k_{x}\left(1-\frac{\sigma}{-i \omega \tilde{\psi}^{-1}+\sigma}\right) & -i \omega \tilde{\mu}(\omega) & 0 & 0 \\
0 & 0 & i k_{x} & -i \omega \tilde{\psi}(\omega)^{-1}+\sigma & 0 \\
0 & i k_{x} & 0 & 0 & -i \omega \tilde{\psi}(\omega)^{-1}+\sigma
\end{array}\right) .
\end{aligned}
$$

From this, immediately,

$$
\begin{aligned}
\operatorname{det} \mathrm{V} & =(-1)^{5}\left(\omega^{2}-\omega_{x 0}^{2}\right)\left(\omega^{2}-\omega_{y 0}^{2}\right)\left(\omega^{2}-\omega_{\mu 0}^{2}\right)\left(\omega^{2}-r_{\psi 0}^{2}\right)^{2}\left(-i \omega \tilde{\psi}^{-1}+\sigma\right)^{2} \\
& \times \operatorname{det}\left(\begin{array}{ccc}
-i \omega \tilde{\varepsilon}_{x}(\omega) & 0 & -i k_{y} \\
0 & -i \omega \tilde{\varepsilon}_{y}(\omega) & i k_{x}\left(1-\frac{\sigma}{-i \omega \tilde{\psi}^{-1}+\sigma}\right) \\
-i k_{y} & i k_{x}\left(1-\frac{\sigma}{-i \omega \tilde{\psi}^{-1}+\sigma}\right) & -i \omega \tilde{\mu}(\omega)
\end{array}\right) \\
& =-i \omega \tilde{\varepsilon}_{x}^{-1} \tilde{\varepsilon}_{y}^{-1}(-1)^{5}\left(\omega^{2}-\omega_{x 0}^{2}\right)\left(\omega^{2}-\omega_{y 0}^{2}\right)\left(\omega^{2}-\omega_{\mu 0}^{2}\right)\left(\omega^{2}-r_{\psi 0}^{2}\right)^{2}\left(-i \omega \tilde{\psi}^{-1}+\sigma\right)^{2}\left(\omega^{2} \tilde{\mu}-\tilde{\varepsilon}_{y}^{-1} k_{x}^{2}\left(1-\frac{\sigma \tilde{\psi}}{i \omega}\right)^{-2}-\tilde{\varepsilon}_{x}^{-1} k_{y}^{2}\right)
\end{aligned}
$$




\section{Appendix G. Construction of a Function $\psi(s)$ Satisfying Conditions of THEOREM 5.12}

In [9], the authors have proposed a procedure to construct the function $\psi(s)$ that results in stable perfectly matched layer for isotropic dispersive systems, based on a result similar to Theorem 5.12. Let us briefly comment on the extension (and a minor modification) of their procedure to anisotropic dispersive systems in question. Consider a passive sesquilinear form $A(u, v)$ that satisfies Assumption 5.1. Let us describe a procedure of choosing a function $\psi(s)$ for the PML change of variables that satisfies conditions of Theorem 5.12.

The condition (NSC1) of Theorem 5.12 can be rewritten alternatively in the following form. Either the sign of $\tilde{\psi}$ is constant on the real axis (and thus is positive, since $\tilde{\psi}(\omega) \rightarrow 1$ as $\omega \rightarrow \infty$ ), or there exists a set of intervals $\mathcal{I}_{k}=\left(\omega_{2 k-1}, \omega_{2 k}\right), k=1, \ldots, m$, with $0 \leq \omega_{1}<\omega_{2} \leq \omega_{3}<\ldots \leq \omega_{2 k-1}<\omega_{2 m}=+\infty$, s.t. the sign of the function $\tilde{\psi}(\omega)$ on an interval $\mathcal{I}_{k}$ is opposite to the sign of $\tilde{\psi}(\omega)$ on the intervals $\mathcal{I}_{k-1}$ and $\mathcal{I}_{k+1}$. These signs are defined by the signs of $\tilde{a}(\omega)$. Let us remark that $\omega_{2 m}=+\infty$, since as $\omega \rightarrow+\infty, \tilde{c}(\omega) \tilde{a}(\omega)>0$, and thus, $\tilde{\psi}(\omega)>0$.

The case when the sign of $\tilde{\psi}$ is constant on $\mathbb{R}$, is trivially reduced to using the Bérenger's PML $\tilde{\psi}=1$. Otherwise, following [9], we consider two cases:

(1) $m=2 \ell-1, \ell>1$. In this case the sign of $\tilde{\psi}(\omega)$ on $\mathcal{I}_{1}$ coincides with the sign of $\tilde{\psi}(\omega)$ on $\left(\omega_{2 m},+\infty\right)$, i.e. is positive. One can verify that the following holds true:

$$
\begin{aligned}
\omega \in\left(\omega_{4 k-3}, \omega_{4 k-2}\right) & \Longrightarrow \tilde{\psi}(\omega) \geq 0, \\
\omega \in\left(\omega_{4 k-1}, \omega_{4 k}\right) & \Longrightarrow \tilde{\psi}(\omega) \leq 0 .
\end{aligned}
$$

Thus, one of the choices of $\tilde{\psi}(\omega)$ is given by

$$
\tilde{\psi}(\omega)=\prod_{k=1}^{\ell-1} \frac{\left(\omega^{2}-r_{k}^{2}\right)}{\left(\omega^{2}-p_{k}^{2}\right)}, \quad r_{k} \in\left[\omega_{4 k-2}, \omega_{4 k-1}\right], p_{k} \in\left[\omega_{4 k}, \omega_{4 k+1}\right] .
$$

First of all, notice that for all $k=1, \ldots, \ell-2$, the following interlacing property holds true: $r_{k} \leq \omega_{4 k-1}<$ $\omega_{4 k} \leq p_{k} \leq \omega_{4 k+1}<\omega_{4 k+2} \leq r_{k+1}$. Applying Lemma 4.10, we see that $\tilde{\psi}(\omega)$ satisfies $\operatorname{Re}(\bar{s} \psi(s))>0$ in $\mathbb{C}_{+}$. It remains only to verify that $\tilde{\psi}$ defined as above satisfies the sign conditions (108). Taking $\omega_{*} \in\left(\omega_{4 j-3}, \omega_{4 j-2}\right)$, we can split

$$
\tilde{\psi}(\omega)=\prod_{k=1}^{j-1} \frac{\left(\omega^{2}-r_{k}^{2}\right)}{\left(\omega^{2}-p_{k}^{2}\right)} \prod_{k=j}^{\ell-2} \frac{\left(\omega^{2}-r_{k}^{2}\right)}{\left(\omega^{2}-p_{k}^{2}\right)}=\tilde{\psi}_{j}^{(1)}(\omega) \tilde{\psi}_{j}^{(2)}(\omega) .
$$

One sees that all the poles and zeros of $\tilde{\psi}_{j}^{(1)}(\omega)$ are smaller than $\omega_{*}$ (notice that $p_{j-1} \leq \omega_{4 j-3}$ ), hence $\tilde{\psi}_{j}^{(1)}\left(\omega_{*}\right)>0$. Similarly, all the poles and zeros of $\tilde{\psi}_{j}^{(2)}(\omega)$ are larger than $\omega_{*}$ (since $r_{j} \geq \omega_{4 j-2}$ ), hence by direct computation $\tilde{\psi}_{j}^{(2)}\left(\omega_{*}\right)>0$. Therefore, $\tilde{\psi}\left(\omega_{*}\right)>0$.

Similarly one can verify the validity of $(108)$ for $\omega_{*} \in\left(\omega_{4 j-1}, \omega_{4 j}\right)$.

(2) $m=2 \ell, \ell>1$. In this case the sign of $\tilde{\psi}(\omega)$ on $\mathcal{I}_{1}$ is negative. One can verify that the following holds true:

$$
\begin{aligned}
\omega \in\left(\omega_{4 k-3}, \omega_{4 k-2}\right) & \Longrightarrow \tilde{\psi}(\omega) \leq 0, \\
\omega \in\left(\omega_{4 k-1}, \omega_{4 k}\right) & \Longrightarrow \tilde{\psi}(\omega) \geq 0 .
\end{aligned}
$$


One of the choices of $\tilde{\psi}(\omega)$ is provided by

$$
\tilde{\psi}(\omega)=\prod_{k=1}^{\ell-1} \frac{\left(\omega^{2}-r_{k}^{2}\right)}{\left(\omega^{2}-p_{k}^{2}\right)}, \quad r_{1} \in\left[0, \omega_{1}\right], r_{k} \in\left[\omega_{4 k}, \omega_{4 k+1}\right], p_{1} \in\left[\omega_{2}, \omega_{3}\right], p_{k} \in\left[\omega_{4 k+2}, \omega_{4 k+3}\right]
$$

As before, the condition $\operatorname{Re}(\bar{s} \psi(s))>0$ for $s \in \mathbb{C}_{+}$, and the sign conditions (109) can be ensured by the direct use of Lemma 4.10 and computations as in the previous case.

\section{REFERENCES}

[1] J.-P. Berenger, A perfectly matched layer for the absorption of electromagnetic waves, J. Comput. Phys. 114 (2) (1994) $185-200$.

[2] J.-P. Berenger, Three-dimensional perfectly matched layer for the absorption of electromagnetic waves, J. Comput. Phys. 127 (2) (1996) 363-379.

[3] J. Jacquot, Description non lineáeire auto-cohérente de la propagation d'ondes radiofréquences et de la périphérie d'un plasma magnétisé, Ph.D. thesis, Université de Lorraine (2013).

[4] J. Jacquot, L. Colas, F. Clairet, M. Goniche, S. Heuraux, J. Hillairet, G. Lombard, D. Milanesio, 2d and 3d modeling of wave propagation in cold magnetized plasma near the tore supra icrh antenna relying on the perfecly matched layer technique, Plasma Physics and Controlled Fusion 55 (11) (2013) 115004.

[5] M. Chevalier, T. Chevalier, U. Inan, A PML utilizing k-vector information as applied to the whistler mode in a magnetized plasma, Antennas and Propagation, IEEE Transactions on 54 (8) (2006) 2424-2429.

[6] É. Bécache, P. Joly, M. Kachanovska, V. Vinoles, Perfectly matched layers in negative index metamaterials and plasmas, in: CANUM 2014-42e Congrès National d'Analyse Numérique, Vol. 50 of ESAIM Proc. Surveys, EDP Sci., Les Ulis, 2015, pp. $113-132$.

[7] E. Bécache, P. Joly, M. Kachanovska, Stable Perfectly Matched Layers for a Cold Plasma in a Strong Background Magnetic Field In preparation.

[8] S. A. Cummer, Perfectly matched layer behavior in negative refractive index materials, IEEE Antennas and Wireless Propagation Letters 3 (1) (2004) 172-175.

[9] E. Bécache, P. Joly, V. Vinoles, On the analysis of perfectly matched layers for a class of dispersive media. Application to negative index metamaterials. Submitted. URL https://hal.archives-ouvertes.fr/hal-01327315

[10] E. Bécache, S. Fauqueux, P. Joly, Stability of perfectly matched layers, group velocities and anisotropic waves, J. Comput. Phys. 188 (2) (2003) 399-433.

[11] D. Appelö, T. Hagstrom, G. Kreiss, Perfectly matched layers for hyperbolic systems: general formulation, well-posedness, and stability, SIAM J. Appl. Math. 67 (1) (2006) 1-23.

[12] Toufic, P. Joly, J. Rodrguez, I. Terrasse, Coupling discontinuous galerkin methods and retarded potentials for transient wave propagation on unbounded domains, Journal of Computational Physics 230 (15) (2011) 5877 - 5907.

[13] L. Banjai, C. Lubich, F.-J. Sayas, Stable numerical coupling of exterior and interior problems for the wave equation, Numerische Mathematik 129 (4) (2015) 611-646.

[14] T. Hohage, F. Schmidt, L. Zschiedrich, Solving time-harmonic scattering problems based on the pole condition. I. Theory, SIAM J. Math. Anal. 35 (1) (2003) 183-210 (electronic).

[15] D. Ruprecht, A. Schädle, F. Schmidt, Transparent boundary conditions based on the pole condition for time-dependent, two-dimensional problems, Numer. Methods Partial Differential Equations 29 (4) (2013) 1367-1390.

[16] B. Engquist, A. Majda, Absorbing boundary conditions for the numerical simulation of waves, Math. Comp. 31 (139) (1977) 629-651.

[17] T. Hagstrom, T. Warburton, A new auxiliary variable formulation of high-order local radiation boundary conditions: corner compatibility conditions and extensions to first-order systems, Wave Motion 39 (4) (2004) 327-338, new computational methods for wave propagation.

[18] D. Givoli, B. Neta, High-order non-reflecting boundary scheme for time-dependent waves, J. Comput. Phys. 186 (1) (2003) 24-46.

[19] F. Collino, High order absorbing boundary conditions for wave propagation models: straight line boundary and corner cases, in: Second International Conference on Mathematical and Numerical Aspects of Wave Propagation (Newark, DE, 1993), SIAM, Philadelphia, PA, 1993, pp. 161-171.

[20] D. Baffet, T. Hagstrom, D. Givoli, Double absorbing boundary formulations for acoustics and elastodynamics, SIAM J. Sci. Comput. 36 (3) (2014) A1277-A1312.

[21] A. Welters, Y. Avniel, S. G. Johnson, Speed-of-light limitations in passive linear media, Phys. Rev. A 90 (2014) 023847. 
[22] L. Halpern, J. Rauch, Bérenger/Maxwell with discontinous absorptions: existence, perfection, and no loss, in: Séminaire Laurent Schwartz-Équations aux dérivées partielles et applications. Année 2012-2013, Sémin. Équ. Dériv. Partielles, École Polytech., Palaiseau, 2014, pp. Exp. No. X, 20.

[23] Z. Chen, Convergence of the time-domain perfectly matched layer method for acoustic scattering problems, Int. J. Numer. Anal. Model. 6 (1) (2009) 124-146.

[24] M. Cassier, P. Joly, M. Kachanovska, On properties of passive systems In preparation.

[25] M. Gustafsson, D. Sjöberg, Sum rules and physical bounds on passive metamaterials, New Journal of Physics 12 (4) (2010) 043046.

[26] O. Brune, Synthesis of a finite two-terminal network whose driving-point impedance is a prescribed function of frequency, Journal of Mathematics and Physics 10 (1-4) (1931) 191-236.

[27] P. I. Richards, A special class of functions with positive real part in a half-plane, Duke Math. J. 14 (1947) $777-786$.

[28] B. J. Levin, Distribution of zeros of entire functions, revised Edition, Vol. 5 of Translations of Mathematical Monographs, American Mathematical Society, Providence, R.I., 1980, translated from the Russian by R. P. Boas, J. M. Danskin, F. M. Goodspeed, J. Korevaar, A. L. Shields and H. P. Thielman.

[29] B. Simon, Orthogonal polynomials on the unit circle. Part 2, Vol. 54 of American Mathematical Society Colloquium Publications, American Mathematical Society, Providence, RI, 2005, spectral theory.

[30] R. Dautray, J.-L. Lions, Mathematical analysis and numerical methods for science and technology. Vol. 5, Springer-Verlag, Berlin, 1992, evolution problems. I, With the collaboration of Michel Artola, Michel Cessenat and Hélène Lanchon, Translated from the French by Alan Craig.

[31] H.-O. Kreiss, J. Lorenz, Initial-Boundary Value Problems and the Navier-Stokes Equations, Academic Press, Inc., 1989.

[32] C. Lubich, On the multistep time discretization of linear initial-boundary value problems and their boundary integral equations, Numerische Mathematik 67 (1994) 365-389.

[33] V. Domínguez, F.-J. Sayas, Some properties of layer potentials and boundary integral operators for the wave equation, J. Integral Equations Appl. 25 (2) (2013) 253-294.

[34] Sayas, Retarded Potentials and Time Domain Boundary Integral Equations. A Road Map, online.

[35] F. J. Sayas, Retarded potentials and time domain boundary integral equations: a road-map, lecture Notes. URL http://www.math.udel.edu/ fjsayas/TDBIEclassnotes2012.pdf

[36] N. Dunford, J. T. Schwartz, Linear operators. Part I, Wiley Classics Library, John Wiley \& Sons, Inc., New York, 1988, general theory, With the assistance of William G. Bade and Robert G. Bartle, Reprint of the 1958 original, A Wiley-Interscience Publication.

[37] A. R. Laliena, F.-J. Sayas, Theoretical aspects of the application of convolution quadrature to scattering of acoustic waves, Numer. Math. 112 (4) (2009) 637-678.

[38] C. Lubich, Convolution quadrature revisited, BIT 44 (3) (2004) 503-514.

[39] P. Joly, An elementary introduction to the construction and the analysis of perfectly matched layers for time domain wave propagation, SëMA J. (57) (2012) 5-48.

[40] J.-P. Bérenger, Perfectly Matched Layer (PML) for Computational Electromagnetics, Synthesis Lectures on Computational Electromagnetics 2 (1) (2007) 1-117.

[41] W. C. Chew, W. H. Weedon, A 3D perfectly matched medium from modified Maxwell's equations with stretched coordinates, Microwave and Optical Technology Letters 7 (13) (1994) 599-604.

[42] L. Zhao, A. C. Cangellaris, GT-PML: generalized theory of perfectly matched layers and its application to the reflectionless truncation of finite-difference time-domain grids, IEEE Transactions on Microwave Theory and Techniques 44 (12) (1996) 2555-2563.

[43] K. S. Yee, Numerical solution of initial boundary value problems involving maxwell's equations in isotropic media, IEEE Trans. Antennas Propag 14 (3) (1966) 302-307.

[44] J. Diaz, P. Joly, A time domain analysis of PML models in acoustics, Comput. Methods Appl. Mech. Engrg. 195 (29-32) (2006) 3820-3853.

[45] F. Q. Hu, A stable, perfectly matched layer for linearized Euler equations in unsplit physical variables, J. Comput. Phys. 173 (2) (2001) 455-480.

[46] E. Demaldent, S. Imperiale, Perfectly matched transmission problem with absorbing layers: application to anisotropic acoustics in convex polygonal domains, Internat. J. Numer. Methods Engrg. 96 (11) (2013) 689-711.

[47] M. Kachanovska, Stable perfectly matched layers for a class of anisotropic dispersive models. Part II: Energy Estimates and Stability in Corners. In preparation.

[48] C. Lubich, Convolution quadrature and discretized operational calculus. I, Numer. Math. 52 (2) (1988) $129-145$.

[49] C. Lubich, Convolution quadrature and discretized operational calculus. II, Numer. Math. 52 (4) (1988) $413-425$.

[50] C. Lubich, A. Ostermann, Runge-Kutta methods for parabolic equations and convolution quadrature, Math. Comp. 60 (201) (1993) 105-131.

[51] L. Banjai, S. Sauter, Rapid solution of the wave equation in unbounded domains, SIAM J. Numer. Anal. 47 (1) (2008/09) $227-249$. 
[52] L. Banjai, D. Peterseim, Parallel multistep methods for linear evolution problems, IMA J. Numer. Anal. 32 (3) (2012) 12171240.

[53] L. D. Landau, L. P. Pitaevskii, E. M. Lifshitz, Electrodynamics of continuous media, Vol. 8, Elsevier, 1984.

[54] V. G. Veselago, The Electrodynamics of Substances with Simultaneously Negative Values of $\epsilon$ and $\mu$, Soviet Physics Uspekhi 10 (1968) 509-514.

[55] É. Bécache, P. Joly, On the analysis of Bérenger's perfectly matched layers for Maxwell's equations , ESAIM: Mathematical Modelling and Numerical Analysis 36(1) (2002) 87-119.

[56] M. Cassier, G. Milton, Bounds on Stieltjes functions and their applications to fundamental limits of broadband passive cloaking in quasistatic regimeIn preparation.

[57] W. F. Donoghue, Jr., Monotone matrix functions and analytic continuation, Springer-Verlag, New York-Heidelberg, 1974, die Grundlehren der mathematischen Wissenschaften, Band 207.

[58] I. S. Gradshteyn, I. M. Ryzhik, Table of integrals, series, and products, eighth Edition, Elsevier/Academic Press, Amsterdam, 2015, Translated from the Russian, Translation edited and with a preface by Daniel Zwillinger and Victor Moll, Revised from the seventh edition.

[59] N. G. Čebotarev, N. N. Meĭman, The Routh-Hurwitz problem for polynomials and entire functions, Trudy Mat. Inst. Steklov. 26 (1949) 331, (in Russian).

[60] C. Carathéodory, Conformal Representation, Cambridge University Press, 1969. 